\title{
Factorization for groomed jet substructure beyond the next-to-leading logarithm
}

\author{
Christopher Frye, Andrew J. Larkoski, Matthew D. Schwartz and Kai Yan \\ Center for the Fundamental Laws of Nature, Harvard University, \\ 17 Oxford Street, Cambridge, MA 02138, U.S.A. \\ E-mail: frye@physics.harvard.edu, larkoski@physics.harvard.edu, \\ schwartz@physics.harvard.edu, kyan@physics.harvard.edu
}

ABSTRACT: Jet grooming algorithms are widely used in experimental analyses at hadron colliders to remove contaminating radiation from within jets. While the algorithms perform a great service to the experiments, their intricate algorithmic structure and multiple parameters has frustrated precision theoretic understanding. In this paper, we demonstrate that one particular groomer called soft drop actually makes precision jet substructure easier. In particular, we derive a factorization formula for a large class of soft drop jet substructure observables, including jet mass. The essential observation that allows for this factorization is that, without the soft wide-angle radiation groomed by soft drop, all singular contributions are collinear. The simplicity and universality of the collinear limit in QCD allows us to show that to all orders, the normalized differential cross section has no contributions from non-global logarithms. It is also independent of process, up to the relative fraction of quark and gluon jets. In fact, soft drop allows us to define this fraction precisely. The factorization theorem also explains why soft drop observables are less sensitive to hadronization than their ungroomed counterparts. Using the factorization theorem, we resum the soft drop jet mass to next-to-next-to-leading logarithmic accuracy. This requires calculating some clustering effects that are closely related to corresponding effects found in jet veto calculations. We match our resummed calculation to fixed order results for both $e^{+} e^{-} \rightarrow$ dijets and $p p \rightarrow Z+j$ events, producing the first jet substructure predictions (groomed or ungroomed) to this accuracy for the LHC.

Keywords: Jets, NLO Computations

ArXiv EPrint: 1603.09338 


\section{Contents}

1 Introduction 1

2 Observables 4

2.1 Soft drop grooming algorithm 4

2.2 Energy correlation functions 5

3 Factorization theorem $\quad 6$

3.1 Power counting and modes $\quad 6$

$\begin{array}{lll}3.2 & \text { Factorization and refactorization } & 8\end{array}$

$\begin{array}{ll}3.3 \text { The single scale of the collinear-soft function } & 10\end{array}$

4 Consequences of factorization theorem $\quad \mathbf{1 3}$

$\begin{array}{lll}4.1 & \text { Absence of non-global logarithms to all orders } & 13\end{array}$

$\begin{array}{ll}4.2 \text { Process independence } & 14\end{array}$

$\begin{array}{lll}4.3 & \text { Hadronization corrections } & 15\end{array}$

5 Achieving NNLL accuracy $\quad 16$

$\begin{array}{ll}5.1 & \text { NNLL for } \alpha=2, \beta=0\end{array}$

$\begin{array}{lll}5.1 .1 & \text { Two-loop soft function } & 18\end{array}$

5.1.2 Two-loop anomalous dimension and comparison with EVENT2 21

$\begin{array}{lll}5.2 & \text { Reclustering with anti- } k_{T} & 22\end{array}$

5.3 NNLL for $\alpha=2, \beta \geq 0 \quad 24$

6 Matching NNLL to fixed order in $e^{+} e^{-} \rightarrow$ dijets 26

$\begin{array}{lll}\text { 6.1 Matching resummation to fixed-order } & 27\end{array}$

6.2 Comparison to Monte Carlo 28

7 Matching NNLL to fixed order in $p p \rightarrow Z+j \quad 32$

7.1 Resummed cross section in $p p \rightarrow Z+j \quad 33$

$\begin{array}{lll}7.2 & \text { Matching resummation to fixed-order } & 35\end{array}$

$\begin{array}{lll}7.3 & \text { Comparison to Monte Carlo } & 38\end{array}$

8 Conclusions $\quad 40$

A Three-loop $\beta$-function and cusp anomalous dimension $\quad 42$

B Hard function $\quad 42$

B.1 $e^{+} e^{-} \rightarrow q \bar{q} \quad 43$

B.2 $e^{+} e^{-} \rightarrow g g \quad 43$

C The global soft function $\quad 44$ 
D Jet functions $\quad 45$

D.1 Quark jets 46

D.2 Gluon jets 46

$\begin{array}{ll}\text { E Collinear-soft function } & 47\end{array}$

$\begin{array}{lll}\text { F } & \text { Resummation } & 48\end{array}$

G Renormalization group evolution of $D_{k}$

\section{Introduction}

The high luminosity proton collisions at the Large Hadron Collider (LHC) enable an unprecedented sensitivity to rare and high scale physics. The cost of such high luminosities is the presence of significant amounts of pile-up radiation present in every event, arising from numerous secondary proton collisions per bunch crossing. Pile-up is truly uncorrelated with the hard scattering and can contaminate any potential measurement. This is particularly important for measurements made on jets, for which pile-up can effect a large systematic bias in observables like the jet mass. In searches for resonances that decay to boosted electroweak objects which have definite masses, pile-up can significantly degrade the ability to separate signal from background. Over the past several years, numerous methods [1-11] have been developed for grooming jets and events for pile-up mitigation and removal, and are now standard experimental tools at both ATLAS and CMS experiments. Especially in analyses of jets, measurements made at the LHC often involve some form of grooming.

With this motivation, it is imperative to understand these jet grooming techniques from first principles QCD. There have been a few studies of the theoretical aspects of jet groomers $[6,8,12,13]$, with predictions for jet-observable distributions calculated to nextto-leading logarithmic (NLL) accuracy for two widely used jet groomers: the modified mass drop tagger (mMDT) and soft drop. These explicit analytic studies showed that these jet groomers not only have desired experimental properties, but can also dramatically simplify theoretical calculations as compared to their ungroomed counterparts. Non-global logarithms (NGLs) that arise from correlations between in- and out-of-jet scales have proven to be a significant obstruction to resummation of ungroomed jet observables to NLL accuracy and beyond. In particular, it was demonstrated by explicit calculation in refs. [6, 8, 12] that mMDT and soft drop groomers eliminate the leading non-global logarithms in jet mass distributions [14]. mMDT and soft drop pave the way for systematically improvable resummed predictions of jet observables.

In this paper, we open the door to systematically improvable jet substructure calculations by presenting an all-orders factorization theorem for the soft-drop [8] groomed observables using soft-collinear effective theory (SCET) [15-18]. An overview of the method 


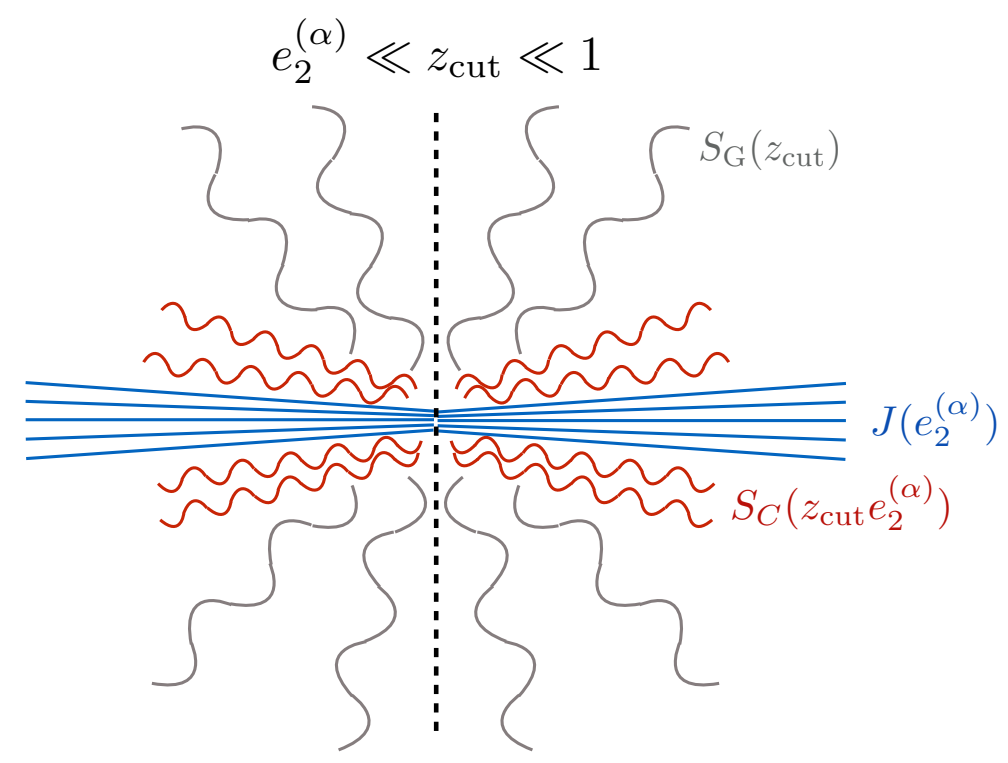

Figure 1. Schematic of the modes in the factorization theorem for soft-drop groomed hemispheres in $e^{+} e^{-} \rightarrow$ dijets events. $S_{\mathrm{G}}\left(z_{\text {cut }}\right)$ denotes the soft wide-angle modes, $S_{C}\left(z_{\text {cut }} e_{2}^{(\alpha)}\right)$ denotes the collinear-soft modes, and $J\left(e_{2}^{(\alpha)}\right)$ denotes the jet modes.

we discuss here and some of our results were presented recently in ref. [19]. This paper provides a more detailed presentation of those results as well as a derivation of the factorization formula and its remarkable properties.

The soft drop groomer walks through the branching history of a jet, discarding soft branches until a sufficiently hard branching is found. This is enforced by effectively requiring

$$
\frac{\min \left[E_{i}, E_{j}\right]}{E_{i}+E_{j}}>z_{\text {cut }}\left(\frac{\theta_{i j}}{R}\right)^{\beta}
$$

where $E_{i}$ and $E_{j}$ are the energies of the particles in that step of the branching, $\theta_{i j}$ is their relative angle, and $R$ is the radius of the jet. $z_{\text {cut }}$ is a parameter that sets the scale of soft, wide angle emissions in the jet; the typical value is $z_{\text {cut }}=0.1$. $\beta$ is a parameter that controls the aggressiveness of the groomer: $\beta=\infty$ removes the groomer, $\beta=0$ coincides with mMDT and is simply an energy cut, and $\beta<0$ removes all soft and collinear singularities. We will consider $\beta \geq 0$. If eq. (1.1) is not satisfied, the softer of the two branches is removed from the jet, and the grooming procedure continues on the harder branch. When eq. (1.1) is satisfied, the procedure terminates and the groomed jet is returned. For concreteness, on this groomed jet, we measure the two-point energy correlation functions $e_{2}^{(\alpha)}$ with angular exponent $\alpha>0$ [20-22].

In $e^{+} e^{-} \rightarrow$ dijets events, the factorization formula we derive in this paper for soft-drop groomed left and right hemisphere jets is:

$$
\frac{d^{2} \sigma}{d e_{2, L}^{(\alpha)} d e_{2, R}^{(\alpha)}}=H\left(Q^{2}\right) S_{\mathrm{G}}\left(z_{\text {cut }}\right)\left[S_{C}\left(z_{\text {cut }} e_{2, L}^{(\alpha)}\right) \otimes J\left(e_{2, L}^{(\alpha)}\right)\right]\left[S_{C}\left(z_{\text {cut }} e_{2, R}^{(\alpha)}\right) \otimes J\left(e_{2, R}^{(\alpha)}\right)\right]
$$


This factorization theorem applies when $z_{\text {cut }} \ll 1$ and the left- and right-hemisphere energy correlation functions are asymptotically small: $e_{2, L}^{(\alpha)}, e_{2, R}^{(\alpha)} \ll z_{\text {cut }} \ll 1$. We illustrate the physical configuration corresponding to this factorization theorem in figure 1. In eq. (1.2), $H\left(Q^{2}\right)$ is the hard function for $e^{+} e^{-} \rightarrow q \bar{q} . S_{\mathrm{G}}\left(z_{\text {cut }}\right)$ is the global soft function, which is only sensitive to the scale set by $z_{\text {cut }}$ since all of its emissions fail soft drop. $S_{C}\left(z_{\text {cut }} e_{2, L}^{(\alpha)}\right)$ is a soft function that is boosted along the direction of the jet in the left hemisphere; its corresponding modes are referred to as collinear-soft [23-28]. Emissions in $S_{C}\left(z_{\text {cut }} e_{2, L}^{(\alpha)}\right)$ may or may not pass the soft drop requirement and are therefore constrained by both $z_{\text {cut }}$ and $e_{2, L}^{(\alpha)}$. Importantly, this collinear-soft mode depends on only a single scale which we generically denote by $z_{\text {cut }} e_{2, L}^{(\alpha)}$. (For $\alpha \neq 2$ or $\beta>0$, the single scale is a different combination of $z_{\text {cut }}$ and $e_{2, L}^{(\alpha)}$; we simply call it $z_{\text {cut }} e_{2, L}^{(\alpha)}$ for notational brevity.) $J\left(e_{2, L}^{(\alpha)}\right)$ is the jet function for the left hemisphere jet, and all emissions in the jet function parametrically pass the soft drop requirement. Thus, the jet function is independent of the scale set by $z_{\text {cut }}$, and only depends on $e_{2, L}^{(\alpha)}$. $\otimes$ denotes convolution in $e_{2, L}^{(\alpha)}$, and a similar collinear-soft and jet factorization exists for the right hemisphere.

As we will explain in detail, there are several important consequences of this factorization formula. Because the formula depends on the observables $e_{2, L}^{(\alpha)}, e_{2, R}^{(\alpha)}$ only through collinear objects each of which has a single scale, there are no non-global logarithms. The elimination of the purely soft contribution also makes the shape of soft-drop groomed jet shapes largely independent of what else is going on in the event. For example, the shape of the left hemisphere jet mass is independent of what is present in the right hemisphere. Additionally, the scale associated with the collinear-soft mode is parametrically larger than the soft scale associated with ungroomed masses, so non-perturbative corrections such as hadronization are correspondingly smaller.

This factorization theorem allows us to go beyond NLL accuracy to arbitrary accuracy. In this paper, we show that next-to-next-to-leading logarithmic (NNLL) accuracy is readily achievable. We focus on $\alpha=2$ where the two-point energy correlation function is equal to the squared jet mass (up to a trivial normalization). This lets us extract most of the necessary two-loop anomalous dimensions from the existing literature. For $\beta=0$, the global soft function $S_{\mathrm{G}}\left(z_{\text {cut }}\right)$ is closely related to the soft function with an energy veto $[28,29]$ which is known to two-loop order. There are additional clustering effects from the soft drop algorithm, but these are straightforward to calculate. Interestingly, we find that the clustering effects in the soft drop groomer are intimately related to similar effects observed in jet veto calculations [30-34]. For $\beta=1$, we compute the two-loop anomalous dimension of $S_{\mathrm{G}}\left(z_{\text {cut }}\right)$ numerically using the fixed-order code EVENT2 [35]. ${ }^{1}$ We thereby achieve full NNLL resummation for the soft-drop groomed jet mass. ${ }^{2}$

\footnotetext{
${ }^{1}$ While we will not do it in this paper, one could use the results of ref. [36] which calculates the anomalous dimension of the soft function for event-wide (recoil-free) angularities [37-40] or energy correlation functions with arbitrary angular exponent. This would enable us to extend our results to the case with $\alpha \neq 2$.

${ }^{2}$ The jet mass has been calculated at NNLL using other methods [41-43] as has 2-subjettiness [44]. However, without grooming the jets, there are non-global logarithms which are not resummed (and which may or may not be quantitatively important) and uncontrollable sensitivity to pileup (which is very quantitatively important).
} 
It is straightforward to generalize from $e^{+} e^{-}$to $p p$ collisions, since the distribution is determined by collinear physics within the jet, independent of the initial state. The main new ingredient in $p p$ collisions is that jets may be initiated by quarks or gluons. As we will show, soft-drop grooming the jet enables an infrared and collinear safe definition of the jet flavor at leading power in $e_{2}^{(\alpha)}$ and $z_{\text {cut }}$ by simply summing the flavors of partons in the groomed jet. Using this procedure, we are able to match our NNLL resummed distribution of soft-drop groomed jet mass to fixed order results for $p p \rightarrow Z+j$ events (including relative $\mathcal{O}\left(\alpha_{s}^{2}\right)$ corrections to the Born process).

The outline of this paper is as follows. In section 2, we review the definition of the soft drop grooming algorithm and the energy correlation functions. In section 3, we present the factorization theorem for soft-drop groomed energy correlation functions in $e^{+} e^{-} \rightarrow$ dijets events. In this section, we will also present a detailed power-counting analysis of soft-dropped observables to determine the range of validity of the factorization theorem. Our factorization theorem has many non-trivial consequences, which we review in section 4. These include absence of non-global logarithms, process independence, and small hadronization corrections. In section 5 , we describe and present the ingredients necessary for NNLL resummation. Here, we also describe our method for extracting anomalous dimensions from EVENT2. We then match our NNLL results with fixed-order calculations for $e^{+} e^{-}$collisions in section 6 and for $p p \rightarrow Z+j$ events in section 7 , comparing with Monte Carlo simulations in each case. In section 8 , we summarize and conclude. The calculational details for NNLL resummation are collected in appendices.

\section{Observables}

In this section, we review the soft drop grooming algorithm and the energy correlation functions. Although previous work has focused on jets produced in $p p$ collisions, we will provide definitions for both lepton and hadron collider environments.

\subsection{Soft drop grooming algorithm}

Given a set of constituents of a jet with radius $R$, the soft drop grooming algorithm [8] proceeds in the following way:

1. Recluster the jet with a sequential $k_{T}$-type [45-47] jet algorithm. This produces an infrared and collinear (IRC) safe branching history of the jet. The $k_{T}$ clustering metric for jets in $e^{+} e^{-}$collisions is

$$
d_{i j}^{e^{+} e^{-}}=\min \left[E_{i}^{2 p}, E_{j}^{2 p}\right]\left(1-\cos \theta_{i j}\right)
$$

where $E_{i}, E_{j}$ are the energies of particles $i$ and $j$ and $\theta_{i j}$ is their relative angle. $p$ is a real number that defines the particular jet algorithm. For jets produced in $p p$ collisions, the $k_{T}$ clustering metric is

$$
d_{i j}^{p p}=\min \left[p_{T i}^{2 p}, p_{T j}^{2 p}\right] R_{i j}^{2}
$$


where $p_{T i}, p_{T j}$ are the transverse momenta of particles $i$ and $j$ with respect to the beam and $R_{i j}^{2}$ is their relative angle in the pseudorapidity-azimuth angle plane.

While the original implementation of soft drop was restricted to reclustering with the Cambridge/Aachen algorithm $(p=0)$ [48-50], we will also briefly consider reclustering with the anti- $k_{T}$ algorithm $(p=-1)$ [51] in section 5.2.

2. Sequentially step through the branching history of the reclustered jet. At each branching, check the soft drop criterion. For $e^{+} e^{-}$collisions, we require

$$
\frac{\min \left[E_{i}, E_{j}\right]}{E_{i}+E_{j}}>z_{\text {cut }}\left(\sqrt{2} \frac{\sin \frac{\theta_{i j}}{2}}{\sin \frac{R}{2}}\right)^{\beta} .
$$

This is known as the soft drop criterion. If the branching fails this requirement, then the softer of the two daughter branches is removed from the jet. The soft drop groomer then continues to the next branching in the remaining clustering history. For $p p$ collisions, the soft drop criterion is

$$
\frac{\min \left[p_{T i}, p_{T j}\right]}{p_{T i}+p_{T j}}>z_{\text {cut }}\left(\frac{R_{i j}}{R}\right)^{\beta} .
$$

3. The procedure continues until the soft drop criterion is satisfied. At that point, soft drop terminates, and returns the jet groomed of the branches that failed the soft drop criterion.

Once the jet has been groomed, any observable can be measured on its remaining constituents.

\subsection{Energy correlation functions}

On jets that have been groomed by soft drop, we measure the two-point energy correlation functions [20-22]. We do this mainly for concreteness; the general properties of the factorized formula we will present apply for a much broader class of observables. For jets in $e^{+} e^{-}$collisions, the two-point energy correlation function $e_{2}^{(\alpha)}$ is

$$
\left.e_{2}^{(\alpha)}\right|_{e^{+} e^{-}}=\frac{1}{E_{J}^{2}} \sum_{i<j \in J} E_{i} E_{j}\left(\frac{2 p_{i} \cdot p_{j}}{E_{i} E_{j}}\right)^{\alpha / 2},
$$

where $E_{J}$ is the sum of the energies of particles in the jet, the sum runs over distinct pairs $i, j$ of particles in the jet, $p_{i}$ is the four-vector momentum of particle $i$, and the angular exponent $\alpha$ is required to be greater than 0 for IRC safety. For $\alpha=2$ and a jet that has massless constituents, the two-point energy correlation function reduces to the normalized, squared jet mass:

$$
\left.e_{2}^{(2)}\right|_{e^{+} e^{-}}=\frac{m_{J}^{2}}{E_{J}^{2}} .
$$

The energy correlation functions have the nice property that they are insensitive to recoil effects $[20,40]$ and do not include explicit axes in their definition. 
For jets produced in $p p$ collisions, the energy correlation functions are appropriately modified by replacing spherical coordinates with cylindrical coordinates:

$$
\left.e_{2}^{(\alpha)}\right|_{p p}=\frac{1}{p_{T J}^{2}} \sum_{i<j \in J} p_{T i} p_{T j} R_{i j}^{\alpha},
$$

where $p_{T J}$ is the transverse momentum of the jet and $R_{i j}$ is the separation of particles $i$ and $j$ in the pseudorapidity-azimuthal angle plane. For jets at central rapidities and in the limit that all particles in the jet are collinear, eq. (2.7) reduces to eq. (2.5). This property in particular will enable us to recycle results calculated in $e^{+} e^{-}$collisions to the case of $p p$ collisions.

\section{Factorization theorem}

In this section, we derive the factorization formula for energy correlation functions measured on soft-drop groomed jets in the region of phase space where $e_{2}^{(\alpha)} \ll z_{\text {cut }} \ll 1$ using SCET [15-18]. We begin with a power counting analysis based on the scales $e_{2}^{(\alpha)}$ and $z_{\text {cut }}$ relevant to soft-drop groomed jets. This enables us to identify all modes and their momentum scalings that contribute at leading power. Using these scales and the associated modes, we derive the factorization formula. We then show that the jet function in the factorization formula can be re-factorized due to a collinear-soft mode which decouples from the collinear-but-not-soft modes as a result of soft drop.

\subsection{Power counting and modes}

For jets on which the soft drop groomer is applied and the energy correlation functions are measured, there are three relevant dimensonless scales: the jet radius $R$, the soft drop parameter $z_{\text {cut }}$, and $e_{2}^{(\alpha)}$. Typically, jet radii are $R \sim 1$. We are interested in the singular region $e_{2}^{(\alpha)} \rightarrow 0$ for a fixed value of $z_{\text {cut }}$. Thus we can assume $e_{2}^{(\alpha)} \ll z_{\text {cut }}$. We will also assume $z_{\text {cut }} \ll 1$ to refactorize the jet function. The limits $R \ll 1$ or $z_{\text {cut }} \sim 1$ could be considered as well, but are beyond the scope of our analysis.

We will use scaling arguments to identify the regions of phase space that are present at leading power and then take the limit where each region becomes a separate sector, that no longer interacts with the other regions.

For a jet to have $e_{2}^{(\alpha)} \ll 1$, all particles must be either soft or collinear to the jet axis. In particular, a particle with energy $E=z E_{J}$ at an angle $\theta$ from the jet axis must satisfy

$$
z \theta^{\alpha} \lesssim e_{2}^{(\alpha)}
$$

This is a line in the $\log (1 / z)-\log (1 / \theta)$ plane, as shown in figure 2. Anything below the dashed line in this figure is too hard to be consistent with a given value of $e_{2}^{(\alpha)}$. The soft drop criterion is that

$$
z_{\text {cut }} \lesssim z \theta^{-\beta}
$$

This is the region below the solid line in figure 2 . 


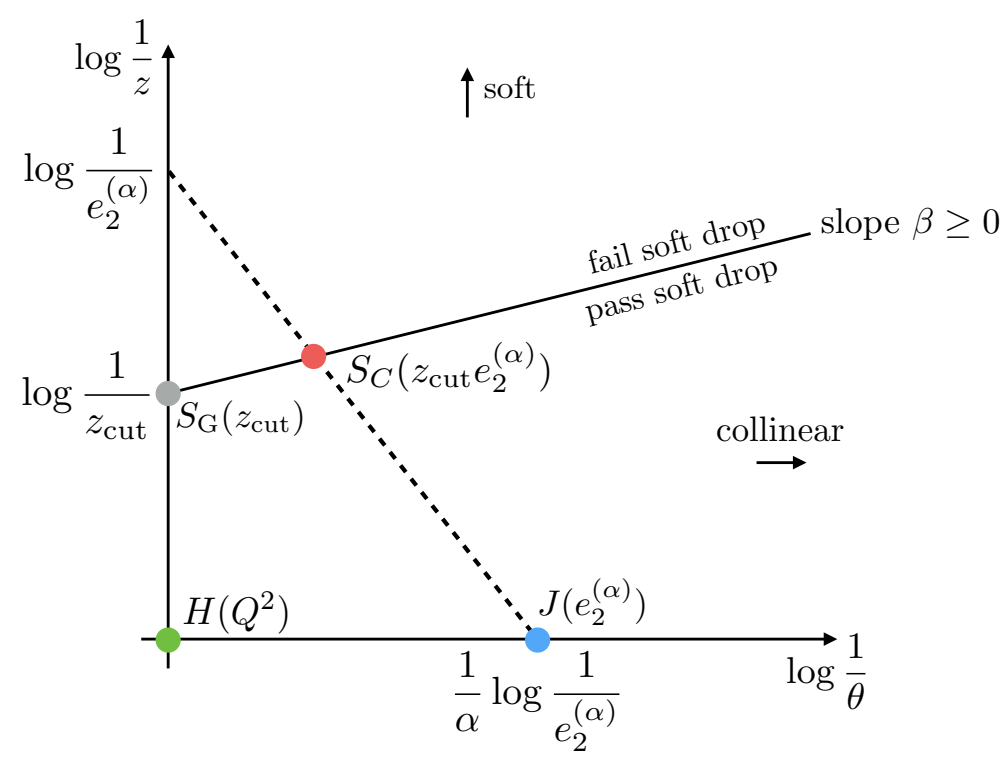

Figure 2. Location of modes appearing in the soft drop factorization theorem in the plane defined by energy fraction $z$ and splitting angle $\theta$ of emissions in the jet. The solid diagonal line separates the regions of phase space where emissions pass and fail soft drop. All emissions along the dashed line that pass soft drop contribute at leading power to the measured value of $e_{2}^{(\alpha)}$.

To find the relevant modes for the factorized expression, we need to identify the distinct characteristic momentum scalings that approach the singular regions of phase space in the limit $e_{2}^{(\alpha)} \ll z_{\text {cut }} \ll 1$. For a particular scaling, the constraints in eqs. (3.1) and (3.2) will either remain relevant or decouple. We can characterize the relevant regions by their scalings in light-cone coordinates. Defining $n^{\mu}$ as the jet direction and $\bar{n}^{\mu}$ as the direction backwards to the jet, then light-cone coordinates are triplets $p=\left(p^{-}, p^{+}, p_{\perp}\right)$ where $p^{-}=$ $\bar{n} \cdot p, p^{+}=n \cdot p$ and $p_{\perp}$ are the components transverse to $n$. On-shell massless particles have $p^{+} p^{-}=p_{\perp}^{2}$. The energy fraction is $z=p^{0} / Q=\frac{1}{2}\left(p^{+}+p^{-}\right) / Q$ and the angle to the jet axis in the collinear limit is $\theta=p_{\perp} / p^{0}$.

We start with the soft modes, emitted at large angles $\theta \sim 1$, but still within the jet. If such radiation were to pass soft drop, with energy fraction greater than $z_{\text {cut }}$, it would set $e_{2}^{(\alpha)} \gtrsim z_{\text {cut }}$; this contradicts our assumed hierarchy $e_{2}^{(\alpha)} \ll z_{\text {cut }}$. Therefore, soft wide-angle radiation is removed by soft drop and is not constrained by $e_{2}^{(\alpha)}$. These modes thus have momenta that scale like

$$
p_{s} \sim z_{\text {cut }} Q(1,1,1) .
$$

They contribute only to the normalization of the distribution, not to its shape.

Next consider the collinear radiation, emitted at small angles $\theta \ll 1$. All collinear radiation has $p^{-} \gg p^{+}$. Then, from eq. (3.1), we find

$$
e_{2}^{(\alpha)} \sim \frac{\left(p^{+}\right)^{\alpha / 2}\left(p^{-}\right)^{1-\alpha / 2}}{Q}
$$

Collinear modes can either have $z \sim 1$ or be parameterically soft $z \ll 1$. 


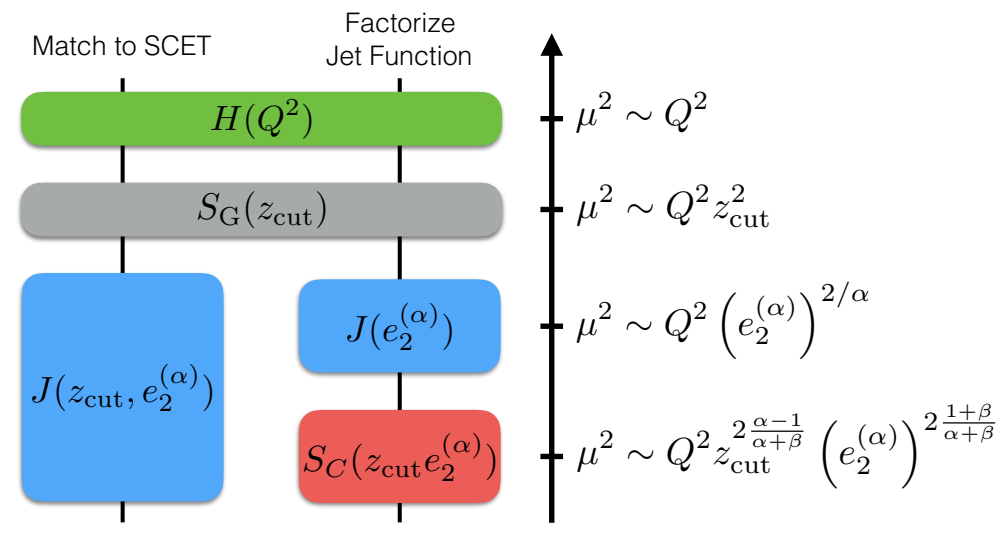

Figure 3. Illustration of the multi-stage matching procedure to derive the soft drop factorization theorem. As discussed in the text, we first match QCD to SCET, then factorize the jet function into collinear and collinear-soft modes. Canonical scales of all modes in the factorization theorem are shown on the right, ordered in virtuality where we assume that $\alpha>1$ and $\beta \geq 0$.

For modes with $z \sim 1$, we have $z \gg z_{\text {cut }}$. Thus $p^{-} \sim Q$ and $p^{+} \sim Q\left(e_{2}^{(\alpha)}\right)^{2 / \alpha}$ independent of $z_{\text {cut }}$. Their scaling is

$$
p_{c} \sim Q\left(1,\left(e_{2}^{(\alpha)}\right)^{2 / \alpha},\left(e_{2}^{(\alpha)}\right)^{1 / \alpha}\right) .
$$

We call these modes collinear modes, although strictly they are not-soft collinear modes.

Collinear radiation that can have $z \sim z_{\text {cut }} \ll 1$ we call collinear-soft. In this case, $p^{-} \sim z Q$ and $p^{+} \sim \theta^{2} z Q$. These modes are simultaneously compatible with eqs. (3.1) and (3.2). Their scaling is determined by saturating these parametric relationships, which leads to

$$
p_{\text {cs }} \sim\left(z_{\text {cut }}\right)^{\frac{\alpha}{\alpha+\beta}}\left(e_{2}^{(\alpha)}\right)^{\frac{\beta}{\alpha+\beta}} Q\left(1,\left(\frac{e_{2}^{(\alpha)}}{z_{\text {cut }}}\right)^{\frac{2}{\alpha+\beta}},\left(\frac{e_{2}^{(\alpha)}}{z_{\text {cut }}}\right)^{\frac{1}{\alpha+\beta}}\right) .
$$

This is the point in phase space labeled $S_{C}\left(z_{\text {cut }} e_{2}^{(\alpha)}\right)$ in figure 2 .

\subsection{Factorization and refactorization}

With the relevant scalings identified, we proceed to derive the factorization formula. For simplicity, we focus on the case of $e^{+} e^{-} \rightarrow$ hemisphere jets, with $e_{2}^{(\alpha)}$ measured on each hemisphere. Jets at hadron colliders can be treated similarly, as we discuss in section 4 . Figure 3 illustrates the relevant modes and their scales.

We begin with the usual SCET factorization formula, in the absence of soft drop grooming. The hard, collinear and soft modes are separated in the limit of small observables. This leads to $[39,52,53]$

$$
\frac{d^{2} \sigma}{d e_{2, L}^{(\alpha)} d e_{2, R}^{(\alpha)}}=H\left(Q^{2}\right) \times S\left(e_{2, L}^{(\alpha)}, e_{2, R}^{(\alpha)}\right) \otimes J\left(e_{2, L}^{(\alpha)}\right) \otimes J\left(e_{2, R}^{(\alpha)}\right)
$$

for the ungroomed hemispheres in $e^{+} e^{-} \rightarrow$ dijets events, provided $e_{2, L}^{(\alpha)}, e_{2, R}^{(\alpha)} \ll 1$. Here, $\otimes$ denotes convolution in $e_{2, L}^{(\alpha)}$ or $e_{2, R}^{(\alpha)}$ appropriately. To get to this equation, one can match to 
full QCD to get the hard function, then decouple the soft and collinear degrees of freedom to pull the jet and soft functions apart [15-18]. Alternatively, one can use the method of regions approach [54,55], or the on-shell phase space approach [56-58]. Importantly, $e_{2}^{(\alpha)}$ is insensitive to recoil effects from soft emissions that displace the jet axis from the direction of hard, collinear particles [20, 40], and so the jet and soft functions are completely decoupled.

Next we write down the hard-soft-jet factorization formula in the presence of soft drop grooming, assuming the hierarchy $e_{2}^{(\alpha)} \ll z_{\text {cut }} \ll 1$. With this assumption, soft radiation emitted at large angles must necessarily fail the soft drop criterion. Thus, all wide angle soft radiation in the jets (in this case, the hemisphere jets) is groomed and cannot contribute to the observable. All that remains of the global soft function is a

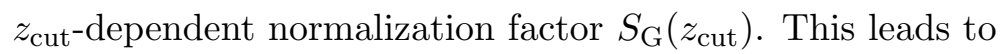

$$
\frac{d^{2} \sigma}{d e_{2, L}^{(\alpha)} d e_{2, R}^{(\alpha)}}=H\left(Q^{2}\right) \times S_{\mathrm{G}}\left(z_{\text {cut }}\right) \times J_{z e}\left(z_{\text {cut }}, e_{2, L}^{(\alpha)}\right) \times J_{z e}\left(z_{\text {cut }}, e_{2, R}^{(\alpha)}\right) .
$$

$S_{\mathrm{G}}\left(z_{\text {cut }}\right)$ gives the cross section for the radiation from a set of Wilson lines that fails the soft drop criterion. An explicit calculation of $S_{\mathrm{G}}$ for hemisphere jets at one-loop is given in appendix C. With the collinear and soft modes decoupled, we can lower the virtuality of the collinear modes without further matching.

The jet function $J_{z e}$ still depends on multiple scales, so to resum all the large logarithms it must be re-factorized. To see that it refactorizes, note first that in addition to being collinear, radiation in the jet function that is sensitive to the scale set by $z_{\text {cut }}$ must also be soft, by the assumption that $z_{\text {cut }} \ll 1$. Equivalently, emissions with order- 1 energy fractions are not constrained by the scale $z_{\text {cut }}$. We can thus factorize the jet function into two pieces depending on their energy fraction:

$$
J_{z e}\left(z_{\text {cut }}, e_{2}^{(\alpha)}\right)=J\left(e_{2}^{(\alpha)}\right) \otimes S_{C}\left(z_{\text {cut }} e_{2}^{(\alpha)}\right) .
$$

Here, $J\left(e_{2}^{(\alpha)}\right)$ is the jet function that only depends on $e_{2}^{(\alpha)}$ and only receives contributions from emissions with order-1 energy fraction. $S_{C}\left(z_{\text {cut }} e_{2}^{(\alpha)}\right)$ is the soft limit of the unfactorized jet function $J_{z e}\left(z_{\text {cut }}, e_{2}^{(\alpha)}\right)$. The scaling of the collinear and collinear-soft modes are given in eqs. (3.5) and (3.6) as discussed above. Note that, importantly, because the collinear-soft mode arises from refactorization of a jet function, it is a color singlet and only depends on two back-to-back directions. Because the jet function only depends on $e_{2}^{(\alpha)}$, it is sensitive to a single infrared scale.

The step in eq. (3.9) is the most unusual and important in the derivation. That the collinear-soft function depends on only a single combination of $z_{\text {cut }}$ and $e_{2}^{(\alpha)}$ is absolutely critical to being able to resum all the logs of $e_{2}^{(\alpha)}$. We therefore devote section 3.3 to showing explicitly that the collinear-soft function depends on a unique combination of $z_{\text {cut }}$ and $e_{2}^{(\alpha)}$ as determined by the parametric scaling of the modes of eq. (3.6), and so is also only sensitive to a single infrared scale.

Inserting eq. (3.9) into eq. (3.8) results in the factorization formula for soft drop energy correlation functions:

$$
\frac{d^{2} \sigma}{d e_{2, L}^{(\alpha)} d e_{2, R}^{(\alpha)}}=H\left(Q^{2}\right) S_{\mathrm{G}}\left(z_{\text {cut }}\right)\left[S_{C}\left(z_{\text {cut }} e_{2, L}^{(\alpha)}\right) \otimes J\left(e_{2, L}^{(\alpha)}\right)\right]\left[S_{C}\left(z_{\text {cut }} e_{2, R}^{(\alpha)}\right) \otimes J\left(e_{2, R}^{(\alpha)}\right)\right]
$$


The pieces of the factorization theorem are:

- $H\left(Q^{2}\right)$ is the hard function for production of an $e^{+} e^{-} \rightarrow$ dijets event.

- $S_{\mathrm{G}}\left(z_{\text {cut }}\right)$ is the global soft function. It integrates the radiation coming from Wilson lines in the jet directions that fails the soft drop criterion. Its modes fail soft drop and have momenta that scale as determined in eq. (3.3).

- $J\left(e_{2}^{(\alpha)}\right)$ is the jet function describing the emission of collinear radiation from a jet. Its modes parametrically pass soft drop and have momenta that scale as determined in eq. (3.5).

- $S_{C}\left(z_{\text {cut }} e_{2}^{(\alpha)}\right)$ is the collinear-soft function describing the emission of soft radiation boosted along the direction of a jet. Its modes may or may not pass soft drop and have momenta that scale as determined in eq. (3.6). We denote the single scale that the collinear-soft function depends on as $z_{\text {cut }} e_{2, L}^{(\alpha)}$ for brevity; it is shorthand for eq. (3.27).

We present the operator definitions and explicit one-loop results for all of these functions in the appendices.

The appearance of collinear-soft modes in this factorization theorem has some similarities and differences with respect to the identification of other collinear-soft modes in the literature [23-28]. The original construction of a collinear-soft mode in ref. [23] followed from boosting two jets in an event far from their center-of-mass frame in an effective theory of collinear dijets called $\mathrm{SCET}_{+}$. The collinear-soft mode in $\mathrm{SCET}_{+}$is sensitive to three Wilson line directions: the two from the collinear jets and the backward direction from boosting all other jets in the event. This collinear-soft mode was also exploited in ref. [26] in the resummation of jet observables that are sensitive to multi-prong substructure.

The collinear-soft mode in the factorization theorem presented here, however, is more similar to modes identified from the measurement of multiple observables on a jet, each of which is only sensitive to radiation about a single hard core [24-28]. For example, ref. [24] presented a factorization theorem for jets on which two angularities [37-40] are measured. At leading power, angularities are only sensitive to the hard jet core, and so the collinear-soft modes only know about two Wilson line directions: the jet axis and the backward direction. More recently, collinear-soft modes of this type have been used to resum NGLs [25, 27] and logarithms of the jet radius [28].

\subsection{The single scale of the collinear-soft function}

To demonstrate explicity that the collinear-soft function only depends on a single scale, we can make the following scaling argument. The collinear-soft function has the following form:

$$
S_{C}\left(z_{\mathrm{cut}}, e_{2}^{(\alpha)}\right)=\sum_{n} \mu^{2 n \epsilon} \int d \Pi_{n}\left|\mathcal{M}_{n}\right|^{2} \Theta_{\mathrm{SD}} \delta_{e_{2}^{(\alpha)}}
$$

Here, $n$ is the number of final state collinear-soft particles, $d \Pi_{n}$ is on-shell Lorentz-invariant phase space in $d=4-2 \epsilon$ dimensions:

$$
d \Pi_{n}=\prod_{i=1}^{n} \frac{d^{d} k_{i}}{(2 \pi)^{d}} 2 \pi \delta\left(k_{i}^{2}\right) \Theta\left(k_{i}^{0}\right),
$$


$\mu$ is the renormalization scale, and $\mathcal{M}_{n}$ is the amplitude for the production of the final state. $\Theta_{\mathrm{SD}}$ represents the soft drop grooming algorithm, which applies constraints on the final state and $\delta_{e_{2}^{(\alpha)}}$ represents the measurement of $e_{2}^{(\alpha)}$ on the final state:

$$
\delta_{e_{2}^{(\alpha)}}=\delta\left(e_{2}^{(\alpha)}-\frac{2^{\alpha}}{Q} \sum_{i}\left(k_{i}^{-}\right)^{1-\alpha / 2}\left(k_{i}^{+}\right)^{\alpha / 2}\right),
$$

where the sum runs over the set of final state particles $\{i\}$ that remain in the jet after grooming. To write this expression, we have used the definition of $e_{2}^{(\alpha)}$ from section 2.2 and expanded in the collinear-soft limit, as in eq. (3.4).

Now, we rescale the momenta in light-cone coordinates that appear in the phase space integral in the following way:

$$
\begin{aligned}
& k^{-} \rightarrow\left(z_{\text {cut }}\right)^{\frac{\alpha}{\alpha+\beta}}\left(e_{2}^{(\alpha)}\right)^{\frac{\beta}{\alpha+\beta}} k^{-}, \\
& k^{+} \rightarrow\left(z_{\text {cut }}\right)^{\frac{\alpha-2}{\alpha+\beta}}\left(e_{2}^{(\alpha)}\right)^{\frac{2+\beta}{\alpha+\beta}} k^{+}, \\
& k_{\perp} \rightarrow\left(z_{\text {cut }}\right)^{\frac{\alpha-1}{\alpha+\beta}}\left(e_{2}^{(\alpha)}\right)^{\frac{1+\beta}{\alpha+\beta}} k_{\perp} .
\end{aligned}
$$

At leading power in exactly $d=4$, the phase space measure $d \Pi_{n}$ and the squared matrix element $\left|\mathcal{M}_{n}\right|^{2}$ scale exactly inversely. Therefore, in $d$ dimensions, under this rescaling, we have

$$
d \Pi_{n}\left|\mathcal{M}_{n}\right|^{2} \rightarrow\left(\left(z_{\text {cut }}\right)^{\frac{\alpha-1}{\alpha+\beta}}\left(e_{2}^{(\alpha)}\right)^{\frac{1+\beta}{\alpha+\beta}}\right)^{-2 n \epsilon} d \Pi_{n}\left|\mathcal{M}_{n}\right|^{2} .
$$

Next, look at how the measurement functions $\Theta_{\mathrm{SD}}$ and $\delta_{e_{2}^{(\alpha)}}$ change under the rescaling of eq. (3.14). First, consider the soft drop groomer $\Theta_{\mathrm{SD}}$. This consists of two parts: one, the reclustering with the Cambridge/Aachen algorithm and the second, the energy requirement on the clustered particles. The clustering metric of the Cambridge/Aachen algorithm is just the pairwise angle

$$
d_{i j}^{\mathrm{C} / \mathrm{A}}=\theta_{i j}^{2},
$$

and a pair $\{i, j\}$ of particles in the jet are clustered if they have the smallest $d_{i j}^{\mathrm{C} / \mathrm{A}}$. Importantly, the reclustering of the jet with soft drop is completely inclusive: all particles in the jet are clustered with no jet radius parameter. Therefore, for collinear-soft modes, there are only three types of clustering constraints that can be enforced, depending on what

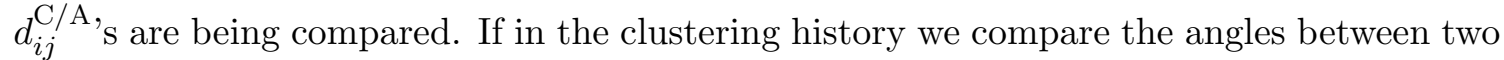
collinear-soft particles $i$ and $j$ to the jet axis, this corresponds to the constraint

$$
\Theta\left(\frac{k_{i}^{+}}{k_{i}^{-}}-\frac{k_{j}^{+}}{k_{j}^{-}}\right) .
$$

This is invariant under the rescalings of eq. (3.14). If in the clustering history we compare the angle between a collinear-soft particle $i$ to the jet axis and the angle between two collinear-soft particles $j$ and $k$, we have the constraint

$$
\Theta\left(\frac{k_{i}^{+}}{k_{i}^{-}}-\frac{k_{j} \cdot k_{k}}{k_{j}^{-} k_{k}^{-}}\right)
$$


which is also invariant under the rescalings of eq. (3.14). Finally, we can compare the angle between a pair of collinear-soft particles $i$ and $j$ to the angle between another pair of collinear-soft functions $k$ and $l$, this leads to

$$
\Theta\left(\frac{k_{i} \cdot k_{j}}{k_{i}^{-} k_{j}^{-}}-\frac{k_{k} \cdot k_{l}}{k_{k}^{-} k_{l}^{-}}\right)
$$

This too is invariant under eq. (3.14). Therefore, for all possible clustering structures, the Cambridge/Aachen algorithm is invariant under the rescalings of eq. (3.14).

The soft drop energy requirement on any number of particles that have been reclustered takes the form:

$$
\Theta\left(\sum_{i} z_{i}-z_{\text {cut }} \theta^{\beta}\right)
$$

where $z_{i}$ is the energy fraction of particle $i$ and $\theta$ is the angle that the cluster of particles $\{i\}$ makes with the jet axis. In terms of light-cone coordinates, this can be written as:

$$
\Theta\left(\sum_{i} z_{i}-z_{\mathrm{cut}} \theta^{\beta}\right)=\Theta\left(k^{-}-z_{\mathrm{cut}} Q\left(\frac{k_{\perp}}{k^{-}}\right)^{\beta}\right)
$$

where

$$
\begin{aligned}
& k_{\perp}=\left|\sum_{i} \vec{k}_{\perp, i}\right|, \\
& k^{-}=\sum_{i} k_{i}^{-} .
\end{aligned}
$$

Applying the rescalings of eq. (3.14), this constraint becomes

$$
\Theta\left(\sum_{i} z_{i}-z_{\mathrm{cut}} \theta^{\beta}\right) \rightarrow \Theta\left(\sum_{i} z_{i}-\theta^{\beta}\right)
$$

Note that the low scale $z_{\text {cut }}$ has been removed from this constraint.

Under the rescaling, the measurement constraint $\delta_{e_{2}^{(\alpha)}}$ becomes

$$
\delta\left(e_{2}^{(\alpha)}-\frac{2^{\alpha}}{Q} \sum_{i}\left(k_{i}^{-}\right)^{1-\alpha / 2}\left(k_{i}^{+}\right)^{\alpha / 2}\right) \rightarrow \frac{1}{e_{2}^{(\alpha)}} \delta\left(1-\frac{2^{\alpha}}{Q} \sum_{i}\left(k_{i}^{-}\right)^{1-\alpha / 2}\left(k_{i}^{+}\right)^{\alpha / 2}\right) .
$$

Therefore, the low scale $e_{2}^{(\alpha)}$ has been removed from this constraint.

Putting this all together, the collinear-soft function can be rewritten as

$$
S_{C}\left(z_{\text {cut }}, e_{2}^{(\alpha)}\right)=\sum_{n} \mu^{2 n \epsilon}\left(\left(z_{\text {cut }}\right)^{\frac{\alpha-1}{\alpha+\beta}}\left(e_{2}^{(\alpha)}\right)^{\frac{1+\beta}{\alpha+\beta}}\right)^{-2 n \epsilon} \frac{1}{e_{2}^{(\alpha)}} \int d \Pi_{n}\left|\mathcal{M}_{n}\right|^{2} \Theta_{\mathrm{SD}}^{z_{\mathrm{cut}}=1} \delta_{e_{2}^{(\alpha)}=1} .
$$

We have used the notation that $\Theta_{\mathrm{SD}}^{z_{\text {cut }}=1}$ is the soft drop grooming algorithm with $z_{\text {cut }}=1$ and $\delta_{e_{2}^{(\alpha)}=1}$ is the measurement with $e_{2}^{(\alpha)}=1$. All low scales have been explicitly removed 
from the phase space integral. This function is now seen to be a function only of the single scale

$$
\text { " } z_{\text {cut }} e_{2}^{(\alpha) "}=\left(z_{\text {cut }}\right)^{\frac{\alpha-1}{\alpha+\beta}}\left(e_{2}^{(\alpha)}\right)^{\frac{1+\beta}{\alpha+\beta}} .
$$

The quotes just mean that the left-hand side is our abbreviation for the unwieldly quantity on the right-hand side.

This proves that, to all orders, the collinear-soft function has dependence on only a single infrared scale, defined by this combination of $z_{\text {cut }}$ and $e_{2}^{(\alpha)}$. Notice that the proof relied on the choice of Cambridge/Aachen reclustering in the soft drop grooming algorithm.

This completes the derivation of factorization and re-factorization for soft-drop groomed hemispheres in $e^{+} e^{-}$collisions, on which two-point energy correlation functions have been measured. All functions in the factorized cross section in eq. (3.10) are sensitive to a single infrared scale and so all large logarithms can be resummed with the renormalization group.

\section{Consequences of factorization theorem}

Before we use the factorization theorem of eq. (3.10) to make predictions for the cross section, we discuss consequences of this formula in some detail. Because the factorization theorem was derived without respect to any fixed order, these results hold to all orders.

Many of these consequences follow from the fact that soft wide angle radiation does not contribute to the shape of the soft-drop groomed $e_{2}^{(\alpha)}$ distribution for $e_{2}^{(\alpha)} \ll z_{\text {cut }} \ll 1$, a property that persists even for jets at hadron colliders. For example, it follows immediately from this fact that the shape of such a distribution is insensitive to contamination from pile-up and underlying event.

In this section, we will furthermore prove that at leading power, there are no NGLs that affect the shape of the soft-drop groomed $e_{2}^{(\alpha)}$ distribution in this regime. This was explicitly shown at $\mathcal{O}\left(\alpha_{s}^{2}\right)$ in refs. [6, 8, 12] and plausibility arguments were presented for all orders, but this is the first proof. The factorization theorem also exhibits sample independence to a large degree, because the shape of the distribution is only sensitive to collinear physics. We will also demonstrate that soft-drop groomed energy correlation functions are less sensitive to hadronization than their ungroomed counterparts.

\subsection{Absence of non-global logarithms to all orders}

NGLs in cross sections of observables measured on individual hemispheres in $e^{+} e^{-}$collisions arise from a parametric separation of the scales in the hemispheres. Their leading effects are exclusively non-Abelian and quantify the correlation between the two hemispheres. Clearly, for a correlation to be present, there must be correlated radiation emitted into both hemispheres. If we measure the energy correlation functions $e_{2}^{(\alpha)}$ on both hemispheres and demand that $e_{2}^{(\alpha)} \ll 1$, then the radiation in the event must be soft wide-angle or collinear. At leading power, it is not possible to have correlations between different collinear directions (beyond total momentum conservation) as this would violate the collinear factorization of gauge theory amplitudes. Therefore, correlations and NGLs can only arise from soft, wideangle radiation in the event with these assumptions. 
The factorization theorem for ungroomed hemisphere energy correlation functions is $[39,52,53]$

$$
\left.\frac{d^{2} \sigma}{d e_{2, L}^{(\alpha)} d e_{2, R}^{(\alpha)}}\right|_{\text {ug }}=H\left(Q^{2}\right) S\left(e_{2, L}^{(\alpha)}, e_{2, R}^{(\alpha)}\right) \otimes J\left(e_{2, L}^{(\alpha)}\right) \otimes J\left(e_{2, R}^{(\alpha)}\right),
$$

where "ug" denotes ungroomed. The cross section explicitly depends on soft wide-angle radiation through the soft function $S\left(e_{2, L}^{(\alpha)}, e_{2, R}^{(\alpha)}\right)$, and so if either $e_{2, L}^{(\alpha)} \ll e_{2, R}^{(\alpha)}$ or $e_{2, R}^{(\alpha)} \ll e_{2, L}^{(\alpha)}$, NGLs will be present in this factorization theorem. Because the soft function depends on two scales, all of the singular dependence cannot be determined by renormalization group invariance. More generally, non-global structure present in the soft function has been studied at $\mathcal{O}\left(\alpha_{s}^{2}\right)$ [59-61] and beyond [62,63] and recently, methods have been developed to control all-orders behavior [25, 27, 64, 65]. However, NGLs represent an obstruction to resummation of the cross section to NLL and beyond.

For groomed hemisphere energy correlation functions, our factorization theorem instead takes the form of eq. (3.10):

$$
\frac{d^{2} \sigma}{d e_{2, L}^{(\alpha)} d e_{2, R}^{(\alpha)}}=H\left(Q^{2}\right) S_{\mathrm{G}}\left(z_{\text {cut }}\right)\left[S_{C}\left(z_{\text {cut }} e_{2, L}^{(\alpha)}\right) \otimes J\left(e_{2, L}^{(\alpha)}\right)\right]\left[S_{C}\left(z_{\text {cut }} e_{2, R}^{(\alpha)}\right) \otimes J\left(e_{2, R}^{(\alpha)}\right)\right]
$$

All soft, wide angle radiation throughout the event is described by $S_{\mathrm{G}}\left(z_{\text {cut }}\right)$, which is sensitive only to the single scale $z_{\text {cut }}$. Therefore, there are no NGLs present in this factorization theorem. Even with a hierarchy between $e_{2, L}^{(\alpha)}$ and $e_{2, R}^{(\alpha)}$, these observables are completely decoupled at leading power in $e_{2}^{(\alpha)}$ and $z_{\text {cut }}$. Additionally, the shape of the distribution is also independent of jet radius effects and the precise way in which the hemispheres are defined.

When we discuss soft-drop groomed jets in $p p$ collisions in section 7 , we will place no constraint on global soft radiation throughout the event, unlike the case of $e^{+} e^{-} \rightarrow$ hemisphere jets. Nevertheless, the shape of the soft-drop groomed $e_{2}^{(\alpha)}$ distribution will still have no NGLs, jet radius effects, etc., due to universality of the collinear limit of QCD amplitudes. The normalization, however, will in general be sensitive to scales both in the jet (set by $z_{\text {cut }}$ and the jet radius $R$ ) and scales outside of the jet (set by the partonic collision energy). To eliminate these effects in $p p$ collisions, we can normalize the cross section, say, to integrate to unity.

\subsection{Process independence}

Strictly speaking, the factorization theorem of eq. (3.10) depends on the process. It includes the hard function, which is process dependent, and a soft function, that knows about all hard jet directions. Nevertheless, there is a sense in which the factorization theorem is process independent. Normalizing the cross section completely removes the hard and soft function dependence. Then, by the universal collinear factorization of QCD amplitudes, if we are completely inclusive over the right hemisphere, then the differential cross section of the soft-drop groomed energy correlation function in the left hemisphere is given by

$$
\frac{d \sigma}{d e_{2, L}^{(\alpha)}}=\mathcal{N} S_{C}\left(z_{\text {cut }} e_{2, L}^{(\alpha)}\right) \otimes J\left(e_{2, L}^{(\alpha)}\right)
$$


where we assume that $e_{2, L}^{(\alpha)} \ll z_{\text {cut }} \ll 1$ and $\mathcal{N}$ is some normalization factor. That is, in the deep infrared where $e_{2, L}^{(\alpha)} \ll z_{\text {cut }} \ll 1$, all radiation in the groomed jet is constrained to be collinear. Therefore, in this limit and for a fixed jet energy, the shape of the distribution for quark jets is independent of the process that created the quark jets, due to the universality of QCD matrix elements in the collinear limit.

This collinear factorization property of soft-drop groomed observables can be exploited for jets in $p p$ collisions. Unlike the dominant case in $e^{+} e^{-}$collisions, jets at a $p p$ collider can be either quark or gluon. Of course, on a jet-by-jet level, we cannot determine whether a jet was initiated by a quark or gluon. However, for a given process, we can determine the relative fraction of quark and gluon jets in the sample. For jets produced at a $p p$ collider, the process independence manifests itself in the cross section as

$$
\frac{d \sigma^{p p}}{d e_{2}^{(\alpha)}}=D_{q} S_{C, q}\left(z_{\text {cut }} e_{2}^{(\alpha)}\right) \otimes J_{q}\left(e_{2}^{(\alpha)}\right)+D_{g} S_{C, g}\left(z_{\text {cut }} e_{2}^{(\alpha)}\right) \otimes J_{g}\left(e_{2}^{(\alpha)}\right),
$$

where $D_{q}\left(D_{g}\right)$ is proportional to the fraction of quark (gluon) jets in the sample. The relative fraction of quark and gluon jets can be determined from fixed-order calculations, using a simple algorithm for determining the flavor of a groomed jet. We will describe this in detail in section 7 when we match our resummed distribution to fixed order in $p p \rightarrow Z+j$ events.

\subsection{Hadronization corrections}

With a factorization formula, one can estimate the size and importance of non-perturbative corrections to the cross section. We will only consider non-perturbative corrections to the shape, as the normalization can be set by hand. Therefore, non-perturbative corrections can only enter into our factorization theorem via the jet or collinear-soft functions.

From eqs. (3.5) and (3.6), the scales appearing in the jet and collinear-soft functions are

$$
\begin{aligned}
\mu_{J} & =Q\left(e_{2}^{(\alpha)}\right)^{1 / \alpha} \\
\mu_{S_{C}} & =Q z_{\mathrm{cut}}^{\frac{\alpha-1}{\alpha+\beta}}\left(e_{2}^{(\alpha)}\right)^{\frac{1+\beta}{\alpha+\beta}} .
\end{aligned}
$$

If either of the scales approaches $\Lambda_{\mathrm{QCD}}$, then we expect there to be large corrections to the perturbative cross section due to non-perturbative physics. We can estimate when non-perturbative corrections become large by setting these scales to be $\Lambda_{\mathrm{QCD}}$. For $\alpha>1$ and $\beta \geq 0$, the collinear-soft mode has a lower virtuality than the collinear mode, so it will probe the non-perturbative region of phase space first. The value of $e_{2}^{(\alpha)}$ at which the collinear-soft mode becomes non-perturbative is

$$
\mu_{S_{C}}=\left.\Lambda_{\mathrm{QCD}} \Rightarrow e_{2}^{(\alpha)}\right|_{\mathrm{NP}} \simeq\left(\frac{\Lambda_{\mathrm{QCD}}}{z_{\mathrm{cut}} Q}\right)^{\frac{\alpha-1}{1+\beta}} \cdot \frac{\Lambda_{\mathrm{QCD}}}{Q} .
$$

This estimate can be compared with the Monte Carlo analysis of hadronization corrections to the soft-drop groomed energy correlation functions from ref. [8]. In particular, the estimate of eq. (4.7) of when non-perturbative corrections become important for $\alpha=2$ as a function of $\beta$ agrees exceptionally well with figure 10(a) of ref. [8]. 


\begin{tabular}{|c|cccccc|}
\hline & $\Gamma_{\text {cusp }}$ & $\gamma$ & $\beta$ & $\tilde{F}\left(\partial_{\omega}\right)$ & $c_{F}$ & Matching \\
\hline LL & $\alpha_{s}$ & - & $\alpha_{s}$ & - & - & - \\
NLL & $\alpha_{s}^{2}$ & $\alpha_{s}$ & $\alpha_{s}^{2}$ & $\alpha_{s}$ & - & $\alpha_{s}$ \\
NNLL & $\alpha_{s}^{3}$ & $\alpha_{s}^{2}$ & $\alpha_{s}^{3}$ & $\alpha_{s}^{2}$ & $\alpha_{s}$ & $\alpha_{s}^{2}$ \\
\hline
\end{tabular}

Table 1. $\alpha_{s}$-order of ingredients needed for resummation to the accuracy given. $\Gamma_{\text {cusp }}$ is the cusp anomalous dimension, $\gamma$ is the non-cusp anomalous dimension, and $\beta$ is the QCD $\beta$-function. $\tilde{F}\left(\partial_{\omega}\right)$ are the logarithms in the low-scale matrix elements that have been Laplace transformed and $c_{F}$ are constants in the low-scale matrix elements. The final column shows the relative order to which the resummed cross section can be matched to fixed-order.

For $\beta<\infty$, the soft drop groomer reduces the effect of non-perturbative corrections with respect to the ungroomed observable. This can be simply seen from eq. (4.7), in which the prefactor

$$
\left(\frac{\Lambda_{\mathrm{QCD}}}{z_{\mathrm{cut}} Q}\right)^{\frac{\alpha-1}{1+\beta}}
$$

approaches unity as the grooming is removed $(\beta \rightarrow \infty)$. This factor is less than 1 for $\beta<\infty$, provided $\alpha>1$ and $\Lambda_{\mathrm{QCD}}<z_{\text {cut }} Q$. For high energy jets, this suppression can be substantial. For example, for $\alpha=2$ (corresponding to jet mass) and $\beta=0$ (corresponding to mMDT groomer) non-perturbative effects become important at

$$
\left.e_{2}^{(2)}\right|_{\mathrm{NP}} ^{\beta=0} \simeq \frac{\Lambda_{\mathrm{QCD}}^{2}}{z_{\mathrm{cut}} Q^{2}}
$$

This agrees with the estimate of the size of nonperturbative corrections for the mMDT groomer from ref. [6].

\section{$5 \quad$ Achieving NNLL accuracy}

In this section, we determine the anomalous dimensions necessary to resum the large logarithms of soft-dropped energy correlation functions through NNLL accuracy. The practical details of how one assembles these ingredients, in the framework of SCET, to construct a resummed cross section are given in appendix F. We will discuss matching to fixed-order and demonstrate our ability to make phenomenological predictions in subsequent sections.

Resummation in SCET is accomplished with renormalization group evolution. Solving the renormalization group equations to a given logarithmic accuracy requires anomalous dimensions to a particular fixed order. The anomalous dimensions of the functions in the factorization theorem must sum to zero, because the cross section is independent of the renormalization scale.

Recall that, for $e^{+} e^{-} \rightarrow$ hemisphere jets, the factorization theorem for soft-drop groomed energy correlation functions is

$$
\frac{d^{2} \sigma}{d e_{2, L}^{(\alpha)} d e_{2, R}^{(\alpha)}}=H\left(Q^{2}\right) S_{\mathrm{G}}\left(z_{\text {cut }}\right)\left[S_{C}\left(z_{\text {cut }} e_{2, L}^{(\alpha)}\right) \otimes J\left(e_{2, L}^{(\alpha)}\right)\right]\left[S_{C}\left(z_{\text {cut }} e_{2, R}^{(\alpha)}\right) \otimes J\left(e_{2, R}^{(\alpha)}\right)\right]
$$


Table 1 presents the order to which anomalous dimensions and constants of the functions in this factorization theorem must be computed for particular logarithmic accuracy (see, e.g., ref. [66]). The cusp anomalous dimension $\Gamma_{\text {cusp }}$ and the QCD $\beta$-function are known through three-loop order [67-72] and we present them in appendix A. The hard function $H\left(Q^{2}\right)$ for $e^{+} e^{-} \rightarrow q \bar{q}$ is known to high orders and its non-cusp anomalous dimension $\gamma_{H}$ is known at three-loop order [73, 74]; we present the relevant pieces in appendix B. For arbitrary angular exponents $\alpha$ and $\beta$, little else in the factorization theorem is known at sufficiently high accuracy to resum to NNLL.

The goal of this section is to fill in the rest of the table, to achieve full NNLL accuracy. We start in section 5.1 restricting to $\alpha=2$ (jet mass) and $\beta=0$ (mMDT groomer). For this case, all of the missing ingredients can be determined by recycling results from the literature, up to calculable clustering effects from the soft drop algorithm. In section 5.3, we consider $\alpha=2$ and $\beta \geq 0$ and demonstrate that one can extract unknown two-loop non-cusp anomalous dimensions with EVENT2. It is possible to extend our analysis to angular exponents for the energy correlation functions beyond $\alpha=2$, but we do not do it in this paper. ${ }^{3}$

\subsection{NNLL for $\alpha=2, \beta=0$}

We first consider angular exponents $\alpha=2$ and $\beta=0$. In this case, the soft drop requirement enforced at every branching reduces to an energy cut

$$
\min \left[E_{i}, E_{j}\right]>z_{\text {cut }}\left(E_{i}+E_{j}\right)
$$

On the soft-drop groomed jets we then measure

$$
e_{2}^{(2)}=\frac{m_{g}^{2}}{E_{g}^{2}},
$$

where the subscript $g$ denotes that the mass and energy are measured on the groomed jet. The jet functions in the factorization theorem are independent of the soft drop groomer, so we are able to use results from the literature for these. The inclusive jet function has been calculated to two loops [75-78] and the non-cusp anomalous dimension of the inclusive jet function is known to three loops [79, 80]. We present the relevant expressions in appendix D.

This leaves the soft function $S_{\mathrm{G}}\left(z_{\text {cut }}\right)$ and the collinear-soft function $S_{C}\left(z_{\text {cut }} e_{2}^{(2)}\right)$ to be determined. Their one-loop expressions are easily calculable, and we present the results in appendix $\mathrm{C}$ and appendix E. To determine their two-loop non-cusp anomalous dimensions, we exploit the renormalization group consistency of the factorization theorem. The sum of the anomalous dimensions must vanish at each order:

$$
0=\gamma_{H}+\gamma_{S}+2 \gamma_{J}+2 \gamma_{S_{C}},
$$

\footnotetext{
${ }^{3}$ The two-loop non-cusp anomalous dimension of the soft function for event-wide (recoil-free) angularities [37-40] as a function of the angular exponent has been calculated in ref. [36]. Recoil-free angularities and two-point energy correlation functions have identical anomalous dimensions [40] and could be used in the same way as the calculation for $\alpha=2$.
} 
where $\gamma_{F}$ denotes the anomalous dimension of function $F$ in the factorization theorem, and we have used the symmetry of the left and right hemispheres of the event. Therefore, only one unknown anomalous dimension remains, which we take to be $\gamma_{S}$.

\subsubsection{Two-loop soft function}

To calculate the two-loop non-cusp anomalous dimension $\gamma_{S}$ we need to calculate the soft function $S_{\mathrm{G}}\left(z_{\text {cut }}\right)$ with two real emissions. The two-loop expression for the soft function is

$$
\left.S_{\mathrm{G}}\left(z_{\mathrm{cut}}\right)\right|_{\alpha_{s}^{2}}=\int\left[d^{d} k_{1}\right]_{+}\left[d^{d} k_{2}\right]_{+}\left|\mathcal{M}\left(k_{1}, k_{2}\right)\right|^{2} \Theta_{\mathrm{SD}} .
$$

Here, $\left[d^{d} k_{1}\right]_{+}$is the positive-energy on-shell phase space measure in $d=4-2 \epsilon$ dimensions:

$$
\left[d^{d} k_{1}\right]_{+}=\frac{d^{d} k_{1}}{(2 \pi)^{d}} 2 \pi \delta\left(k_{1}^{2}\right) \Theta\left(k_{1}^{0}\right)
$$

and $\left|\mathcal{M}\left(k_{1}, k_{2}\right)\right|^{2}$ is the squared matrix element for two soft emissions from a $q \bar{q}$ dipole. The explicit expression for $\left|\mathcal{M}\left(k_{1}, k_{2}\right)\right|^{2}$ can be found in ref. [81]. $\Theta_{\mathrm{SD}}$ is the phase space constraint imposed by the soft drop groomer. Recall that, for consistency with the assumed hierarchy $e_{2}^{(\alpha)} \ll z_{\text {cut }}$, soft modes must fail soft drop.

If the particles in the hemispheres are reclustered using the Cambridge/Aachen algorithm, $\Theta_{\mathrm{SD}}$ can be written as

$$
\begin{aligned}
\Theta_{\mathrm{SD}}= & \Theta\left(-\eta_{1} \eta_{2}\right) \Theta\left(z_{\text {cut }} \frac{Q}{2}-k_{1}^{0}\right) \Theta\left(z_{\text {cut }} \frac{Q}{2}-k_{2}^{0}\right) \\
& +\Theta\left(\eta_{1} \eta_{2}\right)\left[\Theta\left(\theta_{1 J}-\theta_{12}\right) \Theta\left(\theta_{2 J}-\theta_{12}\right) \Theta\left(z_{\text {cut }} \frac{Q}{2}-k_{1}^{0}-k_{2}^{0}\right)\right. \\
& \left.\quad+\left[1-\Theta\left(\theta_{1 J}-\theta_{12}\right) \Theta\left(\theta_{2 J}-\theta_{12}\right)\right] \Theta\left(z_{\text {cut }} \frac{Q}{2}-k_{1}^{0}\right) \Theta\left(z_{\text {cut }} \frac{Q}{2}-k_{2}^{0}\right)\right] .
\end{aligned}
$$

The first line of eq. (5.7) corresponds to particles 1 and 2 lying in different hemispheres (opposite rapidity with respect to the $q \bar{q}$ dipole), and so each particle individually must fail soft drop. $Q$ is the center of mass energy and so $Q / 2$ is the energy in one hemisphere. The second and third lines correspond to the configuration where both particles lie in the same hemisphere. $\theta_{12}$ is the angle between the particles and $\theta_{i J}($ for $i=1,2)$ is the angle particle $i$ makes with that hemisphere's axis. If $\theta_{12}$ is less than both $\theta_{1 J}$ and $\theta_{2 J}$ then, according to the Cambridge/Aachen algorithm, the soft particles are clustered first. Therefore, the sum of the energies of particles 1 and 2 must fail soft drop. If instead one of the particles is closer to the jet axis, then they are clustered separately and must individually fail soft drop.

To proceed, we separate the squared matrix element into Abelian and non-Abelian pieces, according to their color coefficient. At this order, the squared matrix element takes the form

$$
\left|\mathcal{M}\left(k_{1}, k_{2}\right)\right|^{2}=\left|\mathcal{M}_{\mathrm{n}-\mathrm{A}}\left(k_{1}, k_{2}\right)\right|^{2}+\frac{1}{2 !}\left|\mathcal{M}\left(k_{1}\right)\right|^{2}\left|\mathcal{M}\left(k_{2}\right)\right|^{2},
$$

Here, "n-A" denotes the non-Abelian component of the squared matrix element, which includes the $C_{F} C_{A}$ and $C_{F} n_{f} T_{R}$ color channels. The Abelian contribution is just the symmetrized product of the one-loop result, with a color factor of $C_{F}^{2}$. We will consider these two pieces separately, starting with the non-Abelian term. 
Non-Abelian clustering effects. Note that except for the effects from Cambridge/Aachen clustering, soft drop is just imposing a soft energy veto on each hemisphere. The two-loop soft function with a soft energy veto was calculated in ref. [29]. That calculation showed that the two-loop Abelian piece (proportional to $C_{F}^{2}$ ) to the energy vetoed soft function satisfies non-Abelian exponentiation. The two-loop non-cusp anomalous dimension for a hemisphere energy vetoed soft function is then purely non-Abelian and was extracted in ref. [28]. The non-Abelian part of the soft function with an energy veto at two-loops is

$$
\left.S_{\text {veto }}\right|_{\mathrm{n}-\mathrm{A}, \alpha_{s}^{2}}=\int\left[d^{d} k_{1}\right]_{+}\left[d^{d} k_{2}\right]_{+}\left|\mathcal{M}_{\mathrm{n}-\mathrm{A}}\left(k_{1}, k_{2}\right)\right|^{2} \Theta_{\text {veto }} .
$$

The phase space cut $\Theta_{\text {veto }}$ is

$$
\Theta_{\text {veto }}=\Theta\left(\Lambda-k_{1}^{0}-k_{2}^{0}\right)
$$

where $\Lambda$ is the veto scale. We can then write the two-loop soft function for soft drop as

$$
\left.S_{\mathrm{G}}\left(z_{\text {cut }}\right)\right|_{\mathrm{n}-\mathrm{A}, \alpha_{s}^{2}}=\left.S_{\text {veto }}\right|_{\mathrm{n}-\mathrm{A}, \alpha_{s}^{2}}+\int\left[d^{d} k_{1}\right]_{+}\left[d^{d} k_{2}\right]_{+}\left|\mathcal{M}_{\mathrm{n}-\mathrm{A}}\left(k_{1}, k_{2}\right)\right|^{2}\left[\Theta_{\mathrm{SD}}-\Theta_{\text {veto }}\right],
$$

where the veto scale is set to $\Lambda=z_{\text {cut }} Q / 2$. The difference between the soft drop and energy veto phase space constraints is purely a clustering effect, given by

$$
\begin{aligned}
\Theta_{\mathrm{SD}}-\Theta_{\text {veto }}= & \left\{\Theta\left(\eta_{1} \eta_{2}\right)\left[1-\Theta\left(\theta_{1 J}-\theta_{12}\right) \Theta\left(\theta_{2 J}-\theta_{12}\right)\right]+\Theta\left(-\eta_{1} \eta_{2}\right)\right\} \\
& \times \Theta\left(z_{\text {cut }} \frac{Q}{2}-k_{1}^{0}\right) \Theta\left(z_{\text {cut }} \frac{Q}{2}-k_{2}^{0}\right) \Theta\left(k_{1}^{0}+k_{2}^{0}-z_{\text {cut }} \frac{Q}{2}\right) .
\end{aligned}
$$

Eq. (5.10) enables us to calculate much more simply the two-loop non-cusp anomalous dimension of the soft function. The anomalous dimension can then be written as

$$
\gamma_{S}=\gamma_{\mathrm{veto}}+\gamma_{\mathrm{C} / \mathrm{A}} .
$$

$\gamma_{\text {veto }}$ is the two-loop non-cusp anomalous dimension of $S_{\text {veto }}$ extracted in ref. [28]:

$$
\gamma_{\text {veto }}^{\alpha_{s}^{2}}=\left(\frac{\alpha_{s}}{4 \pi}\right)^{2} C_{F}\left[\left(\frac{1616}{27}-56 \zeta_{3}\right) C_{A}-\frac{448}{27} n_{f} T_{R}-\frac{2 \pi^{2}}{3} \beta_{0}\right],
$$

where $\beta_{0}$ is the one-loop $\beta$-function coefficient:

$$
\beta_{0}=\frac{11}{3} C_{A}-\frac{4}{3} n_{f} T_{R} .
$$

Then, we only need to determine the contribution to the anomalous dimension from residual Cambridge/Aachen clustering effects, $\gamma_{\mathrm{C} / \mathrm{A}}$.

The non-Abelian clustering effects are contained in

$$
\left.S_{\mathrm{G}}\left(z_{\text {cut }}\right)\right|_{\mathrm{n}-\mathrm{A}, \alpha_{s}^{2}} ^{\mathrm{C} / \mathrm{A}}=\int\left[d^{d} k_{1}\right]_{+}\left[d^{d} k_{2}\right]_{+}\left|\mathcal{M}_{\mathrm{n}-\mathrm{A}}\left(k_{1}, k_{2}\right)\right|^{2}\left[\Theta_{\mathrm{SD}}-\Theta_{\text {veto }}\right] .
$$

The squared non-Abelian matrix element does not have collinear singularities when the angle of the particles from the jet axis is strongly ordered. Therefore, in this integral 
there is only a collinear divergence when the two emissions become collinear to the jet axis in a non-strongly ordered way. The coefficient of this divergence is proportional to the correction to the two-loop anomalous dimension due to clustering effects in the nonAbelian color channel. The divergence can be extracted with the standard plus-function prescription and the correction to the anomalous dimension can be found. While we were unable to find an analytic expression, its approximate numerical value is ${ }^{4}$

$$
\left.S_{\mathrm{G}}\left(z_{\mathrm{cut}}\right)\right|_{\mathrm{n}-\mathrm{A}, \alpha_{s}^{2}} ^{\mathrm{C} / \mathrm{A}}=\left(\frac{\alpha_{s}}{4 \pi}\right)^{2} C_{F}\left[-9.31 C_{A}-14.04 n_{f} T_{R}\right]\left(\frac{4 \mu^{2}}{z_{\text {cut }}^{2} Q^{2}}\right)^{2 \epsilon} \frac{1}{4 \epsilon}+\mathcal{O}\left(\epsilon^{0}\right) .
$$

The contribution to the anomalous dimension is then

$$
\gamma_{\mathrm{C} / \mathrm{A}}^{\mathrm{n}-\mathrm{A} \alpha_{s}^{2}}=\left(\frac{\alpha_{s}}{4 \pi}\right)^{2} C_{F}\left[-9.31 C_{A}-14.04 n_{f} T_{R}\right]
$$

Abelian clustering effects. The Abelian contribution can be calculated similarly. However, unlike the non-Abelian contribution, the exponentiation of the one-loop result will describe at least some of the two-loop Abelian piece. If the square of the one-loop result does not account for all of the two-loop result, then non-Abelian exponentiation breaks down. This does not mean that exponentiation breaks down or that the cross section cannot be resummed, just that the anomalous dimension of the purely Abelian piece will need to be corrected at every logarithmic order. So, for the two-loop non-cusp anomalous dimension, we need to determine the part of the soft function that is not accounted for by non-Abelian exponentiation.

To do this, we start from the full expression for the Abelian term at two-loops:

$$
\left.S_{\mathrm{G}}\left(z_{\mathrm{cut}}\right)\right|_{\mathrm{A}, \alpha_{s}^{2}}=\frac{1}{2 !} \int\left[d^{d} k_{1}\right]_{+}\left[d^{d} k_{2}\right]_{+}\left|\mathcal{M}\left(k_{1}\right)\right|^{2}\left|\mathcal{M}\left(k_{2}\right)\right|^{2} \Theta_{\mathrm{SD}} .
$$

We then add and subtract the one-loop phase space constraints:

$$
\begin{aligned}
\left.S_{\mathrm{G}}\left(z_{\text {cut }}\right)\right|_{\mathrm{A}, \alpha_{s}^{2}} & \\
= & \frac{1}{2 !} \int\left[d^{d} k_{1}\right]_{+}\left[d^{d} k_{2}\right]_{+}\left|\mathcal{M}\left(k_{1}\right)\right|^{2}\left|\mathcal{M}\left(k_{2}\right)\right|^{2} \Theta\left(z_{\text {cut }} \frac{Q}{2}-k_{1}^{0}\right) \Theta\left(z_{\text {cut }} \frac{Q}{2}-k_{2}^{0}\right) \\
& +\frac{1}{2 !} \int\left[d^{d} k_{1}\right]_{+}\left[d^{d} k_{2}\right]_{+}\left|\mathcal{M}\left(k_{1}\right)\right|^{2}\left|\mathcal{M}\left(k_{2}\right)\right|^{2}\left[\Theta_{\mathrm{SD}}-\Theta\left(z_{\text {cut }} \frac{Q}{2}-k_{1}^{0}\right) \Theta\left(z_{\text {cut }} \frac{Q}{2}-k_{2}^{0}\right)\right] .
\end{aligned}
$$

The difference between the phase space constraints is a clustering effect, given by

$$
\begin{aligned}
\Theta_{\mathrm{SD}}- & \Theta\left(z_{\text {cut }} \frac{Q}{2}-k_{1}^{0}\right) \Theta\left(z_{\text {cut }} \frac{Q}{2}-k_{2}^{0}\right) \\
= & -\Theta\left(\eta_{1} \eta_{2}\right) \Theta\left(\theta_{1 J}-\theta_{12}\right) \Theta\left(\theta_{2 J}-\theta_{12}\right) \\
& \times \Theta\left(z_{\text {cut }} \frac{Q}{2}-k_{1}^{0}\right) \Theta\left(z_{\text {cut }} \frac{Q}{2}-k_{2}^{0}\right) \Theta\left(k_{1}^{0}+k_{2}^{0}-z_{\text {cut }} \frac{Q}{2}\right) .
\end{aligned}
$$

\footnotetext{
${ }^{4}$ This anomalous dimension does not seem to be a linear combination of the usual transcendental numbers appearing in other two-loop anomalous dimensions.
} 
As with the non-Abelian term, this phase space constraint completely removes all soft divergences and the strongly-ordered collinear limit. The remaining divergence can be isolated by standard plus-function techniques. For the two-loop Abelian Cambridge/Aachen clustering term, we find the numerical result

$$
\left.S_{\mathrm{G}}\left(z_{\text {cut }}\right)\right|_{\mathrm{A}, \alpha_{s}^{2}} ^{\mathrm{C} / \mathrm{A}}=\left(\frac{\alpha_{s}}{4 \pi}\right)^{2} 34.01 C_{F}^{2}\left(\frac{4 \mu^{2}}{z_{\text {cut }}^{2} Q^{2}}\right)^{2 \epsilon} \frac{1}{4 \epsilon}+\mathcal{O}\left(\epsilon^{0}\right),
$$

for the second integral in eq. (5.19). The contribution to the anomalous dimension is then

$$
\gamma_{\mathrm{C} / \mathrm{A}}^{\mathrm{A}, \alpha_{s}^{2}}=\left(\frac{\alpha_{s}}{4 \pi}\right)^{2} 34.01 C_{F}^{2}
$$

\subsubsection{Two-loop anomalous dimension and comparison with EVENT2}

Combining eqs. (5.13), (5.17) and (5.22), the total two-loop non-cusp anomalous dimension for the soft function is

$$
\gamma_{S}^{\alpha_{s}^{2}}=\left(\frac{\alpha_{s}}{4 \pi}\right)^{2} C_{F}\left[34.01 C_{F}+\left(\frac{1616}{27}-56 \zeta_{3}-9.31\right) C_{A}-\left(\frac{448}{27}+14.04\right) n_{f} T_{R}-\frac{2 \pi^{2}}{3} \beta_{0}\right] .
$$

The two-loop non-cusp anomalous dimension for the collinear-soft function is found by consistency using eq. (5.4). Note that this anomalous dimension has no $\log z_{\text {cut }}$ terms. Therefore, the anomalous dimensions of no functions in the factorization theorem have $\log z_{\text {cut }}$ dependence. This is a consequence of the fact that each function of our factorization theorem in eq. (3.10) depends on a single infrared scale, allowing NNLL resummation of all logarithms of $z_{\text {cut }}$ and $e_{2}^{(2)}$ alike. As we discuss in sections 3.3 and 5.2, this result relies on the choice of Cambridge/Aachen reclustering in the soft drop algorithm.

We can verify this result by comparing the resummed distribution, truncated at $\mathcal{O}\left(\alpha_{s}^{2}\right)$, with the singular region of the full QCD result, computed to the same fixed order. For the full QCD result, we have implemented soft drop into EVENT2 [35], a Monte Carlo code that generates fixed-order results up to $\mathcal{O}\left(\alpha_{s}^{2}\right)$ in $e^{+} e^{-}$collisions. Our specific implementation is as follows. We generate $e^{+} e^{-}$collisions at $1 \mathrm{TeV}$ center of mass energy and identify event hemispheres with the exclusive $k_{T}$ algorithm [45]. We then recluster each hemisphere using the Cambridge/Aachen algorithm and apply soft drop with $\beta=0$. On each of the soft-drop groomed hemispheres, we then measure the energy correlation function $e_{2}^{(2)}$ and record the larger of the two values, which we denote by $e_{2, H}^{(2)}$ and refer to as the heavy groomed mass. This is simply related to the cross section of our factorization theorem:

$$
\frac{d \sigma}{d e_{2, H}^{(2)}}=\int d e_{2, L}^{(2)} d e_{2, R}^{(2)} \frac{d^{2} \sigma}{d e_{2, L}^{(2)} d e_{2, R}^{(2)}}\left[\Theta\left(e_{2, L}^{(2)}-e_{2, R}^{(2)}\right) \delta\left(e_{2, H}^{(2)}-e_{2, L}^{(2)}\right)+(L \leftrightarrow R)\right] .
$$

In figure 4, we compare EVENT2 results to the prediction of the factorized expression at NNLL expanded to $\mathcal{O}\left(\alpha_{s}^{2}\right)$. For soft drop with $\beta=0$, soft logarithms are removed, which means that at $\mathcal{O}\left(\alpha_{s}^{2}\right)$, the cross section has the schematic form

$$
e_{2, H}^{(2)} \frac{d \sigma^{\alpha_{s}^{2}}}{d e_{2, H}^{(2)}} \sim \alpha_{s}^{2} C_{0} \log e_{2, H}^{(2)}+\alpha_{s}^{2} C_{1}
$$




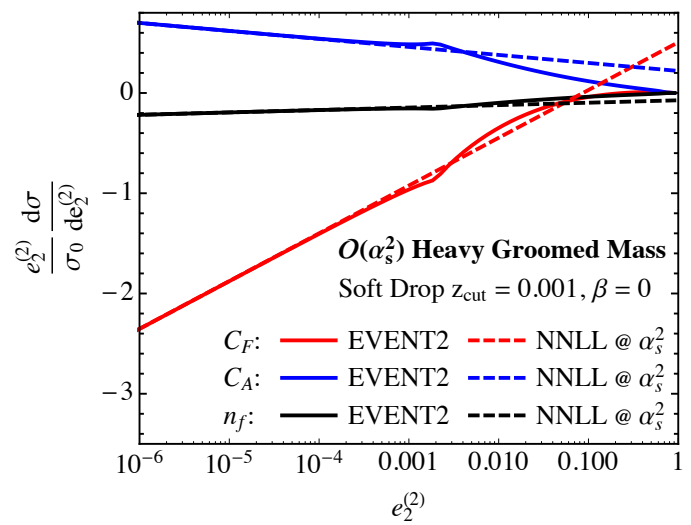

(a)

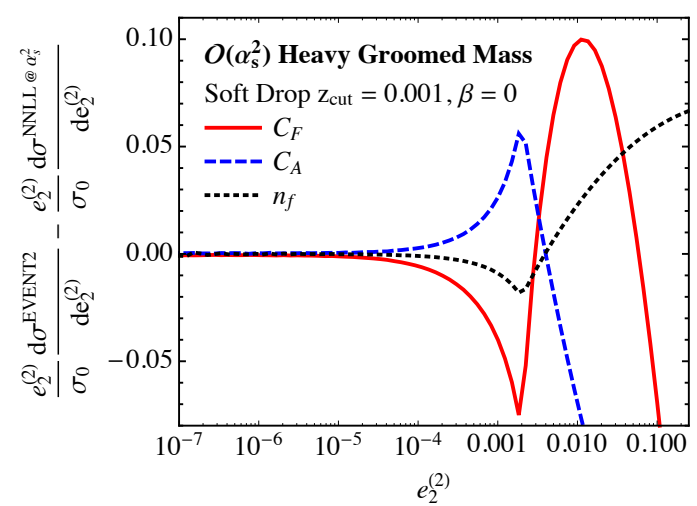

(b)

Figure 4. Verification of our factorization theorem at $\mathcal{O}\left(\alpha_{s}^{2}\right)$ for soft-drop grooming with $z_{\text {cut }}=$ 0.001 and $\beta=0$. Solid curves are numerical results from EVENT2, and dashed curves are $\mathcal{O}\left(\alpha_{s}^{2}\right)$ terms in our NNLL distribution, plotted in the three color channels $C_{F}^{2}, C_{F} C_{A}$, and $C_{F} n_{f} T_{R}$. (a) shows a direct comparison and (b) the difference.

where $C_{0}$ and $C_{1}$ are constants. We plot the cross section separated into the three color channels $\left(C_{F}^{2}, C_{F} C_{A}\right.$, and $\left.C_{F} n_{f} T_{R}\right)$. We set $z_{\text {cut }}=0.001$ to suppress power corrections of $z_{\text {cut }}$. Excellent agreement between our factorization theorem and EVENT2 is observed at small $e_{2}^{(2)}$, demonstrating that we have captured all singular terms of the full QCD result in our factorization theorem to $\mathcal{O}\left(\alpha_{s}^{2}\right)$.

\subsection{Reclustering with anti- $k_{T}$}

It is illuminating to study the clustering effects in the soft function in more detail. In this section, we re-calculate the clustering effects with the anti- $k_{T}$ algorithm, instead of the standard Cambridge/Aachen algorithm. We find that the clustering effects with the anti- $k_{T}$ algorithm are intimately related to the corresponding effects calculated in jet veto calculations. This can be understood relatively simply by re-expressing the clustering conditions in a form analogous to the clustering metric of the longitudinally-invariant $k_{T}$ algorithm.

To calculate the two-loop soft function for soft drop defined with anti- $k_{T}$ reclustering, we only need to calculate the clustering effects unique to this algorithm. We will denote the phase space constraints for the anti- $k_{T}$ reclustering as $\Theta_{\mathrm{SD}}^{\mathrm{a} k_{T}}$, but we will not explicitly present them here. The two-loop soft function is

$$
\left.S^{\mathrm{a} k_{T}}\left(z_{\mathrm{cut}}\right)\right|_{\alpha_{s}^{2}}=\int\left[d^{d} k_{1}\right]_{+}\left[d^{d} k_{2}\right]_{+}\left|\mathcal{M}\left(k_{1}, k_{2}\right)\right|^{2} \Theta_{\text {veto }}+\int\left[d^{d} k_{1}\right]_{+}\left[d^{d} k_{2}\right]_{+}\left|\mathcal{M}\left(k_{1}, k_{2}\right)\right|^{2}\left[\Theta_{\mathrm{SD}}^{\mathrm{a} k_{T}}-\Theta_{\text {veto }}\right] .
$$

The relevant phase space constraints can be written as

$$
\begin{aligned}
\Theta_{\mathrm{SD}}^{\mathrm{a} k_{T}}-\Theta_{\text {veto }}= & \Theta\left(\eta_{1} \eta_{2}\right)\left[1-\Theta\left(\max \left[k_{1}^{0}, k_{2}^{0}\right] \theta_{1 J}-\frac{Q}{2} \theta_{12}\right) \Theta\left(\max \left[k_{1}^{0}, k_{2}^{0}\right] \theta_{2 J}-\frac{Q}{2} \theta_{12}\right)\right] \\
& \times \Theta\left(z_{\text {cut }} \frac{Q}{2}-k_{1}^{0}\right) \Theta\left(z_{\text {cut }} \frac{Q}{2}-k_{2}^{0}\right) \Theta\left(k_{1}^{0}+k_{2}^{0}-z_{\text {cut }} \frac{Q}{2}\right) .
\end{aligned}
$$


With this, we can calculate the divergent part of the two-loop soft function from clustering effects and extract the anomalous dimension. As with Cambridge/Aachen, we can write the two-loop non-cusp anomalous dimension as

$$
\gamma_{S}^{\mathrm{a} k_{T}}=\gamma_{\mathrm{veto}}+\gamma_{\mathrm{a} k_{T}}
$$

where $\gamma_{\mathrm{a} k_{T}}$ is the part of the anomalous dimension purely from clustering effects. We find

$$
\begin{aligned}
\gamma_{\mathrm{a} k_{T}}=-8\left(\frac{\alpha_{s}}{4 \pi}\right)^{2} & C_{F}\left\{\left[\left(\frac{131}{9}-\frac{4}{3} \pi^{2}-\frac{44}{3} \log 2\right) C_{A}+\left(-\frac{46}{9}+\frac{16}{3} \log 2\right) n_{f} T_{R}\right] \log z_{\text {cut }}\right. \\
+ & \left(-\frac{269}{6}+\frac{7}{2} \zeta_{3}+\frac{274}{9} \log 2+\frac{11 \pi^{2}}{9}+\frac{44}{3} \log ^{2} 2\right) C_{A} \\
+ & \left.\left(\frac{53}{3}-\frac{4 \pi^{2}}{9}-\frac{116}{9} \log 2-\frac{16}{3} \log ^{2} 2\right) n_{f} T_{R}\right\} .
\end{aligned}
$$

This anomalous dimension is fascinating. First, note that there is no $C_{F}^{2}$ term, implying that non-Abelian exponentiation holds for anti- $k_{T}$ reclustering, in contrast to what we found for the Cambridge/Aachen algorithm. That is, all logarithms at $\mathcal{O}\left(\alpha_{s}^{2}\right)$ with color factor $C_{F}^{2}$ are accounted for by exponentiating the one-loop result. This is to be expected: since the anti- $k_{T}$ algorithm clusters soft gluons (with energy fractions of order $z_{\text {cut }}$ ) oneby-one with the hard jet core unless two soft gluons have angular separation $\Delta R \lesssim z_{\text {cut }}$, clustering effects are merely a power correction for Abelian gluons.

Also, unlike the case for Cambridge/Aachen reclustering, there is explicit $\log z_{\text {cut }}$ dependence in the anomalous dimension of eq. (5.29). This shows that we do not resum logarithms of $z_{\text {cut }}$ to full NNLL accuracy when anti- $k_{T}$ clustering is used in soft drop. The coefficient of the $\log z_{\text {cut }}$ term is identical to the coefficient of the logarithm of the jet radius $R$ found from clustering effects in jet veto calculations [30-34]. This connection between soft drop and jet veto calculations can be made clearer by a simple rewriting of the clustering metric.

The $k_{T}$ class of clustering metrics for $e^{+} e^{-}$collisions can be written as

$$
d_{i j}=\min \left[E_{i}^{2 p}, E_{j}^{2 p}\right] \theta_{i j}^{2},
$$

for particles $i$ and $j$, with $p$ an integer that defines the jet algorithm. In the soft function, soft particles are either clustered with each other or with the jet axis. For $\beta=0$, these soft particles have characteristic energy fraction $z_{\text {cut }} \ll 1$. In terms of energy fractions, the clustering metric of two soft particles is

$$
d_{i j}=\min \left[z_{i}^{2 p}, z_{j}^{2 p}\right] Q^{2 p} \theta_{i j}^{2} \sim z_{\text {cut }}^{2 p} Q^{2 p} \theta_{i j}^{2},
$$

and for a soft particle $i$ with the jet axis it is

$$
d_{i}=\min \left[1, z_{\text {cut }}^{2 p}\right] Q^{2 p} \theta_{i}^{2}
$$

where $\theta_{i}$ is the angle between particle $i$ and the jet axis. 
Consider $p<0$. In this case, the two soft gluons are (parametrically) clustered together when

$$
z_{\text {cut }}^{p} \theta_{i j}<\min \left[\theta_{i}, \theta_{j}\right]
$$

or equivalently, when

$$
\theta_{i j}<z_{\text {cut }}^{|p|} \min \left[\theta_{i}, \theta_{j}\right]
$$

The effective clustering metric in this case is then

$$
d_{i j}^{\mathrm{eff}}=\min \left[z_{i}^{2 p}, z_{j}^{2 p}\right] \frac{\theta_{i j}^{2}}{z_{\text {cut }}^{2|p|} \min \left[\theta_{i}^{2}, \theta_{j}^{2}\right]}, \quad d_{i}^{\mathrm{eff}}=z_{i}^{2 p} .
$$

With $p=-1$, this is the clustering metric for the inclusive anti- $k_{T}$ algorithm with effective jet radius $R=z_{\text {cut }} \ll 1$. There will now be logarithms of the jet radius that arise. The $\log z_{\text {cut }}$ term in the anomalous dimension has the identical coefficient as the $\log R$ term in jet veto calculations because $z_{\text {cut }}$ and $R$ act as the angular scale for collinear splittings in the respective soft functions.

In summary, while we could use anti- $k_{T}$ to recluster the jet for soft drop grooming, we could not resum all large logarithms to the same precision without a different factorization theorem. Therefore, reclustering in soft drop with the Cambridge/Aachen algorithm is preferred from a theory perspective.

\subsection{NNLL for $\alpha=2, \beta \geq 0$}

For soft drop with angular exponent $\beta>0$, we cannot recycle results from the literature to reach NNLL precision. Instead, a completely new two-loop calculation of either the soft or collinear-soft function is needed. But without such a calculation, we can perform NNLL resummation for particular values of $\beta>0$, using numerical simulations to estimate the ingredients we lack. We will demonstrate this explicitly in the case of $\beta=1$, and the result will allow us to study features of NNLL distributions for energy correlation functions with less aggressive grooming.

The same method we used to validate anomalous dimensions for $\beta=0$ can be used to extract the anomalous dimension for $\beta>0$. This method relies on the fact that all ingredients necessary for NNLL resummation with $\alpha=2, \beta>0$ are known except the two-loop non-cusp anomalous dimensions of the soft and collinear-soft functions. As mentioned above, renormalization group invariance determines one of these, say $\gamma_{S_{C}}^{(1)}$, in terms of the other anomalous dimensions. So only one unkown, $\gamma_{S}^{(1)}$, remains and we can extract it at fixed order.

To do this for a given $\beta>0$, we can use EVENT2 to obtain numerical results at $\mathcal{O}\left(\alpha_{s}^{2}\right)$ for the groomed $e_{2, H}^{(2)}$ distribution with several moderately small values of $z_{\text {cut }}$. From each of these distributions, we can subtract the known terms, which we get by expanding the NNLL distribution to fixed order. This leaves a term proportional to the unknown $\gamma_{S}^{(1)}$, as well as power corrections suppressed by $e_{2, H}^{(2)}$ or $z_{\text {cut }}$. By computing the distribution down to very small $e_{2, H}^{(2)}$, we can ignore the $e_{2, H}^{(2)}$ power corrections. Reducing power corrections from $z_{\text {cut }}$ is limited by the numerical precision of EVENT2 because our factorization theorem only 


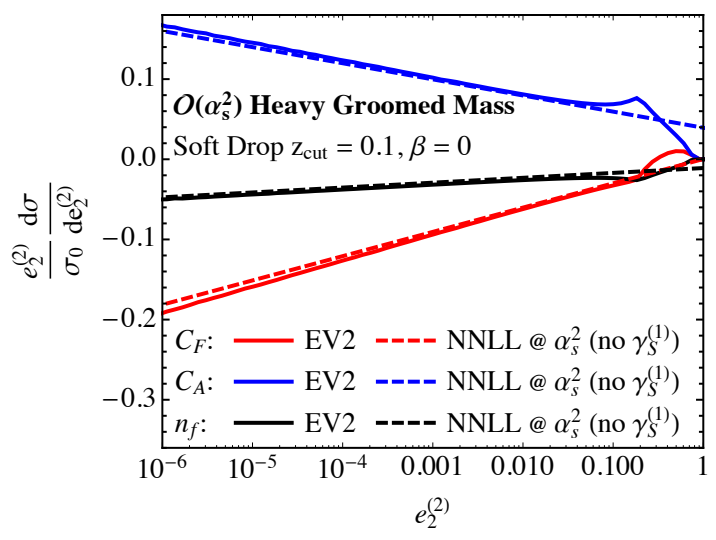

(a)

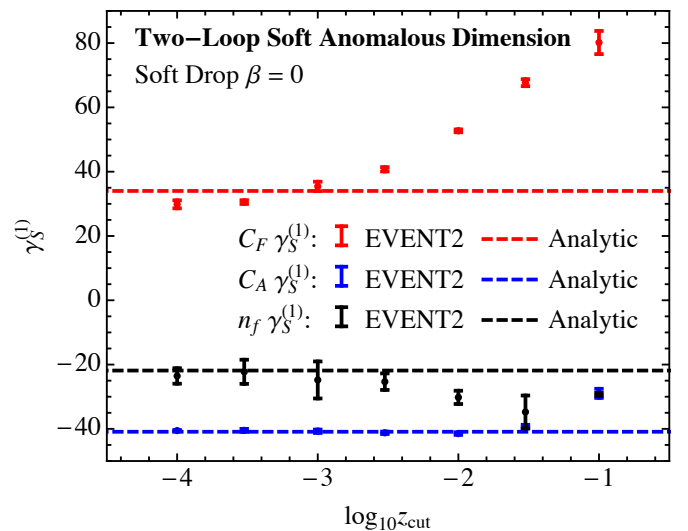

(b)

Figure 5. Demonstration of non-cusp anomalous dimension extraction in EVENT2. (a) Solid curves are numerical results from EVENT2 at $\mathcal{O}\left(\alpha_{s}^{2}\right)$ with $\beta=0$ and $z_{\text {cut }}=0.1$. Dashed curves are $\mathcal{O}\left(\alpha_{s}^{2}\right)$ terms in NNLL distribution, without the term proportional to $\gamma_{S}^{(1)}$. Discrepancy results from $z_{\text {cut }}$ power corrections in solid curves and missing $\gamma_{S}^{(1)}$ in dashed curves. Subtracting $z_{\text {cut }} \log ^{n}\left(z_{\text {cut }}\right) \log \left(e_{2, H}^{(2)}\right)$ power corrections from dashed curves, we extract the remaining offsets. (b) As $z_{\text {cut }} \rightarrow 0$, remaining offsets allow extraction of $\gamma_{S}^{(1)}$ in rough agreement with eq. (5.23).

applies for $e_{2, H}^{(2)} \ll z_{\text {cut }}$. Instead, we can fit the $z_{\text {cut }}$ power corrections to linear combinations of $z_{\text {cut }} \log ^{n}\left(z_{\text {cut }}\right) \log ^{m}\left(e_{2, H}^{(2)}\right)$. At $\mathcal{O}\left(\alpha_{s}^{2}\right)$ it is appropriate to use $0 \leq n+m \leq 3$, though in practice we found terms with $m \geq 2$ to be difficult to fit. With the non-negligible power corrections thus removed, we can then extract the remaining anomalous dimension.

While the procedure outlined above is straightforward, an explicit calculation of $\gamma_{S}^{(1)}$ or $\gamma_{S_{C}}^{(1)}$ for $\beta>0$ is of course desirable. On practical time scales, numerical extractions are limited to rough approximations, due to inadequate numerical precision in the deep infrared. Nevertheless, an estimate is sufficient for our purposes here, which are to demonstrate the advantages of resumming jet substructure observables to NNLL, and to examine various levels of grooming. Thus, we will test the above procedure on $\beta=0$, and learn about the associated uncertainties by comparing with our direct calculation, eq. (5.23). Then we will move to $\beta=1$, and extract $\gamma_{S}^{(1)}$ in that case.

In figure 5 a we show numerical results at $\mathcal{O}\left(\alpha_{s}^{2}\right)$ from EVENT2 with $\beta=0$ and $z_{\text {cut }}=0.1$. Also shown is the NNLL distribution, expanded to fixed order, but without the $\gamma_{S}^{(1)}$ term. The discrepancy between the curves is thus due to the missing $\gamma_{S}^{(1)}$ term and $z_{\text {cut }}$ power corrections. Using several distributions like this one, with values of $z_{\text {cut }}$ between $10^{-4}$ and $10^{-1}$, we fit the $z_{\text {cut }}$ power corrections. Figure $5 \mathrm{~b}$ shows the remaining offsets between our analytical curves and the results of EVENT2, after $z_{\text {cut }} \log ^{n}\left(z_{\text {cut }}\right) \log \left(e_{2, H}^{(2)}\right)$ power corrections have been subtracted. On each point in this plot, the error bar represents the standard deviation in EVENT2 output, across $e_{2, H}^{(2)}$ bins. The offset that remains as $z_{\text {cut }} \rightarrow 0$ is the $\gamma_{S}^{(1)}$ we would extract using this method. One can see from figure $5 \mathrm{~b}$ that agreement with our analytical calculation, eq. (5.23), is quite good, with some discrepancy in the $C_{F}$ channel. 


\begin{tabular}{|c|ccc|}
\hline Soft Drop $\gamma_{S}^{(1)}$ & $C_{F}$ & $C_{A}$ & $n_{f}$ \\
\hline$\beta=0$ extraction & $28 \pm 1.5$ & $-40 \pm 1$ & $-23 \pm 3$ \\
\hline$\beta=0$ calculation & 34.01 & -40.90 & -21.86 \\
\hline$\beta=1$ extraction & $6 \pm 12$ & $-9.5 \pm 2$ & $-8 \pm 7$ \\
\hline
\end{tabular}

Table 2. Extraction of two-loop non-cusp anomalous dimension $\gamma_{S}^{(1)}$ of wide-angle soft function in the three different color channels. For $\beta=0$ comparison with our direct calculation is possible. See text for discussion of uncertainties.

Table 2 lists the numerical results of $\gamma_{S}^{(1)}$ using this method. ${ }^{5}$ The uncertainties quoted for the $\beta=0$ extraction in the table come from the standard deviation in EVENT2 output across $e_{2, H}^{(2)}$ bins, which introduces an error in the identification of constant offsets. These should be compared with our direct calculation in the second line of the table. The discrepancy in the $C_{F}$ channel gives us a sense of additional numerical uncertainties, which are significant. Similar disagreements have been encountered before, e.g. in ref. [82], in the context of $C_{F}$ channel extractions from EVENT2, and to resolve it might require significantly longer run times.

As stated above, a rough estimate of $\gamma_{S}^{(1)}$ for $\beta>0$ is sufficient for our purposes, so we have applied the method described above to the case of $\beta=1$. See the third line of table 2 for the results of the extraction. Uncertainties quoted in this line of the table have two sources: (i) variance in EVENT2 output, and (ii) additional numerical precision issues, which we took to be the difference (both absolute and relative) between extraction and direct calculation in the $\beta=0$ test. In each color channel, we took the maximum of these uncertainties and inflated it by a factor of 2 .

The estimate in table 2 allows us to study NNLL distributions of $e_{2, H}^{(2)}$ groomed with $\beta=1$. In the resulting distributions, the uncertainties associated with the imperfect extraction are relatively small; e.g. see figure $6 \mathrm{~b}$ below. Still, a direct calculation of either $\gamma_{S}^{(1)}$ or $\gamma_{S_{C}}^{(1)}$ for $\beta>0$ would of course be preferred, but we leave this to future work.

\section{Matching NNLL to fixed order in $e^{+} e^{-} \rightarrow$ dijets}

Using the results calculated in the previous sections, here we match our resummed differential cross section for soft-drop groomed energy correlation functions to fixed-order for hemisphere jets produced in $e^{+} e^{-}$collisions. We first match resummed results at NLL and NNLL to $\mathcal{O}\left(\alpha_{s}\right)$ and $\mathcal{O}\left(\alpha_{s}^{2}\right)$, respectively, using EVENT2 and demonstrate that theoretical uncertainties are greatly reduced at NNLL. We then compare several Monte Carlo parton shower simulations to our matched NNLL results. We compare both parton and hadron level Monte Carlo to our perturbative analytic results, and include a simple model

\footnotetext{
${ }^{5}$ In carrying out the procedure just described, we tuned EVENT2 parameters to favor the infrared. In particular, we use of order 1 trillion events, with CUTOFF $=10^{-15}$ and phase-space sampling exponents NPOW1 $=$ NPOW2 $=5$. This procedure corresponded to centuries of CPU time.
} 
of hadronization in our calculation. We leave a detailed understanding and justification of incorporating hadronization into the resummed and matched cross section to future work.

\subsection{Matching resummation to fixed-order}

With the explicitly calculated and extracted two-loop non-cusp anomalous dimensions of the soft function in the soft drop factorization theorem eq. (3.10), we are able to resum the differential cross section through NNLL accuracy in the region where $e_{2}^{(2)} \ll z_{\text {cut }} \ll 1$. Anomalous dimensions of all functions are collected in the appendices and we present the explicit form of the resummed cross section in appendix F. This resummed cross section is only valid in the region where $e_{2}^{(2)} \ll z_{\text {cut }} \ll 1$, and will not provide an accurate description of the cross section outside this region. To accurately describe the cross section throughout the full phase space requires matching the resummed result to fixed-order.

While there are many ways to do this at various levels of sophistication, we choose to use simple additive matching. That is, we construct matched distributions according to

$$
\frac{d \sigma_{\mathrm{match}}}{d e_{2}^{(2)}}=\frac{d \sigma_{\mathrm{resum}}}{d e_{2}^{(2)}}+\frac{d \sigma_{\mathrm{FO}}}{d e_{2}^{(2)}}-\frac{d \sigma_{\mathrm{resum}, \mathrm{FO}}}{d e_{2}^{(2)}} .
$$

Here, $d \sigma_{\text {resum }}$ is the resummed cross section, calculated to the appropriate logarithmic accuracy. $d \sigma_{\mathrm{FO}}$ is the fixed-order differential cross section calculated to a particular order in $\alpha_{s} . d \sigma_{\text {resum,FO }}$ is the resummed cross section truncated at the same accuracy as the fixed-order cross section. In the infrared phase space region, this term will exactly cancel the singularities in the fixed-order cross section, only leaving the resummed cross section plus power corrections. In the hard phase space region, this term cancels the resummed cross section, up to higher orders in $\alpha_{s}$.

The logarithmic accuracy of the resummed cross section was defined in section 5 , and here we specify the fixed orders that we use in the matching procedure. We additively match the analytic NLL distributions to $\mathcal{O}\left(\alpha_{s}\right)$ fixed order results, which include one real emission from the $q \bar{q}$ dipole. We match NNLL distributions to $\mathcal{O}\left(\alpha_{s}^{2}\right)$ results, which include up to two real emissions. EVENT2 is able to generate $e^{+} e^{-}$collisions through $\mathcal{O}\left(\alpha_{s}^{2}\right)$, except for the two-loop virtual contribution. The two-loop virtual term only contributes at $e_{2}^{(2)}=0$, so our differential distributions are unaffected by this omission.

In figure 6, we plot the resummed and matched differential cross sections for the larger $e_{2}^{(2)}$ of the two hemispheres at NLL and NNLL with various levels of soft drop grooming. Here, we consider dijet production in $e^{+} e^{-}$collisions at $1 \mathrm{TeV}$ center-of-mass energy and identify hemispheres with the exclusive $k_{T}$ algorithm [45]. The parameters of soft drop are $z_{\text {cut }}=0.1$ and we show both $\beta=0$ and $\beta=1$. We also show the ungroomed heavy hemisphere $e_{2}^{(2)}$ distribution. In these plots, we include estimates of theoretical uncertainties represented by the lighter bands about the central curve. While more sophisticated methods for estimating uncertainties exist, we simply vary the natural scales that appear in the functions of the factorization theorem up and down by a factor of two. We then take the envelope of these scale variations as an estimate of theoretical uncertainties. This simple prescription is sufficient for our main purpose in showing uncertainty bands: to demonstrate the reduction in theoretical uncertainty in moving from NLL to NNLL. 


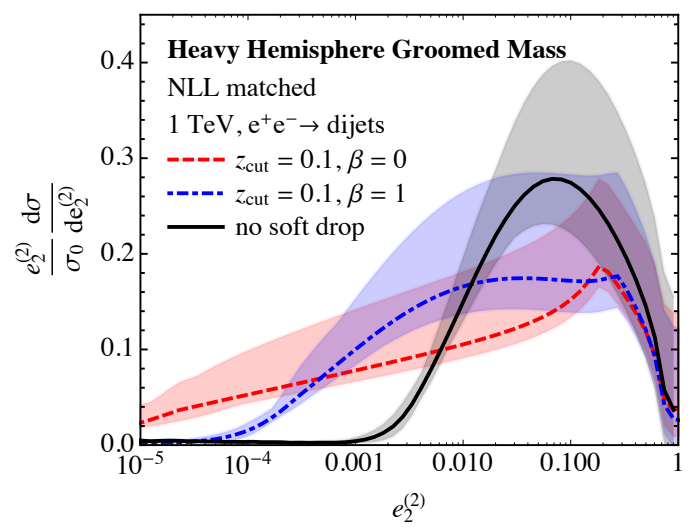

(a)

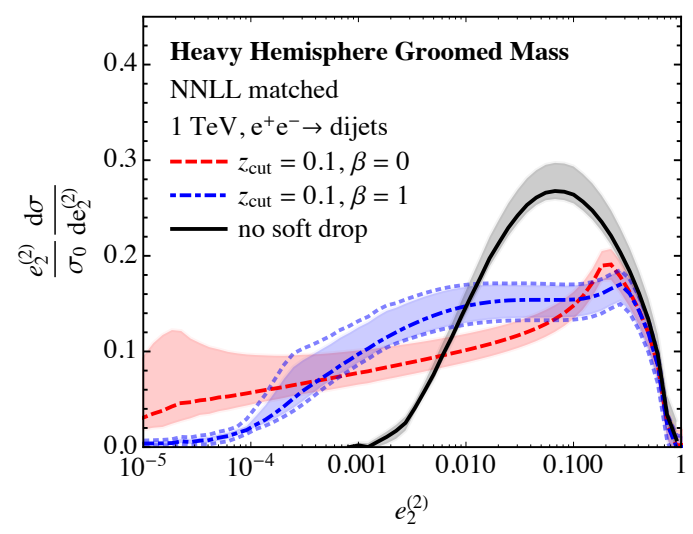

(b)

Figure 6. (a) NLL matched distributions for heavy hemisphere $e_{2}^{(2)}$ in $e^{+} e^{-}$collisions with soft drop grooming $z_{\text {cut }}=0.1$ and $\beta=0, \beta=1$, and without soft drop. Estimates of theoretical uncertainties are represented by the shaded bands. (b) The corresponding matched distributions at NNLL. For soft drop with $\beta=1$, the dotted lines represent the extent of the theoretical uncertainties when the variation of the two-loop non-cusp anomalous dimension is included. Note the significant reduction in uncertainties at NNLL.

Included in these uncertainty estimates is a variation in our treatment of the Landau pole of the strong coupling $\alpha_{s}$. For scales $\mu>1 \mathrm{GeV}, \alpha_{s}$ is evaluated according to its perturbative running. For $\mu<1 \mathrm{GeV}$, we freeze $\alpha_{s}$ to its value at the scale $\mu=1 \mathrm{GeV}$. This is not intended to be a model for hadronization or non-perturbative physics, but is just intended to maintain finite cross section predictions at small $e_{2}^{(2)}$ values. To estimate the sensitivity of our results to the scale at which we freeze the coupling, we vary this $1 \mathrm{GeV}$ scale by a factor of two, and include the effect in the uncertainty bands of figure 6 as well.

Finally, we have shown the uncertainty bands around the $\beta=1$ curves at NNLL with and without the uncertainty in our estimate of the two-loop non-cusp anomalous dimension of the soft function. One can see from the figure that this imperfect extraction has only a relatively small effect on the overall uncertainty at this order.

Importantly, we allow the normalization of the cross section to change under these scale variations. That is, the curves in figure 6 are constructed according to eq. (6.1). The normalization of each distribution displayed is meaningful, since we resum all large logs in both the shape and the normalization. While the central value curves don't change much in going from NLL to NNLL, the uncertainties are dramatically reduced, and this is partly due to the increased accuracy in the normalization.

\subsection{Comparison to Monte Carlo}

In this section, we compare our NNLL resummed and matched soft-drop groomed $e_{2}^{(2)}$ distributions to the output of several standard Monte Carlo simulations. We generate $e^{+} e^{-} \rightarrow$ dijets events at $1 \mathrm{TeV}$ center-of-mass collision energy with HeRWIG ++2.7 .1 [83, 84], Pythia 8.210 [85, 86], and Vincia 1.2 .02 [87-90]. While the Herwig ++ and Pythia events are showered from the leading order process $e^{+} e^{-} \rightarrow q \bar{q}$, we consider VincIA with 


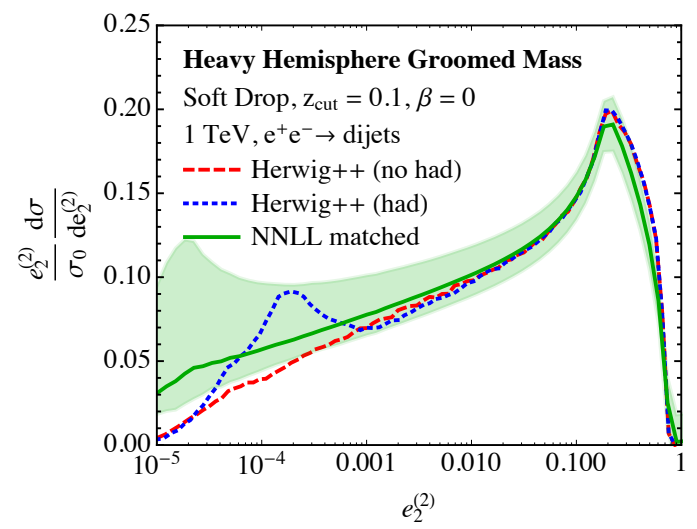

(a)

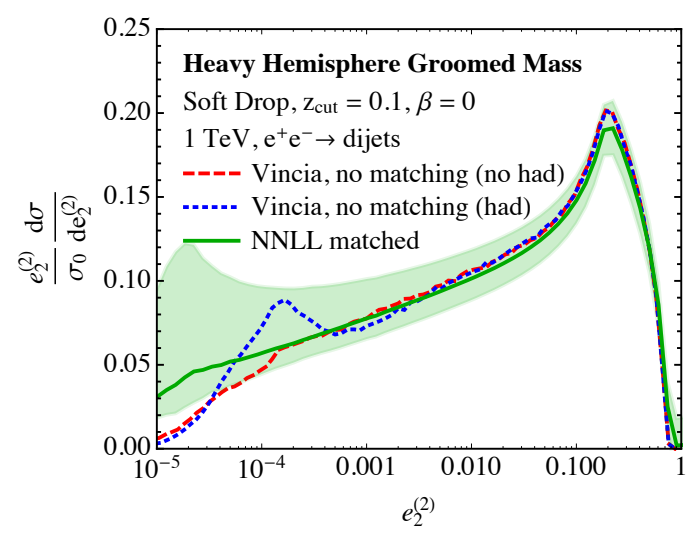

(c)

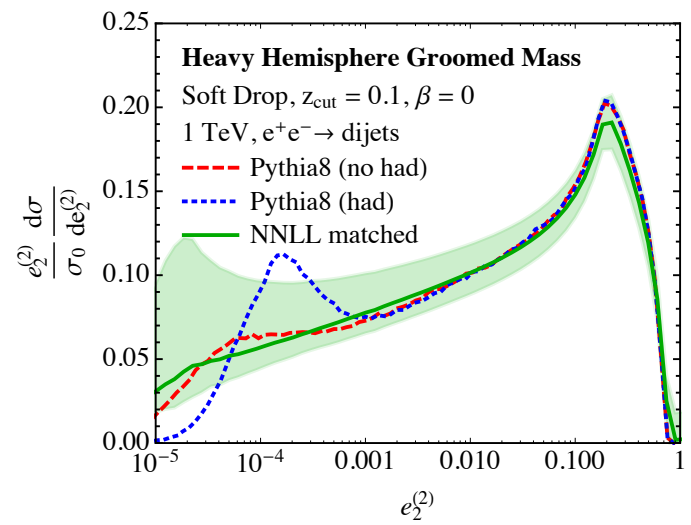

(b)

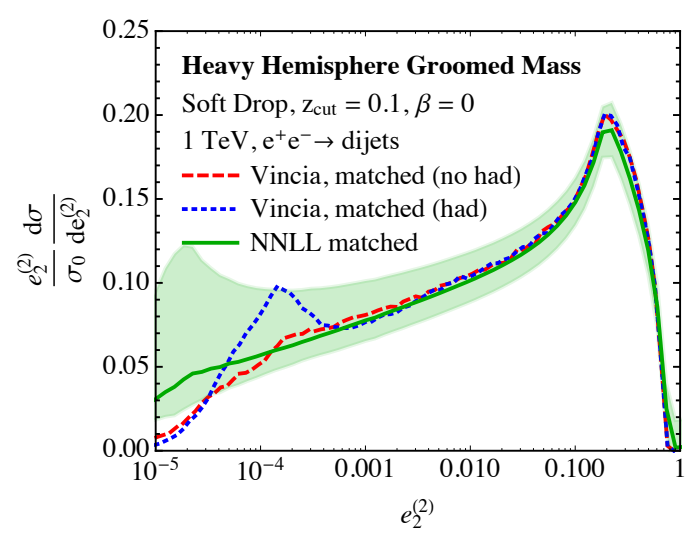

(d)

Figure 7. Comparison between soft-drop groomed $e_{2}^{(2)}$ distributions with $z_{\text {cut }}=0.1$ and $\beta=0$ for NNLL, parton-level, and hadron-level Monte Carlo. All curves integrate to the same value over the range $e_{2}^{(2)} \in[0.01,1]$.

and without fixed-order matching included. The matched VINCIA results are accurate effectively through $\mathcal{O}\left(\alpha_{s}^{2}\right)$. For the most direct comparison of the simulations to our NNLL matched results, we run $\alpha_{s}$ at two loops in all Monte Carlos (in the CMW scheme [91, 92]) and we fix $\alpha_{s}\left(m_{Z}\right)=0.118$, which is the same value used in our analytic calculations. We include Monte Carlo events both at partonic level and after hadronization. These events are then clustered into hemispheres using the exclusive $k_{T}$ algorithm [45] using FASTJET 3.1.3 [93]. The soft drop grooming and subsequent measurement of the two-point energy correlation functions of these $e^{+} e^{-}$events is implemented in FASTJET with custom code.

We compare the Monte Carlo distributions to our NNLL resummed and matched calculations in figures 7 and 8 . In these plots, all distributions integrate to the same value over the range $e_{2}^{(2)} \in[0.01,1]$. In figure 7 , we compare the soft-drop groomed $e_{2}^{(2)}$ distributions with $z_{\text {cut }}=0.1, \beta=0$. Good agreement between the Monte Carlos and our analytic calculation is observed, with (not surprisingly) the matched Monte Carlo agreeing the best. These distributions also show that parton- and hadron-level Monte Carlos are 


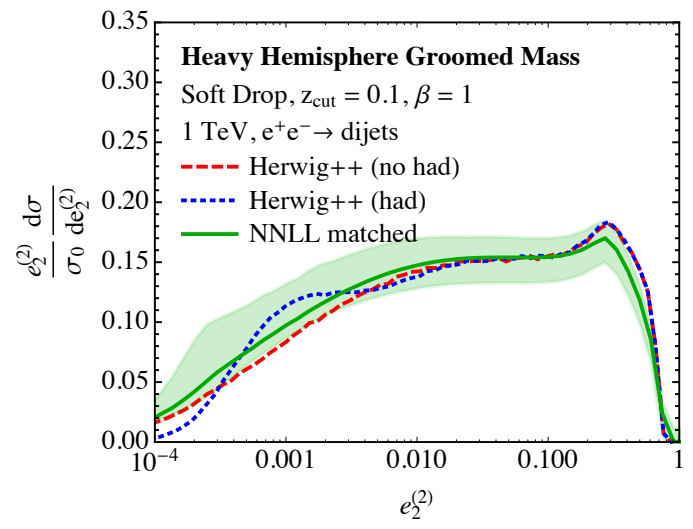

(a)

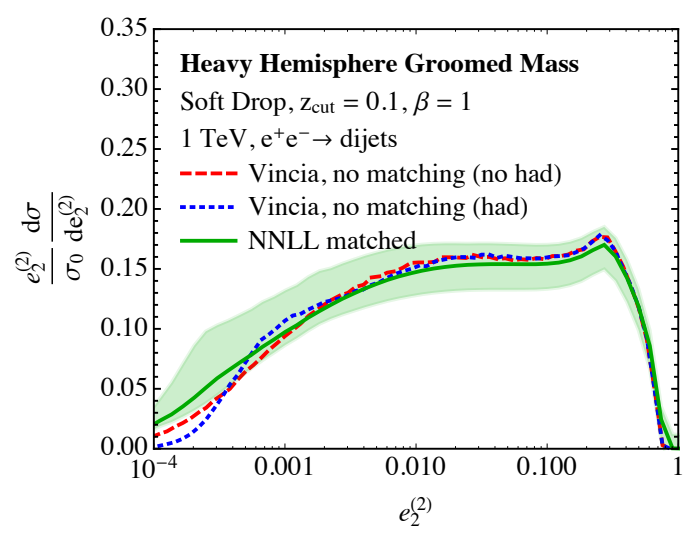

(c)

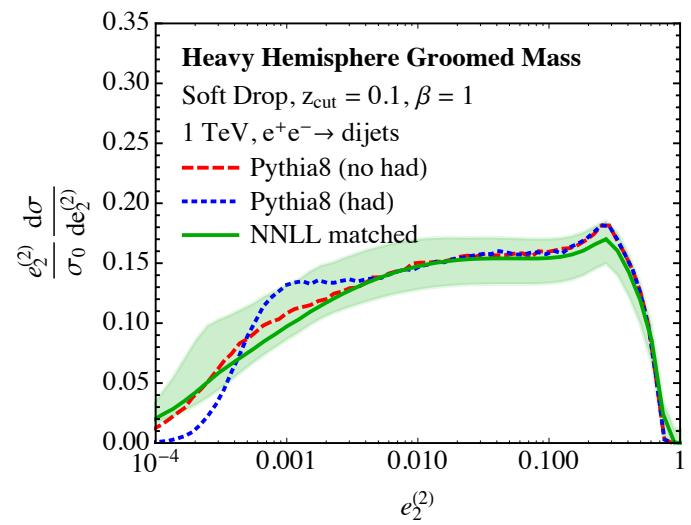

(b)

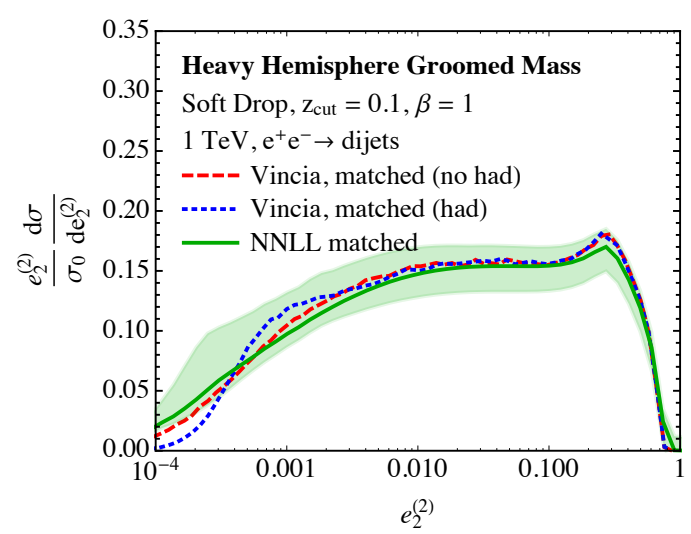

(d)

Figure 8. Comparison between soft-drop groomed $e_{2}^{(2)}$ distributions with $z_{\text {cut }}=0.1$ and $\beta=1$ for NNLL, parton-level, and hadron-level Monte Carlo. All curves integrate to the same value over the range $e_{2}^{(2)} \in[0.01,1]$. The uncertainty band for NNLL includes the variation of the two-loop non-cusp anomalous dimension.

essentially identical for $e_{2}^{(2)} \gtrsim 0.001$. In figure 8 , we compare the soft-drop groomed $e_{2}^{(2)}$ distributions with $z_{\text {cut }}=0.1, \beta=1$. Again, good agreement between the Monte Carlos and our matched NNLL result is observed, with the parton- and hadron-level Monte Carlos nearly identical for $e_{2}^{(2)} \gtrsim 0.005$. The uncertainty bands for the analytic curve includes the uncertainty in the two-loop non- cusp anomalous dimension.

As a more direct comparison of the Monte Carlos, figure 9 displays the relative difference between each of the hadron-level Monte Carlos and our matched NNLL predictions. Again, soft drop is performed with $z_{\text {cut }}=0.1$, and both $\beta=0$ and $\beta=1$ are shown. All the Monte Carlo curves lie within our shaded band of theoretical uncertainty, but discrepancies between the different simulations are visible.

One striking feature in these plots, especially for $\beta=0$, is the presence of additional structure in the hadron-level Monte Carlo distributions at small $e_{2}^{(2)}$. It is clear that this feature is due to non-perturbative physics, and so is therefore not included in our 


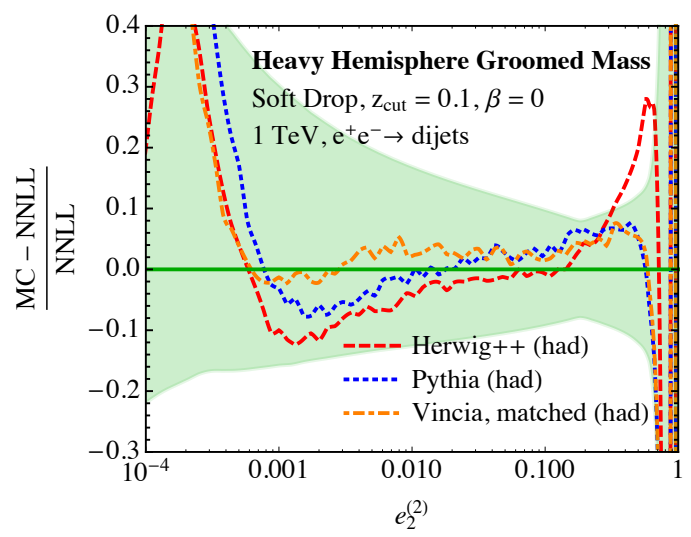

(a)

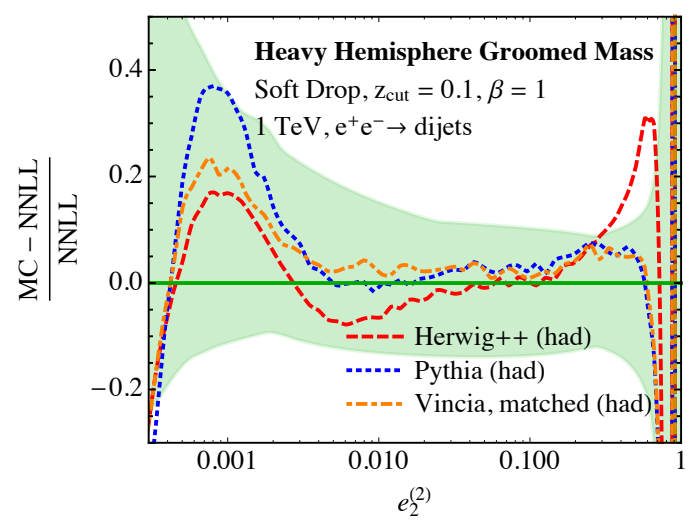

(b)

Figure 9. Direct comparison of hadron-level output from Herwig ++ , PyTHIA, and VinciA already shown in figures 7 and 8 . Soft drop is performed with $z_{\text {cut }}=0.1$ and both $\beta=0$ (left) and $\beta=1$ (right). Curves are displayed as relative differences between Monte Carlo output and our matched NNLL predictions, with theoretical uncertainties shown as a shaded band.

NNLL calculation. Nevertheless, we can include a simple model of hadronization into our calculation to see if this structure is easily explained.

For additive IRC safe observables, like thrust or jet mass, it can be shown from general principles that hadronization corrections can be incorporated in perturbative distributions by convolution with a model shape function [94, 95]. In general, the energy correlation functions are additive observables, so we should be able to use shape functions to model hadronization corrections. However, once soft drop is applied on the jet, emissions in the jet may or may not contribute to the energy correlation functions, so the observable is no longer strictly additive. We leave a more careful study of whether shape functions can be used to model hadronization effects in groomed observables to future work. Here we convolve our matched results with a simple shape function to see if qualitative agreement with the Monte Carlos can be achieved.

Because shape functions describe non-perturbative physics, they only have support for energies comparable to $\Lambda_{\mathrm{QCD}}$. The shape function we choose is the parametrization suggested by ref. [96]:

$$
F_{\text {shape }}(\epsilon)=\frac{4 \epsilon}{\Omega^{2}} e^{-2 \epsilon / \Omega} .
$$

This is normalized

$$
\int_{0}^{\infty} d \epsilon F_{\text {shape }}(\epsilon)=1,
$$

and has first moment equal to $\Omega$. As discussed in section 4.3, of all modes present in our factorization theorem, the collinear-soft mode has the lowest virtuality, so it will have the largest sensitivity to non-perturbative physics. We thus convolve our perturbative distribution with the shape function, assuming non-perturbative effects are primarily associated with the collinear-soft mode. That is, we include hadronization corrections in the soft drop 


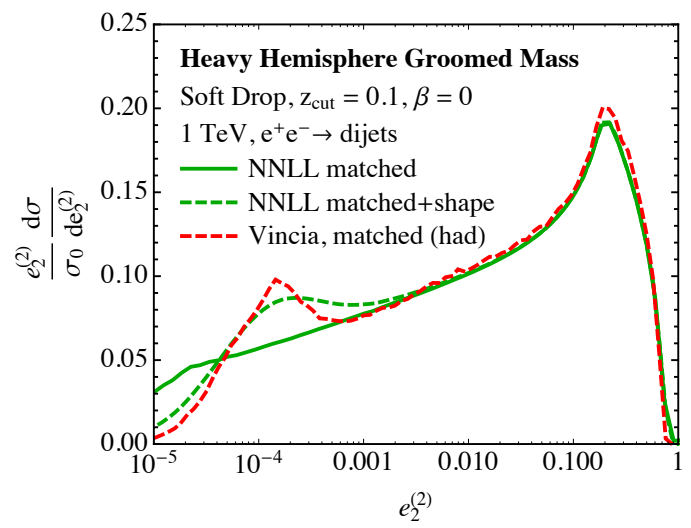

(a)

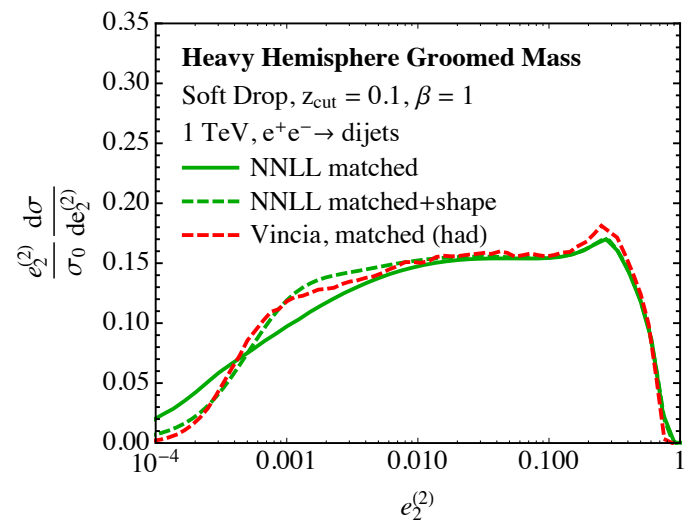

(b)

Figure 10. Perturbative NNLL results for soft-drop groomed $e_{2}^{(2)}$ with $z_{\text {cut }}=0.1$ and $\beta=0$ (left) and $\beta=1$ (right), compared to analytic results that include the shape function of eq. (6.2) for modeling hadronization, and compared to hadron-level Monte Carlo. The parameter $\Omega=1 \mathrm{GeV}$. Note that, qualitatively, the shape function produces a hadronization-bump similar to those seen in the Monte Carlos.

groomed $e_{2}^{(\alpha)}$ distribution according to

$$
\frac{d \sigma_{\text {had }}}{d e_{2}^{(\alpha)}}=\int d \epsilon \frac{d \sigma_{\text {pert }}\left(e_{2}^{(\alpha)}-\left(\frac{\epsilon}{z_{\text {cut }} Q}\right)^{\frac{\alpha-1}{1+\beta}} \frac{\epsilon}{Q}\right)}{d e_{2}^{(\alpha)}} F_{\text {shape }}(\epsilon),
$$

where the argument of the perturbative distribution is shifted by the virtuality of the collinear-soft mode, eq. (4.7).

In figure 10, we compare the matched NNLL distribution of $e_{2}^{(2)}$ with and without convolution with the shape function of eq. (6.2), in which we set $\Omega=1 \mathrm{GeV}$ to be comparable to the scale of hadron masses. We show this comparison for soft drop grooming with $\beta=0$ and $\beta=1$. The peak at small $e_{2}^{(2)}$ for $\beta=0$ agrees qualitatively with the structure of the hadronized Monte Carlo distributions. Similarly, the shape at small $e_{2}^{(2)}$ for $\beta=1$ agrees with the simulations as well. This suggests that there might exist a shape function for describing hadronization effects in groomed jet observables, though we leave a detailed discussion and justification for such a model to future work.

\section{$7 \quad$ Matching NNLL to fixed order in $p p \rightarrow Z+j$}

In this section, we present predictions for soft-drop groomed $e_{2}^{(\alpha)}$ distributions as measured on the jet in $p p \rightarrow Z+j$ events at the LHC. The definitions of soft drop and energy correlation functions appropriate for jets in $p p$ collisions are given in section 2. As with jets from $e^{+} e^{-}$collisions, we match our NNLL resummed distribution to fixed-order results that include relative $\mathcal{O}\left(\alpha_{s}^{2}\right)$ corrections to the Born process.

There are two complications we must deal with. First, at $p p$ collisions, the jets will be both quark and gluon initiated. Second, because we only measure the observable within the 
jet and do not constrain radiation throughout the rest of the event, the simple hard-soft-jet factorization that we employed for $e^{+} e^{-} \rightarrow$ hemisphere jets will not apply here. Nevertheless, while the normalization of the jet-observable distribution will thus be complicated and sensitive to multiple scales, the shape of the distribution will still be controlled exclusively by collinear physics. To address these complications, we first show how soft-drop groomed quark and gluon jets can be unambiguously defined order-by-order in perturbation theory. Then we discuss how the normalization of the distribution can be obtained by matching to full QCD at fixed order. The discussion will focus on the $Z+j$ sample for concreteness, but these ideas apply equally well to any process with hard jets at a hadron collider.

\subsection{Resummed cross section in $p p \rightarrow Z+j$}

We define our observable on soft-drop groomed jets in $p p \rightarrow Z+j$ events in the following way. First, we cluster the final state according to a jet algorithm with some jet radius $R \sim 1$. Of the jets with pseudorapidity $\left|\eta_{J}\right|<\eta_{\max }$, we then identify the jet with the largest transverse momentum $p_{T J}$ and require that $p_{T J}>p_{T}^{\min }$. We groom this jet with soft drop and measure $e_{2}^{(\alpha)}$ according to the definitions given in section 2 for jets in $p p$ collisions. In this procedure, we remain inclusive over all other hadronic activity in the final state: we only care about the hardest jet.

For this process, the relevant factorization formula is

$$
\frac{d \sigma_{\text {resum }}}{d e_{2}^{(\alpha)}}=\sum_{k=q, \bar{q}, g} D_{k}\left(p_{T}^{\min }, \eta_{\max }, z_{\text {cut }}, R\right) S_{C, k}\left(z_{\text {cut }} e_{2}^{(\alpha)}\right) \otimes J_{k}\left(e_{2}^{(\alpha)}\right) .
$$

Unlike our factorization theorem in $e^{+} e^{-}$collisions, eq. (7.1) only resums large logarithms of $e_{2}^{(\alpha)}$ in the limit $e_{2}^{(\alpha)} \ll z_{\text {cut }} \ll 1$. There will be logarithms of $z_{\text {cut }}$ (and other scales in the events) in the $D_{k}$ prefactor that we do not resum. When referring to calculations of this cross section, we will specify the accuracy to which logarithms of $e_{2}^{(\alpha)}$ are resummed (i.e., NLL or NNLL). We now explain the components of this formula in detail.

In eq. (7.1), $S_{C, k}\left(z_{\text {cut }} e_{2}^{(\alpha)}\right)$ and $J_{k}\left(e_{2}^{(\alpha)}\right)$ are the collinear-soft and jet functions for the measurement of soft-drop groomed $e_{2}^{(\alpha)}$ that, by collinear factorization, are identical to the functions defined in $e^{+} e^{-}$collisions. Unlike in $e^{+} e^{-}$collisions, however, these functions also have a label $k$ corresponding to the flavor of the jet, and a sum over the possible QCD parton flavors $k$ is included. The symbol $\otimes$ denotes convolution in $e_{2}^{(\alpha)}$ between the collinear-soft and jet functions.

$D_{k}$ is a matching coefficient that can be extracted from fixed-order calculations, and it sets the normalization and relative contributions from the different jet flavors. In addition to the dependence explicitly shown, $D_{k}$ also depends implicitly on parton distributions, as different initial states produce different flavors of final state jets.

Unlike the case in $e^{+} e^{-}$collisions, where the jet energy was (almost exactly) half the center-of-mass energy, due to the non-trivial parton distributions, the distribution of the jet $p_{T}$ has a finite width and depends on the cut, $p_{T}^{\min }$. For a true precision prediction, we would compute the matching coefficient $D_{k}$ as a function of $p_{T J}$ and include an integral in eq. (7.1) convolving the jet and collinear-soft functions with $D_{k}\left(p_{T J}\right)$. An approach 
to doing this in a semi-automatic manner was discussed recently in refs. [97, 98]. But, for simplicity we instead employ the following approximation: we evaluate the jet and collinear-soft functions at $\bar{p}_{T J}$, the average $p_{T J}$.

The average jet transverse momentum $\bar{p}_{T J}$ can be estimated by using the fact that the cross section for a jet with transverse momentum $p_{T J}$ takes the power-law form:

$$
\frac{1}{\sigma} \frac{d \sigma}{d p_{T J}} \simeq \frac{n-1}{p_{T}^{\min }}\left(\frac{p_{T}^{\min }}{p_{T J}}\right)^{n} \Theta\left(p_{T J}-p_{T}^{\min }\right)
$$

This distribution is normalized and the mean value of $p_{T J}$ is

$$
\bar{p}_{T J}=\frac{n-1}{n-2} p_{T}^{\min } .
$$

The typical exponent is $n \sim 5$, and we take $n=5$ in our numerical computations.

The full cross section for soft-dropped $e_{2}^{(\alpha)}$ (including power corrections) can be expressed as

$$
\frac{d \sigma}{d e_{2}^{(\alpha)}}=\sum_{k=q, \bar{q}, g} D_{k} S_{C, k} \otimes J_{k}+\frac{d \sigma_{\mathrm{pc}}}{d e_{2}^{(\alpha)}} .
$$

Here, the right-most term includes all power corrections suppressed by $e_{2}^{(\alpha)}$ or $z_{\text {cut }}$. The functions $S_{C, k}$ and $J_{k}$ should be evaluated at $\bar{p}_{T J}$ but we have suppressed their arguments for brevity. We will use this form of the cross section to define the matching coefficient $D_{k}$ at fixed-order. For NNLL resummation, the relative $\mathcal{O}\left(\alpha_{s}\right)$ corrections to $D_{k}$ are required.

First, at leading order in $\alpha_{s}$, eq. (7.4) becomes

$$
\frac{d \sigma^{(0)}}{d e_{2}^{(\alpha)}}=\sum_{k=q, \bar{q}, g} D_{k}^{(0)} \delta\left(e_{2}^{(\alpha)}\right),
$$

where the superscript (0) denotes the leading order in $\alpha_{s}$. Here, we have used $J_{k}^{(0)}=$ $S_{C, k}^{(0)}=\delta\left(e_{2}^{(\alpha)}\right)$. Also, since a jet has only one constituent at this order, the distribution has no support away from $e_{2}^{(\alpha)}=0$ and there are no partons to soft drop; therefore, there are no $e_{2}^{(\alpha)}$ or $z_{\text {cut }}$ power corrections at this order. Integrating over all $e_{2}^{(\alpha)}$, we are left with the Born-level cross section for the $k$ flavor channel $\sigma_{k}^{(0)}$, so that

$$
D_{k}^{(0)}=\sigma_{k}^{(0)}
$$

At the next-to-leading order in $\alpha_{s}$, the extraction of $D_{k}$ requires separating the jets by flavor. Since $D_{k}$ is defined in each flavor channel, we need to determine the flavor of the hardest jet in each $p p \rightarrow Z+j$ event included in our sample. Ordinarily, any definition of jet flavor based on the constituents of the jet is infrared-unsafe and ill-defined at leading power, because soft wide-angle emissions into a jet can change its flavor. ${ }^{6}$ Soft drop eliminates this problem at leading power in $e_{2}^{(\alpha)}$ and $z_{\text {cut }}$ by removing soft wide-angle radiation from the jet. This allows for an infrared and collinear safe definition of jet flavor

\footnotetext{
${ }^{6}$ However, one infrared and collinear safe definition of jet flavor was presented in ref. [99].
} 
at leading power in $e_{2}^{(\alpha)}$ and $z_{\text {cut }}$. We define the jet flavor $f_{J}$ as the flavor sum of the constituents of the groomed jet:

$$
f_{J}=\sum_{i \in J_{g}} f_{i}
$$

where $f_{q}=1, f_{\bar{q}}=-1$ and $f_{g}=0$. The subscript on $J_{g}$ means that one only sums over the jet constituents that remain after grooming with soft drop. If $f_{J}= \pm 1$, then the jet is quark-type, while if $f_{J}=0$, it is gluon-type. With this jet flavor identification, we are able to determine the total fixed-order cross section for each jet flavor channel in $p p \rightarrow Z+j$. We will denote the next-to-leading order term in the cross section for a jet of flavor $k$ as $\sigma_{k}^{(1)}$, defined according to the phase space cuts described at the beginning of this section.

Then, at next-to-leading order in the $k$ flavor channel, eq. (7.4) becomes

$$
\frac{d \sigma_{k}^{(1)}}{d e_{2}^{(\alpha)}}=D_{k}^{(0)}\left[S_{C, k}^{(1)}+J_{k}^{(1)}\right]+D_{k}^{(1)} \delta\left(e_{2}^{(\alpha)}\right)+\frac{d \sigma_{k, \mathrm{pc}}^{(1)}}{d e_{2}^{(\alpha)}} .
$$

Here, $S_{C, k}^{(1)}$ and $J_{k}^{(1)}$ are the collinear-soft and jet functions at $\mathcal{O}\left(\alpha_{s}\right)$. Using $D_{k}^{(0)}=\sigma_{k}^{(0)}$, we can integrate over $e_{2}^{(\alpha)}$ to find

$$
D_{k}^{(1)}=\sigma_{k}^{(1)}-\sigma_{k}^{(0)} \int_{0}^{1} d e_{2}^{(\alpha)}\left[S_{C, k}^{(1)}+J_{k}^{(1)}\right]-\sigma_{k, \mathrm{pc}}^{(1)} .
$$

We computed $\sigma_{k}^{(1)}$ using MCFM $[100,101]$ with settings detailed in the next section. We computed the power corrections according to

$$
\sigma_{k, \mathrm{pc}}^{(1)} \equiv \int d e_{2}^{(\alpha)}\left[\frac{d \sigma_{k}^{(1)}}{d e_{2}^{(\alpha)}}-\sigma_{k}^{(0)}\left(J_{k}^{(1)}+S_{C, k}^{(1)}\right)\right] .
$$

For the first term in the integrand, we use a numerical distribution obtained with MCFM. Since we do not have access to this distribution at arbitrarily small values of $e_{2}^{(\alpha)}$, the integral in eq. (7.10) extends from $e_{2}^{(\alpha)}=10^{-5}$ to 1 . This approximation is sufficient for power corrections suppressed by $e_{2}^{(\alpha)}$, and the effect of dropping the $z_{\text {cut }} \delta\left(e_{2}^{(\alpha)}\right)$ term from the integral is negligible in comparison to the scale uncertainties shown in the next section.

This completes our extraction of the matching coefficient $D_{k}$ through relative $\mathcal{O}\left(\alpha_{s}\right)$. With it, the resummed cross section of eq. (7.1) is complete and ready to be matched to relative $\mathcal{O}\left(\alpha_{s}^{2}\right)$ fixed-order results.

\subsection{Matching resummation to fixed-order}

With the resummed differential cross section for soft-drop groomed $e_{2}^{(\alpha)}$ defined in eq. (7.1), we next match to fixed order for $p p \rightarrow Z+j$. Our matching procedure will be identical to the procedure we used for $e^{+} e^{-}$collisions; we add the difference between the exact fixed order and the expansion of the resummed distribution to fixed order:

$$
\frac{d \sigma_{\mathrm{match}}}{d e_{2}^{(\alpha)}}=\frac{d \sigma_{\mathrm{resum}}}{d e_{2}^{(\alpha)}}+\frac{d \sigma_{\mathrm{FO}}}{d e_{2}^{(\alpha)}}-\frac{d \sigma_{\mathrm{resum}, \mathrm{FO}}}{d e_{2}^{(\alpha)}} .
$$


We match the analytic NLL resummed distributions to fixed-order results that include the relative $\mathcal{O}\left(\alpha_{s}\right)$ corrections to the Born process for $p p \rightarrow Z+j$. We match NNLL distributions to fixed-order results including relative $\mathcal{O}\left(\alpha_{s}^{2}\right)$ corrections and up to 3 partons in the jet.

We use MCFM v. $6.8[100,101]$ to generate the fixed-order cross sections for softdrop groomed $e_{2}^{(\alpha)}$ in $p p \rightarrow Z+j$ events. Currently, MCFM can only generate fixedorder corrections at $\mathcal{O}\left(\alpha_{s}\right)$ relative to a Born-level process, and so we will have to use some properties of the observable to be able to calculate to relative $\mathcal{O}\left(\alpha_{s}^{2}\right)$ accuracy. For $e_{2}^{(\alpha)}>0$, as we did in $e^{+} e^{-}$collisions, we can ignore the purely two-loop virtual contribution to $p p \rightarrow Z+j$, as it has no effect on the differential distribution away from $e_{2}^{(\alpha)}=0$. MCFM can generate both inclusive $p p \rightarrow Z+j$ and $p p \rightarrow Z+2 j$ processes through relative $\mathcal{O}\left(\alpha_{s}\right)$ accuracy. Therefore, we can use $p p \rightarrow Z+2 j$ at relative $\mathcal{O}\left(\alpha_{s}\right)$ in MCFM to calculate the relative $\mathcal{O}\left(\alpha_{s}^{2}\right)$ distribution for $p p \rightarrow Z+j$, in the region where $e_{2}^{(\alpha)}>0$.

In practice, this procedure requires some care. To define the cross section for $p p \rightarrow$ $Z+2 j$ in MCFM, we must set a minimum $p_{T}$ for the two jets as identified by MCFM. This is set by the parameter ptjet_min within MCFM. To compute the fixed-order cross section correctly for $e_{2}^{(\alpha)}$ as measured on the soft-drop groomed jet in $p p \rightarrow Z+j$ events, ptjet_min should be set to 0 ; this would of course produce infinity because $p p \rightarrow Z+2 j$ lacks the virtual corrections of $p p \rightarrow Z+j$. To regulate this divergence, we set ptjet_min $=1 \mathrm{GeV}$ and have verified that for jets with $p_{T J}>500 \mathrm{GeV}$, this choice has a negligible effect on the differential cross section of $e_{2}^{(\alpha)}$ until deep in the infrared region, well beyond the point where resummation dominates. Additionally, we have verified that the distribution of $e_{2}^{(\alpha)}$ as measured in $p p \rightarrow Z+j$ at relative $\mathcal{O}\left(\alpha_{s}^{2}\right)$ is identical to that measured in $p p \rightarrow Z+2 j$ at Born level with ptjet_min $=1 \mathrm{GeV}$, up to differences deep in the infrared. Using this procedure, we are therefore able to match to relative $\mathcal{O}\left(\alpha_{s}^{2}\right)$ with MCFM.

We generate $p p \rightarrow Z+j$ events through relative $\mathcal{O}\left(\alpha_{s}^{2}\right)$ accuracy at the $13 \mathrm{TeV}$ LHC using MSTW 2008 NLO parton distribution functions [102]. We require that the $p_{T}$ of the $Z$ boson is greater than $300 \mathrm{GeV}$ and the absolute value of its pseudorapidity is less than 2.5. Jets are clustered with the anti- $k_{T}$ algorithm with radius $R=0.8$. We study the hardest jet in these events that satisfies $p_{T J}>500 \mathrm{GeV}$ and $\left|\eta_{J}\right|<2.5$. On these identified jets, we then soft-drop groom and measure $e_{2}^{(\alpha)}$ using custom code. This is an exceptionally computationally demanding procedure at relative $\mathcal{O}\left(\alpha_{s}^{2}\right)$, due to the complicated phase space of real emissions and the small width of the bins required to calculate the $e_{2}^{(\alpha)}$ distribution. This precision jet substructure study is only possible because of the development of highly efficient methods for generating fixed-order corrections.

In figure 11 we plot matched distributions for soft-drop $e_{2}^{(2)}$ with $z_{\text {cut }}=0.1$ and both $\beta=0$ and $\beta=1$ at NLL and NNLL. Here, we show both the distributions normalized to the total cross section and normalized over the range $e_{2}^{(2)} \in[0.001,0.1]$. The shaded bands represent estimates of theoretical uncertainties due to residual infrared scale sensitivity. ${ }^{7}$

\footnotetext{
${ }^{7}$ The relatively large size of the uncertainty bands for $e_{2}^{(2)} \gtrsim 0.1$ is an artifact of our simplistic additive matching. Additionally, due to the large $K$ factor, the absolute scale of the matched NNLL distribution in figure $11 \mathrm{~b}$ is roughly twice as large as the matched NLL distribution in figure 11a.
} 


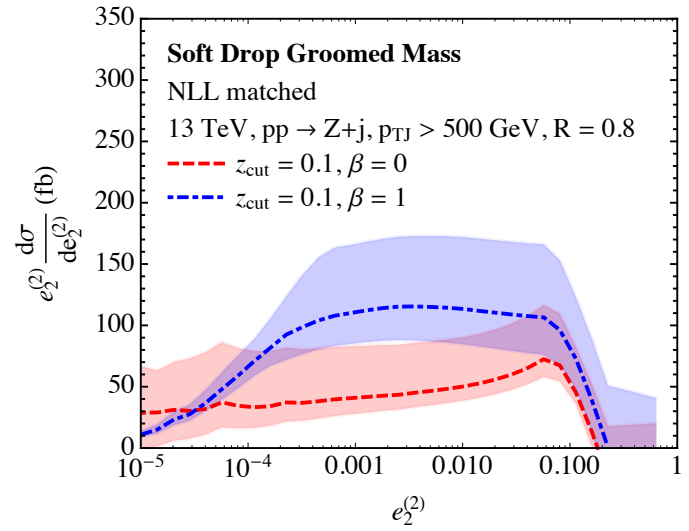

(a)

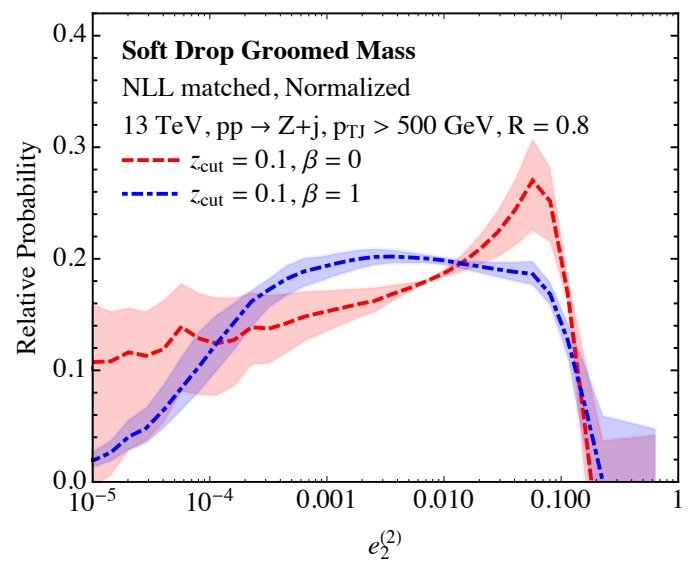

(c)

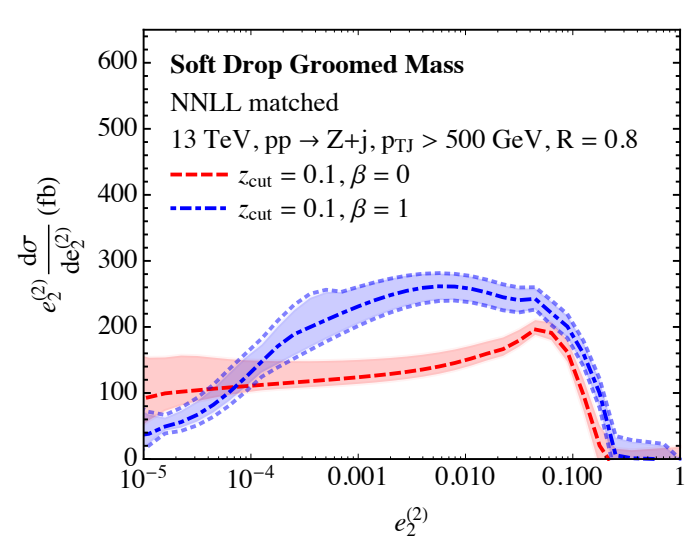

(b)

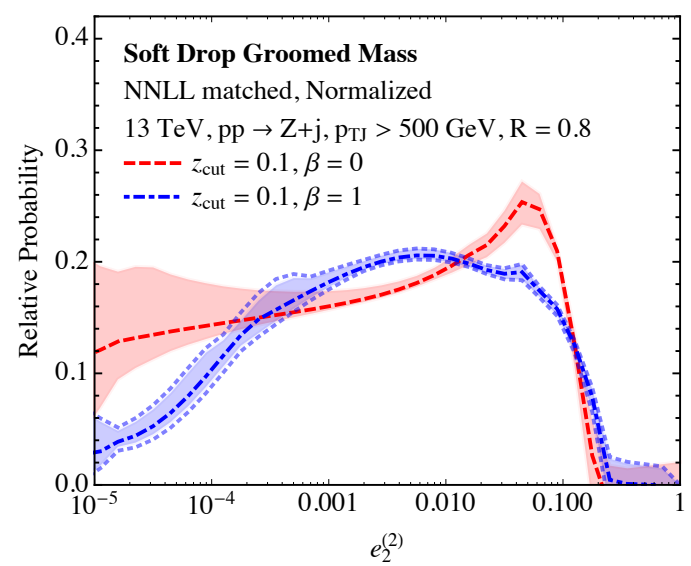

(d)

Figure 11. NLL matched (left) and NNLL matched (right) distributions for hardest jet $e_{2}^{(2)}$ in $p p \rightarrow Z+j$ events with soft drop grooming $z_{\text {cut }}=0.1$ and $\beta=0$ and $\beta=1$. Estimates of theoretical uncertainties are represented by the shaded bands. For soft drop with $\beta=1$, the dotted lines represent the extent of the theoretical uncertainties when the variation of the two-loop noncusp anomalous dimension is included. The distributions in the two upper figures are normalized to the total cross section (in femtobarns), while in the bottom figures, the distributions integrate to the same value over the range $e_{2}^{(2)} \in[0.001,0.1]$. Note the reduction in uncertainties as one moves from NLL to NNLL, and also as one considers the normalized distribution.

We show these bands mainly to allow comparison of the uncertainty remaining at different levels of formal precision. For the collinear-soft and jet functions in the resummed cross section, we vary the low scales by a factor of two. To estimate the scale dependence of the matching coefficient $D_{k}$ in the resummed cross section is more complicated, and we discuss this in detail in appendix G. To estimate scale uncertainties in the fixed-order cross section, we vary the factorization and renormalization scales in MCFM by a factor of 2 about $500 \mathrm{GeV} \simeq p_{T J}$. We then take the envelope of all of these scale variations to produce the shaded bands in figure 11. For $\beta=1$ at NNLL, we have also explicitly shown the additional uncertainty due to the two-loop non-cusp anomalous dimension of the 
collinear-soft function. In going from NLL to NNLL accuracy, the relative size of the scale uncertainty bands decreases by about a factor of 2 or 3 for both choices of normalization of the distributions. However, normalizing the distributions over the range $e_{2}^{(2)} \in[0.001,0.1]$ dramatically reduces residual scale uncertainties; at NNLL, these normalized distributions have residual scale uncertainties at the $10 \%$ level and smaller.

\subsection{Comparison to Monte Carlo}

We now compare our NNLL resummed and matched calculation of soft-drop groomed $e_{2}^{(2)}$ distributions to Monte Carlo simulations. We generate $p p \rightarrow Z+j$ events at the $13 \mathrm{TeV}$ LHC with Herwig ++2.7 .1 and Pythia 8.210. To improve statistics somewhat, we have turned off $Z / \gamma$ interference in the Monte Carlos. The $Z$ boson is forced to decay to electrons, and we require that the invariant mass of the electrons is within $10 \mathrm{GeV}$ of the mass of the $Z$ boson. We then require that the identified $Z$ boson has $p_{T Z}>300 \mathrm{GeV}$ and $\left|\eta_{Z}\right|<2.5$. Jets are clustered with FASTJET 3.1.3 using the anti- $k_{T}$ algorithm with radius $R=0.8$ and we identify the hardest jet in the event with $p_{T J}>500 \mathrm{GeV}$ and $\left|\eta_{J}\right|<2.5$. We then soft-drop groom this jet and measure $e_{2}^{(2)}$. Both soft drop and the energy correlation functions are implemented using FASTJET contrib v. 1.019 [93, 103].

We have generated two samples from both HeRwig ++ and Pythia to study the effect of hadronization and underlying event. One sample is purely parton level: both hadronization and underlying event have been turned off and the other sample is the Monte Carlos run in their default settings, up to the settings of the $Z$ boson mentioned earlier. The distributions of $e_{2}^{(2)}$ measured on soft-drop groomed jets with $z_{\text {cut }}=0.1$ and both $\beta=0,1$ are illustrated in figure 12. Here, we compare our matched and normalized NNLL calculation to both the parton-level and hadron-level plus underlying event Monte Carlos. To normalize the Monte Carlo distributions, all curves integrate to the same value on the range $e_{2}^{(2)} \in[0.001,0.1]$.

As a more direct comparison of the Monte Carlos, figure 13 displays the relative difference between each of the hadron-level Monte Carlos and our matched NNLL predictions, with our estimates of theoretical uncertainty shown as shaded bands. Again, soft drop is performed with $z_{\text {cut }}=0.1$, and both $\beta=0$ and $\beta=1$ are shown. Discrepancies between the Monte Carlo results and our predictions are present but not large.

As observed with jets in $e^{+} e^{-}$collisions, there is good agreement between our precision calculation and the Monte Carlos over a wide dynamic range. Importantly, this measurement of the soft-drop groomed $e_{2}^{(2)}$ is very different from the case in $e^{+} e^{-}$. In $e^{+} e^{-}$ collisions we calculated the heavy groomed and ungroomed jet masses. By measuring the heavier of the two jet masses, both masses have to be small, and the observable is global. For $p p \rightarrow Z+j$ events, we want to make no restrictions on the out-of-jet radiation. Thus although the soft drop jet mass is still free of non-global contributions, the ungroomed mass will not be. That is, we do not have control over all the large logarithms of ungroomed jet mass in $p p \rightarrow Z+j$ events, and thus cannot predict them using our factorized expression, although other approaches are possible. ${ }^{8}$ For this reason, we only show distributions of soft-drop groomed $e_{2}^{(2)}$ measurements in $p p \rightarrow Z+j$ events.

\footnotetext{
${ }^{8}$ Calculations of the ungroomed jet mass in $Z+j$ events have been done, with varying approaches to handing the non-global contribution [41-43].
} 


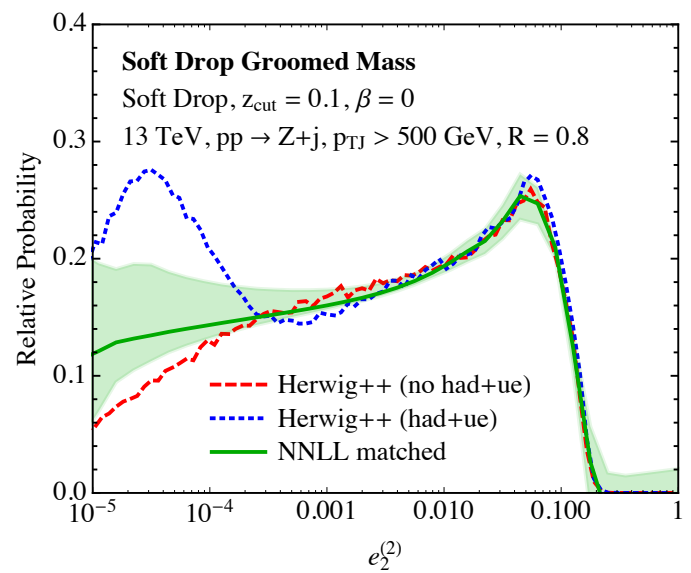

(a)

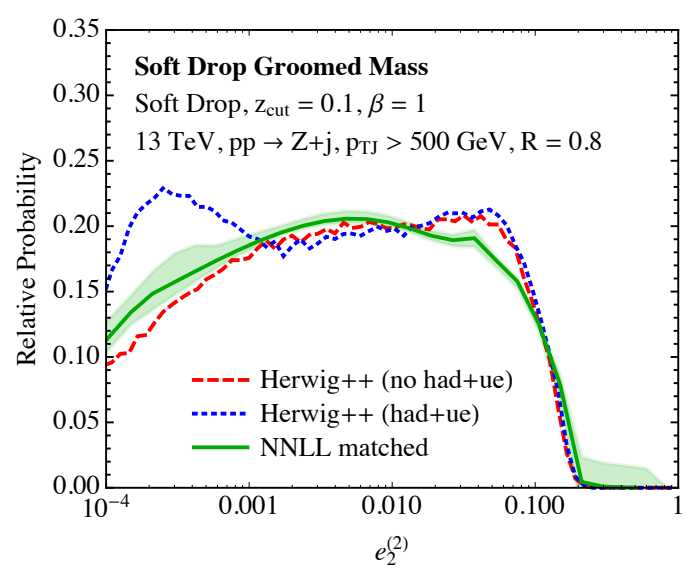

(c)

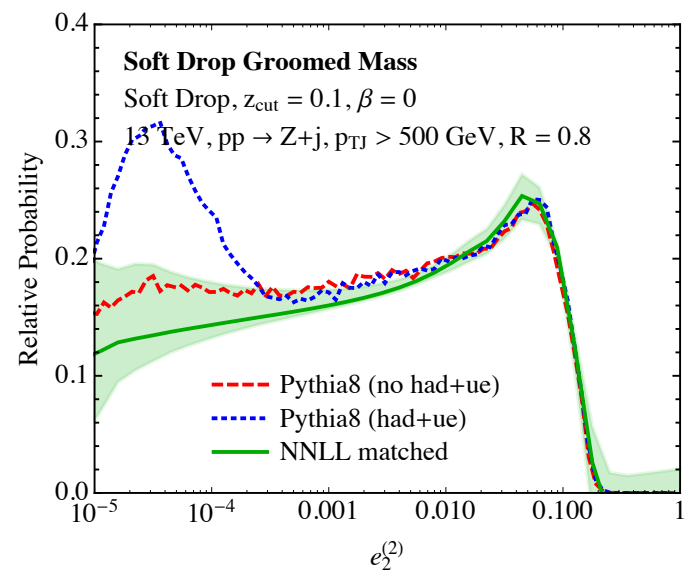

(b)

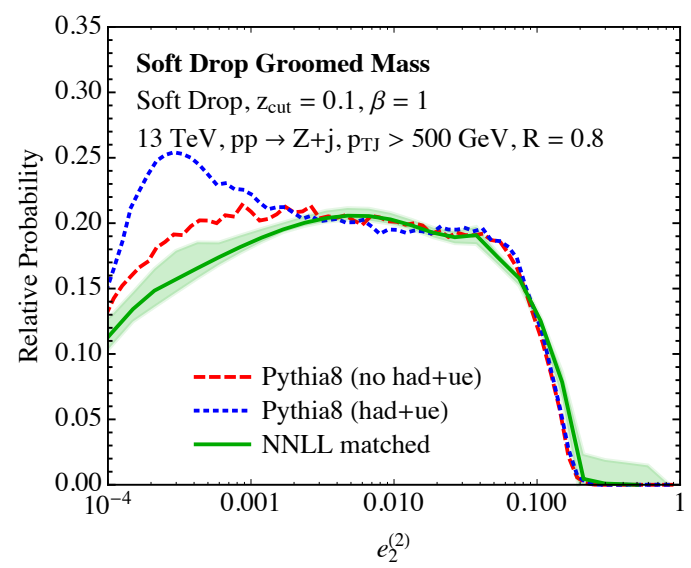

(d)

Figure 12. Comparison between soft-drop groomed $e_{2}^{(2)}$ distributions with $z_{\text {cut }}=0.1$ and $\beta=0$ (top) and $\beta=1$ (bottom) for matched and normalized NNLL, parton-level, and hadron-level Monte Carlo. All curves integrate to the same value over the range $e_{2}^{(2)} \in[0.001,0.1]$. The uncertainty band for soft drop with $\beta=1$ at NNLL includes the variation of the two-loop non-cusp anomalous dimension.

Figure 12 also illustrates that soft drop grooming eliminates sensitivity to both hadronization and underlying event until deep in the infrared. The parton-level and hadron-level distributions for each Monte Carlo agree almost perfectly until below about $e_{2}^{(2)} \lesssim 10^{-3}$. That hadronization effects are small is expected from our $e^{+} e^{-}$analysis, but this also demonstrates that underlying event effects are negligible. A similar observation was made in ref. [8], though at a much higher jet $p_{T}\left(p_{T}>3 \mathrm{TeV}\right)$. As in $e^{+} e^{-}$collisions, we expect that the hadronization effects that are observed in the Monte Carlo can be explained by a shape function, though we leave this to future work.

That the shape of the resummed distribution is both completely determined by collinear dynamics and is insensitive to underlying event suggests that by grooming jets with soft drop, we are able to completely isolate factorization-violating effects into an 


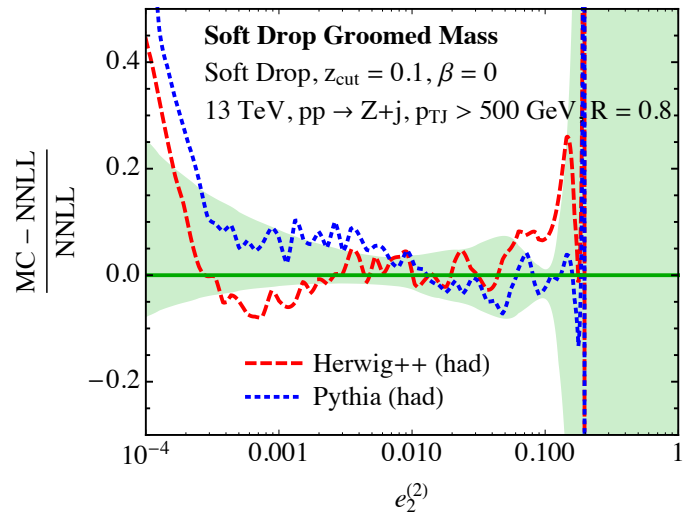

(a)

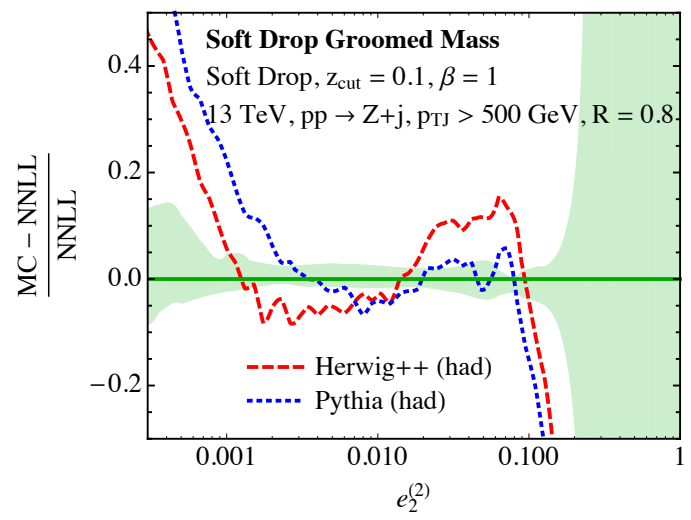

(b)

Figure 13. Direct comparison of hadron-level output from HeRwig ++ and PythiA already shown in figure 12. Soft drop is performed with $z_{\text {cut }}=0.1$ and both $\beta=0$ (left) and $\beta=1$ (right). Curves are displayed as relative differences between Monte Carlo output and our matched NNLL predictions, with theoretical uncertainties shown as a shaded band.

overall normalization. Therefore, we conjecture that the shape of the leading-power distribution of soft-drop groomed observables as measured in hadron collision events completely factorizes, just like the $p_{T}$ spectrum in Drell-Yan events [104]. We leave a proof of this conjecture to future work. ${ }^{9}$

\section{Conclusions}

In this paper, we presented the first calculation for an observable measured exclusively on the constituents of a jet to NNLL accuracy and matched to fixed-order results at $\mathcal{O}\left(\alpha_{s}^{2}\right)$ relative to the Born process. The ability to do this calculation required grooming the jet with the soft drop algorithm, which eliminates the complications due to non-global logarithms that afflict ungroomed jet measurements. The soft drop groomer also significantly reduces nonperturbative effects from hadronization and underlying event, rendering the perturbative calculation of energy correlation functions accurate over several decades. The insensitivity of soft-drop groomed jet observables to underlying event suggests that the normalized cross section fully factorizes in hadronic scattering events.

To complete the resummed calculation to NNLL accuracy required determining the two-loop non-cusp anomalous dimension for the soft function for which all emissions are removed. For $\beta=0$, we were able to use results from the literature to extract the non-cusp anomalous dimension, up to calculable clustering effects. While not used for results in this paper, the clustering effects when using the anti- $k_{T}$ algorithm with soft drop are closely

\footnotetext{
${ }^{9}$ Due to the presence of the complicated object $D_{k}\left(p_{T}^{\min }, \eta_{\max }, z_{\text {cut }}, R\right)$, eq. $(7.1)$ is not strictly a factorization theorem. It may not be possible to factorize $D_{k}$ to all orders due to the presence of so-called Glauber modes [104] in the cross section. While it is beyond the scope of this paper, recent work suggests that Glaubers can be included into the cross section directly [105], and our numerical work indicates that the effect may be absorbable into the normalization.
} 
related to similar effects found in jet veto calculations. For soft drop angular exponent $\beta>0$, we demonstrated a numerical procedure for determining the anomalous dimension using EVENT2. This was sufficient to approximate the non-cusp anomalous dimension, but a full calculation of the two-loop soft function for soft drop with $\beta \geq 0$ is desired.

With a complete calculation of the two-loop soft function, including constants, we would be one step closer to resumming to next-to-next-to-next-to-leading logarithmic accuracy $\left(\mathrm{N}^{3} \mathrm{LL}\right)$. Up to the unknown four-loop cusp anomalous dimension (whose effects have been shown to be small [106-108]), the only other piece to get to $\mathrm{N}^{3} \mathrm{LL}$ would be the three-loop non-cusp anomalous dimension of the soft-dropped soft function. Without an explicit three-loop calculation, this anomalous dimension could in principle be estimated using a technique similar to what we used at two loops, using a fixed-order code like EERAD3 [109]. If this is possible, then resummation to this accuracy would potentially reduce residual scale uncertainties to the percent-level, assuming a scaling of uncertainties like observed in going from NLL to NNLL.

For our complete predictions, it was vital to match our resummed calculations to high precision fixed-order distributions. Fixed-order calculations have been traditionally used for observables that are inclusive over soft and collinear radiation, like total cross sections or $p_{T}$ spectra. The generation of fixed-order differential distributions for the plots in this paper required CPU-centuries, which we attained only by running on thousands of cores. For calculations of more complicated jet observables, precise fixed order computations are likely infeasible with presently available tools. As jet substructure pushes to higher precision, it will be necessary to have fixed-order calculations that more efficiently sample the infrared regions of phase space.

The calculations in this paper represent a new frontier of precision QCD. While jet substructure techniques have been used for some time in experimental analyses at the LHC, they are just now approaching the level of theoretical precision that can be meaningfully compared to data. By soft-drop grooming jets, we greatly reduce the theoretical challenges, enabling the calculation of a wide range of jet substructure observables to full NNLL accuracy.

\section{Acknowledgments}

We thank Simone Marzani, Ian Moult, Ben Nachman, Duff Neill, Iain Stewart, and HuaXing Zhu for discussions. We thank Andrzej Siodmok for help with generation of events in Herwig++. A.L. is supported by the U.S. National Science Foundation, under grant PHY-1419008, the LHC Theory Initiative. The computations in this paper were run on the Odyssey cluster supported by the FAS Division of Science, Research Computing Group at Harvard University. 


\section{A Three-loop $\beta$-function and cusp anomalous dimension}

The $\beta$-function is defined to be

$$
\beta\left(\alpha_{s}\right)=\mu \frac{\partial \alpha_{s}}{\partial \mu}=-2 \alpha_{s} \sum_{n=0}^{\infty} \beta_{n}\left(\frac{\alpha_{s}}{4 \pi}\right)^{n+1}
$$

For NNLL resummation, we need the $\beta$-function to three-loop order $[71,72]$. The first three coefficients are

$$
\begin{aligned}
& \beta_{0}=\frac{11}{3} C_{A}-\frac{4}{3} T_{R} n_{f}, \\
& \beta_{1}=\frac{34}{3} C_{A}^{2}-4 T_{R} n_{f}\left(C_{F}+\frac{5}{3} C_{A}\right), \\
& \beta_{2}=\frac{2857}{54} C_{A}^{3}+T_{R} n_{f}\left(2 C_{F}^{2}-\frac{205}{9} C_{F} C_{A}-\frac{1415}{27} C_{A}^{2}\right)+T_{R}^{2} n_{f}^{2}\left(\frac{44}{9} C_{F}+\frac{158}{27} C_{A}\right) .
\end{aligned}
$$

For NNLL resummation, we need the cusp anomalous dimension

$$
\Gamma_{\text {cusp }}=\sum_{n=0}^{\infty} \Gamma_{n}\left(\frac{\alpha_{s}}{4 \pi}\right)^{n+1}
$$

to three-loop order. The first three coefficients of the cusp anomalous dimension are [67-70]:

$$
\begin{aligned}
& \Gamma_{0}=4 \\
& \Gamma_{1}=4 C_{A}\left(\frac{67}{9}-\frac{\pi^{2}}{3}\right)-\frac{80}{9} T_{R} n_{f}, \\
& \Gamma_{2}=4 C_{A}^{2}\left(\frac{245}{6}-\frac{134 \pi^{2}}{27}+\frac{11 \pi^{4}}{45}+\frac{22}{3} \zeta_{3}\right)+32 C_{A} T_{R} n_{f}\left(-\frac{209}{108}+\frac{5 \pi^{2}}{27}-\frac{7}{3} \zeta_{3}\right) \\
& \quad+4 C_{F} T_{R} n_{f}\left(16 \zeta_{3}-\frac{55}{3}\right)-\frac{64}{27} T_{R}^{2} n_{f}^{2} .
\end{aligned}
$$

\section{B Hard function}

The hard function for dijet production in $e^{+} e^{-}$collisions is defined by the Wilson coefficient for matching the full QCD current onto the SCET dijet operator. For $e^{+} e^{-} \rightarrow q \bar{q}$ events, the Wilson coefficient $C\left(Q^{2}, \mu\right)$ is

$$
\langle q \bar{q}|\bar{\psi} \Gamma \psi| 0\rangle=C\left(Q^{2}, \mu\right)\left\langle q \bar{q}\left|\bar{\chi}_{n} Y_{n}^{\dagger} \Gamma Y_{\bar{n}} \chi_{\bar{n}}\right| 0\right\rangle .
$$

Here, $\bar{\psi} \Gamma \psi$ is the QCD current for the production of a $q \bar{q}$ pair from the vacuum. $\chi_{\bar{n}}$ is a quark jet operator collinear quark operator defined in the light-like direction $\bar{n}$ in SCET. For calculations at leading power, $\chi_{n}=W_{t}^{\dagger} \psi$, with $W_{t}$ a Wilson line pointing in some direction $t$ not collinear to $n$ and $\psi$ is an ordinary quark field. The soft Wilson lines $Y_{n}$ and $Y_{\bar{n}}$ point in the $n$ and $\bar{n}$ directions respectively. $\Gamma$ represents a generic Dirac matrix. 
We have ignored contraction with the leptonic tensor for simplicity. The Wilson lines $Y_{n}$ is defined as

$$
Y_{n}\left(x^{\mu}\right)=\mathbf{P} \exp \left(i g \int_{0}^{\infty} d s n \cdot A\left(x^{\mu}+s n^{\mu}\right)\right),
$$

where $\mathbf{P}$ denotes path-ordering. $Y_{\bar{n}}$ and $W_{t}$ are defined similarly with $\bar{n}^{\mu}$ and $t^{\mu}$ replacing $n^{\mu}$. In SCET, the gluon fields in the Wilson line are soft gluons for the $Y$ 's and collinear gluons for the $W$ 's, but once the sectors are decoupled one can treat any of these gluons simply as a gluon field of full QCD.

The hard function is the square of the Wilson coefficient:

$$
H\left(Q^{2}, \mu\right)=\left|C\left(Q^{2}, \mu\right)\right|^{2}
$$

While we do not present its expression here, the hard function for $e^{+} e^{-} \rightarrow g g$ events is defined analogously, by matching the Higgs current $F_{\mu \nu} F^{\mu \nu}$ onto SCET.

\section{B.1 $e^{+} e^{-} \rightarrow q \bar{q}$}

The one-loop hard function for the process $e^{+} e^{-} \rightarrow q \bar{q}$ is $[23,39,110,111]$

$$
H=1+\frac{\alpha_{s} C_{F}}{2 \pi}\left(-L_{H}^{2}-3 L_{H}-8+\frac{7}{6} \pi^{2}\right)
$$

where

$$
L_{H}=\log \frac{\mu^{2}}{Q^{2}} .
$$

The cusp anomalous dimension of the hard function to all orders is

$$
\Gamma_{H}=-2 C_{F} \Gamma_{\text {cusp }}
$$

where $\Gamma_{\text {cusp }}$ is the cusp anomalous dimension defined in eq. (A.5). Similar to the cusp anomalous dimension, we define the coefficients of the non-cusp anomalous dimension $\gamma$ via

$$
\gamma=\sum_{n=0}^{\infty} \gamma^{(n)}\left(\frac{\alpha_{s}}{4 \pi}\right)^{n+1}
$$

Through two-loops, the non-cusp anomalous dimension coefficients of the hard function are $[73,74]$

$$
\begin{aligned}
& \gamma_{H}^{(0)}=-12 C_{F}, \\
& \gamma_{H}^{(1)}=\left(-6+8 \pi^{2}-96 \zeta_{3}\right) C_{F}^{2}+\left(-\frac{1922}{27}-\frac{22}{3} \pi^{2}+104 \zeta_{3}\right) C_{F} C_{A}+\left(\frac{520}{27}+\frac{8}{3} \pi^{2}\right) C_{F} n_{f} T_{R} .
\end{aligned}
$$

\section{B.2 $e^{+} e^{-} \rightarrow g g$}

In the infinite top quark mass limit or with a finite Yukawa coupling, $e^{+} e^{-}$scattering can produce final state gluon jets. The hard function for such a process can be extracted from 
$g g \rightarrow H$ calculations. To all orders, the cusp anomalous dimension of the $e^{+} e^{-} \rightarrow g g$ hard function is

$$
\Gamma_{H}=-2 C_{A} \Gamma_{\text {cusp }},
$$

where $\Gamma_{\text {cusp }}$ is the cusp anomalous dimension in eq. (A.5). Through two-loops, the coefficients of the non-cusp anomalous dimension are [112-114]

$$
\begin{aligned}
& \gamma_{H}^{(0)}=-4 \beta_{0}, \\
& \gamma_{H}^{(1)}=\left(-\frac{236}{9}+8 \zeta_{3}\right) C_{A}^{2}+\left(-\frac{76}{9}+\frac{2}{3} \pi^{2}\right) C_{A} \beta_{0}-4 \beta_{1} .
\end{aligned}
$$

\section{The global soft function}

For arbitrary exponent $\beta$ in the soft-drop groomer, the soft function can be calculated by requiring that soft gluons in measured jets fail the soft drop criterion. For hemisphere jets in $e^{+} e^{-} \rightarrow q \bar{q}$ events, for example, the soft function is defined by the forward matrix element of soft Wilson lines:

$$
S_{\mathrm{G}}\left(z_{\text {cut }}\right)=\frac{1}{N_{C}} \operatorname{tr}\left\langle 0\left|\mathrm{~T}\left\{Y_{n} Y_{\bar{n}}\right\} \hat{\Theta}_{S D} \overline{\mathrm{T}}\left\{Y_{n} Y_{\bar{n}}\right\}\right| 0\right\rangle .
$$

Here, $n$ and $\bar{n}$ are the light-like directions of the $q \bar{q}$ dipole, T denotes time ordering, and $\hat{\Theta}_{S D}$ denotes the soft drop groomer operator which requires the final state to fail soft drop. The action of $\hat{\Theta}_{S D}$ on soft final states cannot be written in a closed form for an arbitrary final state due to clustering effects, though it can be defined order-by-order. For example, the matrix element of $\hat{\Theta}_{S D}$ for $\beta=0$ on a final state with two soft particles was presented in section 5.1.1.

At one-loop for hemisphere jets in $e^{+} e^{-}$collisions, the soft function $S_{\mathrm{G}}$ can be calculated from

$$
\begin{aligned}
S_{\mathrm{G}}=g^{2} \mu^{2 \epsilon} C_{i} \int & \frac{d^{d} k}{(2 \pi)^{d}} \frac{n \cdot \bar{n}}{n \cdot k k \cdot \bar{n}} 2 \pi \delta\left(k^{2}\right) \Theta\left(k^{0}\right) \Theta(\bar{n} \cdot k-n \cdot k) \Theta\left(z_{\text {cut }} \frac{Q}{2}\left[2 \frac{n \cdot k}{k^{0}}\right]^{\beta / 2}-k^{0}\right) \\
+(n \leftrightarrow \bar{n}), & \text { (C.2) }
\end{aligned}
$$

where $n, \bar{n}$ are back-to-back light-like vectors with $n \cdot \bar{n}=2$. The requirement $\bar{n} \cdot k>n \cdot k$ restricts the radiation to lie in one hemisphere, while the requirement

$$
z_{\text {cut }} \frac{Q}{2}\left[2 \frac{n \cdot k}{k^{0}}\right]^{\beta / 2}>k^{0}
$$

restricts the soft gluon to fail soft drop. We find

$$
S_{\mathrm{G}}=1+\frac{\alpha_{s} C_{i}}{\pi}\left[\frac{1}{2(1+\beta)} L_{S}^{2}-\frac{\pi^{2}}{12}\left(\frac{1}{1+\beta}+2+\beta\right)\right],
$$

where $C_{i}$ is the appropriate color factor $\left(C_{F}\right.$ for $e^{+} e^{-} \rightarrow q \bar{q} ; C_{A}$ for $\left.e^{+} e^{-} \rightarrow g g\right)$ and

$$
L_{S}=\log \frac{\mu^{2}}{Q^{2}\left(z_{\text {cut }}\right)^{2} 4^{\beta}} .
$$


To all orders, the cusp anomalous dimension of the hemisphere wide-angle soft function is

$$
\Gamma_{S}=\frac{2 C_{i}}{1+\beta} \Gamma_{\text {cusp }}
$$

where $\Gamma_{\text {cusp }}$ is the cusp anomalous dimension from eq. (A.5). To one-loop order, the non-cusp anomalous dimension is 0 :

$$
\gamma_{S}^{(0)}=0 .
$$

For NNLL resummation, we need the non-cusp anomalous dimension to two-loop order. As discussed in section 5.1.1, for soft drop with angular exponent $\beta=0$, this can be extracted from energy veto calculations, up to clustering effects that we calculated. For soft drop with $\beta=0$ and Cambridge/Aachen reclustering, we find the two-loop non-cusp anomalous dimension to be

$$
\left.\gamma_{S}^{(1)}\right|_{\beta=0}=C_{i}\left[34.01 C_{F}+\left(\frac{1616}{27}-56 \zeta_{3}-9.31\right) C_{A}-\left(\frac{448}{27}+14.04\right) n_{f} T_{R}-\frac{2 \pi^{2}}{3} \beta_{0}\right] .
$$

\section{Jet functions}

Here, we present the quark and gluon jet functions on which the energy correlation function $e_{2}^{(\alpha)}$ is measured. The quark jet function, for example, is defined by the forward matrix element:

$$
J_{q}\left(e_{2}^{(\alpha)}\right)=\frac{(2 \pi)^{3}}{N_{C}} \operatorname{tr}\left\langle 0\left|\frac{\hbar}{2} \chi_{n}(0) \delta(Q-\bar{n} \cdot \mathcal{P}) \delta^{(2)}\left(\overrightarrow{\mathcal{P}}_{\perp}\right) \delta\left(e_{2}^{(\alpha)}-\hat{e}_{2}^{(\alpha)}\right) \bar{\chi}_{n}(0)\right| 0\right\rangle .
$$

Here, the jet is collinear to the light-like direction $n$, the operator $\delta(Q-\bar{n} \cdot \mathcal{P})$ restricts the large light-cone component of momentum to be equal to the center-of-mass collision energy $Q$, and $\delta^{(2)}\left(\overrightarrow{\mathcal{P}}_{\perp}\right)$ restricts the jet function to have zero net momentum transverse to the $n$ direction. The measurement operator is defined by its action on an $n$-particle collinear final state $\left|X_{n}\right\rangle$ as:

$$
\hat{e}_{2}^{(\alpha)}\left|X_{n}\right\rangle=\frac{2^{3 \alpha / 2}}{Q^{2}} \sum_{i<j \in X_{n}}\left(\bar{n} \cdot p_{i}\right)^{1-\alpha / 2}\left(\bar{n} \cdot p_{j}\right)^{1-\alpha / 2}\left(p_{i} \cdot p_{j}\right)^{\alpha / 2}\left|X_{n}\right\rangle .
$$

To write this expression, we have expanded the definition of the energy correlation function from section 2.2 to leading power with collinear momenta. The gluon jet function is defined similarly:

$$
J_{g}\left(e_{2}^{(\alpha)}\right)=\frac{(2 \pi)^{3}}{N_{C}} \operatorname{tr}\left\langle 0\left|\mathcal{B}_{\perp}^{\mu}(0) \delta(Q-\bar{n} \cdot \mathcal{P}) \delta^{(2)}\left(\overrightarrow{\mathcal{P}}_{\perp}\right) \delta\left(e_{2}^{(\alpha)}-\hat{e}_{2}^{(\alpha)}\right) \mathcal{B}_{\perp \mu}(0)\right| 0\right\rangle,
$$

where $\mathcal{B}_{\perp}^{\mu}$ is the collinear-gauge invariant operator in SCET that creates physical collinear gluons.

The following expressions will be presented in Laplace space, where renormalization is multiplicative and the Laplace space conjugate is $\nu$. That is,

$$
J(\nu)=\int_{0}^{\infty} d e_{2}^{(\alpha)} e^{-\nu e_{2}^{(\alpha)}} J\left(e_{2}^{(\alpha)}\right) .
$$

The one-loop quark and gluon jet functions were first calculated in ref. [25] for jets on which the two-point energy correlation functions with arbitrary angular exponent are measured. 


\section{D.1 Quark jets}

To one loop, the Laplace-space quark jet function is

$$
J_{q}(\nu)=1+\frac{\alpha_{s} C_{F}}{2 \pi}\left[\frac{\alpha}{2(\alpha-1)} L_{C}^{2}+\frac{3}{2} L_{C}+\left(\frac{13}{2}-\frac{12}{2 \alpha}\right)-\frac{\pi^{2}}{12}\left(9-\frac{3}{\alpha-1}-\frac{4}{\alpha}\right)\right],
$$

where

$$
L_{C}=\log \frac{\mu^{2}\left(\nu e^{\gamma_{E}}\right)^{2 / \alpha}}{E_{J}^{2}} .
$$

To all orders, the cusp anomalous dimension of the quark jet function is

$$
\Gamma_{C}^{q}=\frac{\alpha}{\alpha-1} C_{F} \Gamma_{\text {cusp }},
$$

where $\Gamma_{\text {cusp }}$ is the cusp anomalous dimension from eq. (A.5). For all $\alpha$, the one-loop non-cusp anomalous dimension is

$$
\gamma_{C}^{q,(0)}=6 C_{F} .
$$

For NNLL resummation, we also need the two-loop non-cusp anomalous dimension. For $\alpha=2$, corresponding to jet mass or thrust, this is known exactly. In that case, the non-cusp anomalous dimension is [79]

$$
\left.\gamma_{C}^{q,(1)}\right|_{\alpha=2}=C_{F}\left[C_{F}\left(3-4 \pi^{2}+48 \zeta_{3}\right)+C_{A}\left(\frac{1769}{27}+\frac{22 \pi^{2}}{9}-80 \zeta_{3}\right)+T_{R} n_{f}\left(-\frac{484}{27}-\frac{8 \pi^{2}}{9}\right)\right] .
$$

\section{D.2 Gluon jets}

To one-loop, the Laplace-space gluon jet function is

$$
J_{g}(\nu)=1+\frac{\alpha_{s}}{2 \pi}\left[\frac{\alpha C_{A}}{2(\alpha-1)} L_{C}^{2}+\frac{\beta_{0}}{2} L_{C}+C_{A}\left(\frac{67}{9} \frac{\alpha-1}{\alpha}-\frac{\pi^{2}}{3} \frac{2(\alpha-1)^{2}-1}{\alpha-1}\right)+n_{f} T_{R}\left(\frac{26}{9 \alpha}-\frac{23}{9}\right)\right],
$$

where

$$
L_{C}=\log \frac{\mu^{2}\left(\nu e^{\gamma_{E}}\right)^{2 / \alpha}}{E_{J}^{2}} .
$$

To all orders, the cusp anomalous dimension of the gluon jet function is

$$
\Gamma_{C}^{g}=\frac{\alpha}{\alpha-1} C_{A} \Gamma_{\mathrm{cusp}},
$$

where $\Gamma_{\text {cusp }}$ is the cusp anomalous dimension from eq. (A.5). For all $\alpha$, the one-loop non-cusp anomalous dimension is

$$
\gamma_{C}^{g,(0)}=2 \beta_{0}
$$

For NNLL resummation, we also need the two-loop non-cusp anomalous dimension. For $\alpha=2$, corresponding to jet mass or thrust, this is known exactly. In that case, the non-cusp anomalous dimension is [115]

$$
\gamma_{C}^{g,(1)}=C_{A}^{2}\left(\frac{2192}{27}-\frac{22 \pi^{2}}{9}-32 \zeta_{3}\right)+C_{A} T_{R} n_{f}\left(-\frac{736}{27}+\frac{8 \pi^{2}}{9}\right)-8 C_{F} T_{R} n_{f} .
$$




\section{E Collinear-soft function}

The final piece in the factorization theorem is the collinear soft function, defined from soft radiation that is collinear to the jet. As it describes soft radiation, the collinear-soft function is defined as a forward matrix element of Wilson lines:

$$
S_{C}\left(z_{\mathrm{cut}} e_{2}^{(\alpha)}\right)=\frac{1}{N_{C}} \operatorname{tr}\left\langle 0\left|\mathrm{~T}\left\{Y_{n}^{\dagger} W_{t}\right\} \delta\left(e_{2}^{(\alpha)}-\left(1-\hat{\Theta}_{S D}\right) \hat{e}_{2}^{(\alpha)}\right) \overline{\mathrm{T}}\left\{W_{t}^{\dagger} Y_{n}\right\}\right| 0\right\rangle .
$$

The $Y$ and $W$ Wilson lines are the same as the ones in the soft and jet functions respectively, but depend on collinear-soft fields (which, like any of the others, can be treated as full QCD fields at leading power).

Now, collinear-soft modes only contribute to $e_{2}^{(\alpha)}$ if emissions pass the soft drop groomer: this is denoted by $1-\hat{\Theta}_{S D}$ in the measurement function. (Recall that $\hat{\Theta}_{S D}$ removes emissions from the jet according to soft drop.) Again, this operator cannot be written in closed form for an arbitrary final state due to clustering effects, but below, we will calculate it explicitly at one-loop. The $\hat{e}_{2}^{(\alpha)}$ measurement operator is defined by its action on an $n$-particle collinear-soft final state $\left|X_{S, n}\right\rangle$ :

$$
\hat{e}_{2}^{(\alpha)}\left|X_{S, n}\right\rangle=\frac{2^{\alpha}}{Q} \sum_{i \in X_{S, n}}\left(\bar{n} \cdot p_{i}\right)^{1-\alpha / 2}\left(n \cdot p_{i}\right)^{\alpha / 2}\left|X_{S, n}\right\rangle .
$$

This follows from expanding the definition of the energy correlation function from section 2.2 to leading power with collinear-soft momenta.

This can be calculated at one-loop accuracy from

$$
\begin{array}{r}
S_{C}=g^{2} \mu^{2 \epsilon} C_{i} \int \frac{d^{d} k}{(2 \pi)^{d}} \frac{n \cdot \bar{n}}{n \cdot k k \cdot \bar{n}} 2 \pi \delta\left(k^{2}\right) \Theta(\bar{n} \cdot k)\left[\Theta\left(z_{\text {cut }}\left[4 \frac{n \cdot k}{\bar{n} \cdot k}\right]^{\beta / 2}-\frac{\bar{n} \cdot k}{Q}\right) \delta\left(e_{2}^{(\alpha)}\right)\right. \\
\left.+\Theta\left(\frac{\bar{n} \cdot k}{Q}-z_{\text {cut }}\left[4 \frac{n \cdot k}{\bar{n} \cdot k}\right]^{\beta / 2}\right) \delta\left(e_{2}^{(\alpha)}-\frac{2^{\alpha}}{Q}(n \cdot k)^{\alpha / 2}(\bar{n} \cdot k)^{1-\alpha / 2}\right)\right] \\
=g^{2} \mu^{2 \epsilon} C_{i} \int \frac{d^{d} k}{(2 \pi)^{d}} \frac{n \cdot \bar{n}}{n \cdot k k \cdot \bar{n}} 2 \pi \delta\left(k^{2}\right) \Theta(\bar{n} \cdot k) \Theta\left(\frac{\bar{n} \cdot k}{Q}-z_{\text {cut }}\left[4 \frac{n \cdot k}{\bar{n} \cdot k}\right]^{\beta / 2}\right) \\
\times\left[\delta\left(e_{2}^{(\alpha)}-\frac{2^{\alpha}}{Q}(n \cdot k)^{\alpha / 2}(\bar{n} \cdot k)^{1-\alpha / 2}\right)-\delta\left(e_{2}^{(\alpha)}\right)\right] \cdot
\end{array}
$$

where $C_{i}$ is the color factor of the jet. In the second equality, we have rearranged the phase space constraints and explicitly removed scaleless integrals. For this collinear-soft function, at one-loop in Laplace space we find

$$
S_{C}(\nu)=1+\frac{\alpha_{s} C_{i}}{2 \pi}\left[-\frac{\alpha+\beta}{2(\alpha-1)(\beta+1)} L_{S_{C}}^{2}+\frac{\pi^{2}}{12} \frac{(\alpha+2+3 \beta)(\alpha-2-\beta)}{(\alpha+\beta)(\alpha-1)(\beta+1)}\right],
$$

where

$$
L_{S_{C}}=\log \frac{\mu^{2}\left(\nu e^{\gamma_{E}}\right)^{2 \frac{\beta+1}{\alpha+\beta}}}{E_{J}^{2}\left(z_{\text {cut }}\right)^{2 \frac{\alpha-1}{\alpha+\beta}}}
$$


To all orders, the cusp anomalous dimension of the collinear-soft function is

$$
\Gamma_{S_{C}}=-C_{i} \frac{\alpha+\beta}{(\alpha-1)(\beta+1)} \Gamma_{\text {cusp }}
$$

where $\Gamma_{\text {cusp }}$ is the cusp anomalous dimension from eq. (A.5). To one-loop order, the noncusp anomalous dimension is 0 :

$$
\gamma_{S_{C}}^{(0)}=0
$$

For NNLL resummation, we need the non-cusp anomalous dimension to two-loop order. For $\alpha=2$ and $\beta=0$, this can be determined by renormalization group consistency of the cross section directly, using either the $e^{+} e^{-} \rightarrow q \bar{q}$ or the $e^{+} e^{-} \rightarrow g g$ process. For soft drop with Cambridge/Aachen reclustering, the two-loop non-cusp anomalous dimension is

$$
\left.\gamma_{S_{C}}^{(1)}\right|_{\alpha=2, \beta=0}=C_{i}\left[-17.00 C_{F}+\left(-55.20+\frac{22 \pi^{2}}{9}+56 \zeta_{3}\right) C_{A}+\left(23.61-\frac{8 \pi^{2}}{9}\right) n_{f} T_{R}\right] .
$$

\section{F Resummation}

Because we work in Laplace space, defined according to

$$
F(\nu)=\int_{0^{-}}^{\infty} d e_{2}^{(\alpha)} e^{-\nu e_{2}^{(\alpha)}} F\left(e_{2}^{(\alpha)}\right),
$$

the renormalization of all functions in the factorization theorem is multiplicative. For some function $F$ in the factorization theorem, it generically has the renormalization equation

$$
\mu \frac{\partial}{\partial \mu} F(\mu)=\gamma F(\mu)
$$

where the anomalous dimension of $F$ is $\gamma$. The anomalous dimension can be written as

$$
\gamma=\Gamma_{F}\left(\alpha_{s}\right) \log \frac{\mu^{2}}{\mu_{1}^{2}}+\gamma_{F}\left(\alpha_{s}\right)
$$

where $\Gamma_{F}\left(\alpha_{s}\right)$ is the cusp part of the anomalous dimension, $\mu_{1}$ is the infrared scale in the logarithm and $\gamma_{F}\left(\alpha_{s}\right)$ is the non-cusp part of the anomalous dimension. The solution ${ }^{10}$ to the renormalization group equation can be written more conveniently as an integral with respect to $\alpha_{s}$, by using the definition of the $\beta$-function as

$$
\frac{d \mu}{\mu}=\frac{d \alpha_{s}}{\beta\left(\alpha_{s}\right)} .
$$

Then, the solution to eq. (F.3) can be expressed as

$$
\begin{aligned}
F(\mu)=F\left(\mu_{0}\right) \exp \left[2 \int_{\alpha_{s}\left(\mu_{0}\right)}^{\alpha_{s}(\mu)} \frac{d \alpha}{\beta(\alpha)} \Gamma_{F}(\alpha) \int_{\alpha_{s}\left(\mu_{0}\right)}^{\alpha} \frac{d \alpha^{\prime}}{\beta\left(\alpha^{\prime}\right)}\right. & +\int_{\alpha_{s}\left(\mu_{0}\right)}^{\alpha_{s}(\mu)} \frac{d \alpha}{\beta(\alpha)} \gamma_{F}(\alpha) \\
& \left.+\log \frac{\mu_{0}^{2}}{\mu_{1}^{2}} \int_{\alpha_{s}\left(\mu_{0}\right)}^{\alpha_{s}(\mu)} \frac{d \alpha}{\beta(\alpha)} \Gamma_{F}(\alpha)\right]
\end{aligned}
$$

\footnotetext{
${ }^{10}$ In the plots of resummed distributions in this paper, we have frozen the strong coupling at $\mu_{\mathrm{NP}}=1 \mathrm{GeV}$ to keep cross sections finite. In the case of frozen $\alpha_{s}$, the solution to the renormalization group equation for each $F(\mu)$ is quite simple, so we omit the details of the prescription below $\mu_{\mathrm{NP}}$ here.
} 
where $\mu_{0}$ is a reference scale.

The exponentiated kernels can be explicitly evaluated to any logarithmic accuracy given the anomalous dimensions. The cusp-part of the anomalous dimension, $\Gamma_{F}\left(\alpha_{s}\right)$, is proportional to the cusp anomalous dimension, $\Gamma_{F}\left(\alpha_{s}\right)=d_{F} \Gamma_{\text {cusp }}$, where $d_{F}$ includes an appropriate color factor. The cusp anomalous dimension has an expansion in $\alpha_{s}$ given by eq. (A.5). The non-cusp anomalous dimension has a similar expansion defined in eq. (B.7). For resummation to NNLL accuracy, we need the $\gamma_{0}$ and $\gamma_{1}$ coefficients, corresponding to computing the anomalous dimensions of the functions in the factorization theorem to two-loops.

With these expansions, we are able to explicitly evaluate the exponentiated kernel to NNLL accuracy. We have:

$$
\begin{gathered}
K_{F}\left(\mu, \mu_{0}\right) \equiv 2 \int_{\alpha_{s}\left(\mu_{0}\right)}^{\alpha_{s}(\mu)} \frac{d \alpha}{\beta(\alpha)} \Gamma_{F}(\alpha) \int_{\alpha_{s}\left(\mu_{0}\right)}^{\alpha} \frac{d \alpha^{\prime}}{\beta\left(\alpha^{\prime}\right)}+\int_{\alpha_{s}\left(\mu_{0}\right)}^{\alpha_{s}(\mu)} \frac{d \alpha}{\beta(\alpha)} \gamma_{F}(\alpha) \\
=C_{i} \frac{\Gamma_{0}}{2 \beta_{0}^{2}}\left\{\frac{4 \pi}{\alpha_{s}\left(\mu_{0}\right)}\left(\log r+\frac{1}{r}-1\right)+\left(\frac{\Gamma_{1}}{\Gamma_{0}}-\frac{\beta_{1}}{\beta_{0}}\right)(r-1-\log r)-\frac{\beta_{1}}{2 \beta_{0}} \log ^{2} r\right. \\
+\frac{\alpha_{s}\left(\mu_{0}\right)}{4 \pi}\left[\left(\frac{\Gamma_{1} \beta_{1}}{\Gamma_{0} \beta_{0}}-\frac{\beta_{1}^{2}}{\beta_{0}^{2}}\right)(r-1-r \log r)-\left(\frac{\beta_{1}^{2}}{\beta_{0}^{2}}-\frac{\beta_{2}}{\beta_{0}}\right) \log r\right. \\
\left.\left.\quad+\left(\frac{\Gamma_{2}}{\Gamma_{0}}-\frac{\Gamma_{1} \beta_{1}}{\Gamma_{0} \beta_{0}}+\frac{\beta_{1}^{2}}{\beta_{0}^{2}}-\frac{\beta_{2}}{\beta_{0}}\right) \frac{r^{2}-1}{2}+\left(\frac{\Gamma_{2}}{\Gamma_{0}}-\frac{\Gamma_{1} \beta_{1}}{\Gamma_{0} \beta_{0}}\right)(1-r)\right]\right\} \\
-\frac{\gamma_{0}}{2 \beta_{0}} \log r-\frac{\gamma_{0}}{2 \beta_{0}} \frac{\alpha_{s}\left(\mu_{0}\right)}{4 \pi}\left(\frac{\gamma_{1}}{\gamma_{0}}-\frac{\beta_{1}}{\beta_{0}}\right)(r-1),
\end{gathered}
$$

where

$$
r=\frac{\alpha_{s}(\mu)}{\alpha_{s}\left(\mu_{0}\right)}
$$

The other exponentiated factor is

$$
\begin{aligned}
& \omega_{F}\left(\mu, \mu_{0}\right) \equiv \int_{\alpha_{s}\left(\mu_{0}\right)}^{\alpha_{s}(\mu)} \frac{d \alpha}{\beta(\alpha)} \Gamma_{F}(\alpha) \\
&=-C_{i} \frac{\Gamma_{0}}{2 \beta_{0}}\left\{\log r+\frac{\alpha_{s}\left(\mu_{0}\right)}{4 \pi}\left(\frac{\Gamma_{1}}{\Gamma_{0}}-\frac{\beta_{1}}{\beta_{0}}\right)(r-1)\right. \\
&\left.\quad+\frac{1}{2} \frac{\alpha_{s}^{2}\left(\mu_{0}\right)}{(4 \pi)^{2}}\left(\frac{\beta_{1}^{2}}{\beta_{0}^{2}}-\frac{\beta_{2}}{\beta_{0}}+\frac{\Gamma_{2}}{\Gamma_{0}}-\frac{\Gamma_{1} \beta_{1}}{\Gamma_{0} \beta_{0}}\right)\left(r^{2}-1\right)\right\} .
\end{aligned}
$$

Then, we can write the solution in Laplace space to the renormalization group equation in eq. (F.5) as

$$
F(\mu)=e^{K_{F}\left(\mu, \mu_{0}\right)} F\left(\mu_{0}\right)\left(\frac{\mu_{0}^{2}}{\mu_{1}^{2}}\right)^{\omega_{F}\left(\mu, \mu_{0}\right)} .
$$

Because the hard function and the wide-angle soft function are independent of the observable $e_{2}^{(\alpha)}$, their renormalization group equations are identical in real space and Laplace conjugate space. For the jet functions and the collinear-soft function, the inverse Laplace transform is non-trivial. 
For any of the jet functions appearing in the factorization theorem, the Laplace space solution can be written as

$$
J(\nu, \mu)=e^{K_{J}\left(\mu, \mu_{0}\right)} J\left(\nu, \mu_{0}\right)\left[\frac{\mu_{0}^{2}}{E_{J}^{2}}\left(\nu e^{\gamma_{E}}\right)^{2 / \alpha}\right]^{\omega_{J}\left(\mu, \mu_{0}\right)} .
$$

Note that the logarithms that appear in the low-scale jet function $J\left(\nu, \mu_{0}\right)$ have the same argument as the factor that is raised to the $\omega_{J}$ power. Therefore, using the relationship (noted by ref. [80])

$$
\frac{\partial^{n}}{\partial q^{n}} \nu^{q}=\nu^{q} \log ^{n} \nu
$$

we can re-write the jet function as

$$
J(\nu, \mu)=e^{K_{J}\left(\mu, \mu_{0}\right)} J\left(L \rightarrow \partial_{\omega_{J}}\right)\left[\frac{\mu_{0}^{2}}{E_{J}^{2}}\left(\nu e^{\gamma_{E}}\right)^{2 / \alpha}\right]^{\omega_{J}\left(\mu, \mu_{0}\right)} .
$$

Here $J\left(L \rightarrow \partial_{\omega_{J}}\right)$ means that the logarithms in the low-scale jet function $J\left(\nu, \mu_{0}\right)$ are replaced by derivatives with respect to the exponentiated factor $\omega_{J}\left(\mu, \mu_{0}\right)$. The exact same replacement can be made for the collinear-soft function. In that case, we have

$$
S_{C}\left(\nu, z_{\text {cut }}, \mu\right)=e^{K_{S_{C}}\left(\mu, \mu_{0}\right)} S_{C}\left(L \rightarrow \partial_{\omega_{S_{C}}}\right)\left[\frac{\mu_{0}^{2}\left(\nu e^{\gamma_{E}}\right)^{2 \frac{\beta+1}{\alpha+\beta}}}{E_{J}^{2}\left(z_{\text {cut }}\right)^{2 \frac{\alpha-1}{\alpha+\beta}}}\right]^{\omega_{S_{C}}\left(\mu, \mu_{0}\right)} .
$$

This re-writing of the jet and collinear-soft functions allows for very straightforward inverse Laplace transformation. In Laplace space, the total differential cross section for left and right hemisphere jets in $e^{+} e^{-}$collisions is

$$
\begin{aligned}
& \sigma(\nu) \\
& =\exp \left[K_{H}\left(\mu, \mu_{H}\right)+K_{S}\left(\mu, \mu_{S}\right)+K_{S_{C}}\left(\mu, \mu_{S_{C}}^{(L)}\right)+K_{S_{C}}\left(\mu, \mu_{S_{C}}^{(R)}\right)+K_{J}\left(\mu, \mu_{J}^{(L)}\right)+K_{J}\left(\mu, \mu_{J}^{(R)}\right)\right] \\
& \times H\left(Q, \mu_{H}\right) S\left(z_{\mathrm{cut}}, \mu_{S}\right) S_{C}\left(L \rightarrow \partial_{\omega_{S_{C}}}^{(L)}\right) S_{C}\left(L \rightarrow \partial_{\omega_{S_{C}}}^{(R)}\right) J\left(L \rightarrow \partial_{\omega_{J}}^{(L)}\right) J\left(L \rightarrow \partial_{\omega_{J}}^{(R)}\right) \\
& \times\left(\frac{\mu_{H}^{2}}{Q^{2}}\right)^{\omega_{H}\left(\mu, \mu_{H}\right)}\left(\frac{\mu_{S}^{2}}{4^{\beta} z_{\mathrm{cut}}^{2} Q^{2}}\right)^{\omega_{S}\left(\mu, \mu_{S}\right)}\left[\frac{\left(\mu_{S_{C}}^{(L)}\right)^{2}\left(\nu e^{\gamma_{E}}\right)^{\frac{\beta+1}{\alpha+\beta}}}{E_{J}^{2}\left(z_{\mathrm{cut}}\right)^{\frac{\alpha-1}{\alpha+\beta}}}\right]^{\omega_{S_{C}}\left(\mu, \mu_{S_{C}}^{(L)}\right)} \\
& \times\left[\frac{\left(\mu_{S_{C}}^{(R)}\right)^{2}\left(\nu e^{\gamma_{E}}\right)^{2 \frac{\beta+1}{\alpha+\beta}}}{E_{J}^{2}\left(z_{\mathrm{cut}}\right)^{2 \frac{\alpha-1}{\alpha+\beta}}}\right]^{\omega_{S_{C}}\left(\mu, \mu_{S_{C}}^{(R)}\right)}\left[\frac{\left(\mu_{J}^{(L)}\right)^{2}}{E_{J}^{2}}\left(\nu e^{\gamma_{E}}\right)^{2 / \alpha}\right]^{\omega_{J}\left(\mu, \mu_{J}^{(L)}\right)}\left[\frac{\left(\mu_{J}^{(R)}\right)^{2}}{E_{J}^{2}}\left(\nu e^{\gamma_{E}}\right)^{2 / \alpha}\right]^{\omega_{J}^{\left(\mu, \mu_{J}^{(R)}\right)} .}
\end{aligned}
$$

Note that the inverse Laplace transform commutes with the derivatives, and we have

$$
\mathcal{L}^{-1}\left[\nu^{q}\right]=\frac{\left(e_{2}^{(\alpha)}\right)^{-q-1}}{\Gamma(-q)} .
$$

Therefore, the differential cross section in real space can be written as:

$$
\begin{aligned}
& e_{2, L}^{(\alpha)} e_{2, R}^{(\alpha)} \frac{d^{2} \sigma}{d e_{2, L}^{(\alpha)} d e_{2, R}^{(\alpha)}} \\
& =\exp \left[K_{H}\left(\mu, \mu_{H}\right)+K_{S}\left(\mu, \mu_{S}\right)+K_{S_{C}}\left(\mu, \mu_{S_{C}}^{(L)}\right)+K_{S_{C}}\left(\mu, \mu_{S_{C}}^{(R)}\right)+K_{J}\left(\mu, \mu_{J}^{(L)}\right)+K_{J}\left(\mu, \mu_{J}^{(R)}\right)\right]
\end{aligned}
$$




$$
\begin{aligned}
& \times H\left(Q, \mu_{H}\right) S\left(z_{\mathrm{cut}}, \mu_{S}\right) S_{C}\left(L \rightarrow \partial_{\omega_{S_{C}}}^{(L)}\right) S_{C}\left(L \rightarrow \partial_{\omega_{S_{C}}}^{(R)}\right) J\left(L \rightarrow \partial_{\omega_{J}}^{(L)}\right) J\left(L \rightarrow \partial_{\omega_{J}}^{(R)}\right) \\
& \times\left(\frac{\mu_{H}^{2}}{Q^{2}}\right)^{\omega_{H}\left(\mu, \mu_{H}\right)}\left(\frac{\mu_{S}^{2}}{4^{\beta} z_{\mathrm{cut}}^{2} Q^{2}}\right)^{\omega_{S}\left(\mu, \mu_{S}\right)}\left[\frac{\left(\mu_{S_{C}}^{(L)}\right)^{2}\left(e_{2, L}^{(\alpha)} e^{-\gamma_{E}}\right)^{-2 \frac{\beta+1}{\alpha+\beta}}}{E_{J}^{2}\left(z_{\mathrm{cut}}\right)^{2 \frac{\alpha-1}{\alpha+\beta}}}\right]^{\omega_{S_{C}}\left(\mu, \mu_{S_{C}}^{(L)}\right)} \\
& \times\left[\frac{\left(\mu_{S_{C}}^{(R)}\right)^{2}\left(e_{2, R}^{(\alpha)} e^{-\gamma_{E}}\right)^{-2 \frac{\beta+1}{\alpha+\beta}}}{E_{J}^{2}\left(z_{\mathrm{cut}}\right)^{2 \frac{\alpha-1}{\alpha+\beta}}}\right]^{\omega_{S_{C}}\left(\mu, \mu_{S_{C}}^{(R)}\right)}\left[\frac{\left(\mu_{J}^{(L)}\right)^{2}}{E_{J}^{2}}\left(\frac{e^{\gamma_{E}}}{e_{2, L}^{(\alpha)}}\right)^{2 / \alpha}\right]^{\omega_{J}^{\left(\mu, \mu_{J}^{(L)}\right)}}\left[\frac{\left(\mu_{J}^{(R)}\right)^{2}}{E_{J}^{2}}\left(\frac{e^{\gamma_{E}}}{e_{2, R}^{(\alpha)}}\right)^{2 / \alpha}\right]^{\omega_{J}\left(\mu, \mu_{J}^{(R)}\right)} \\
& \times\left[\Gamma\left(-\frac{2(\beta+1)}{\alpha+\beta} \omega_{S_{C}}\left(\mu, \mu_{S_{C}}^{(L)}\right)-\frac{2}{\alpha} \omega_{J}\left(\mu, \mu_{J}^{(L)}\right)\right) \Gamma\left(-\frac{2(\beta+1)}{\alpha+\beta} \omega_{S_{C}}\left(\mu, \mu_{S_{C}}^{(R)}\right)-\frac{2}{\alpha} \omega_{J}\left(\mu, \mu_{J}^{(R)}\right)\right)\right]^{-1} .
\end{aligned}
$$

\section{G Renormalization group evolution of $D_{k}$}

In this appendix we discuss in detail the renormalization group evolution of the jet flavor coefficient $D_{k}$ and explain the procedure we used to estimate the scale uncertainty introduced by neglecting higher-order terms.

The cross section for soft-drop groomed jets in $p p \rightarrow Z+j$ events factorizes in the limit $e_{2}^{(\alpha)} \ll z_{\text {cut }} \ll 1$, where

$$
\frac{d \sigma_{\mathrm{resum}}}{d e_{2}^{(\alpha)}}=\sum_{k=q, \bar{q}, g} D_{k}\left(p_{T}^{\min }, \eta_{\max }, z_{\mathrm{cut}}, R\right) S_{C, k}\left(z_{\mathrm{cut}} e_{2}^{(\alpha)}\right) \otimes J_{k}\left(e_{2}^{(\alpha)}\right) .
$$

The fact that $D_{k}$ depends on multiple scales prohibits its resummation to all orders. Nevertheless, its renormalization scale dependence is completely determined by renormalization group invariance of the cross section. We can improve our prediction by solving the following renormalization group equation, which holds at leading power in $z_{\text {cut }}$ :

$$
\begin{aligned}
\frac{\partial \log D_{k}}{\partial \log \mu} & =-\frac{\partial \log \left(J_{k} \otimes S_{C, k}\right)}{\partial \log \mu} \\
& =\Gamma_{D_{k}}\left(\alpha_{s}\right) \log \left(\frac{\mu^{2}}{Q^{2}}\right)+\gamma_{D_{k}}\left(\alpha_{s}, z_{\text {cut }}\right),
\end{aligned}
$$

where $Q=2 \bar{p}_{T J}$. The anomalous dimensions $\Gamma_{D_{k}}$ and $\gamma_{D_{k}}$ are

$$
\begin{aligned}
& \Gamma_{D_{k}}=-\frac{\beta}{1+\beta} C_{k} \Gamma_{\text {cusp }}, \\
& \gamma_{D_{k}}=-\left(\gamma_{J_{k}}+\gamma_{S_{C, k}}\right)-\frac{C_{k}}{1+\beta} \Gamma_{\text {cusp }} \log z_{\text {cut }}^{2} .
\end{aligned}
$$

Here, $C_{k}$ is the color Casimir for the jet of flavor $k$. The anomalous dimension has $\log z_{\text {cut }}$ dependence, which means $Q$ is not a natural scale of $D_{k}$ where all logarithms are minimized.

Nevertheless, we can still formally evolve $D_{k}$ from a scale $\mu_{0} \sim Q$ to a renormalization scale $\mu$ common to the jet and collinear-soft function. Solving the renormalization group evolution eq. (G.2), the improved $D_{k}$ takes the form

$$
D_{k}\left(\mu, \mu_{f}\right) \equiv D_{k}\left(\alpha_{s}, \mu_{0}, \mu_{f}\right)\left(\frac{\mu_{0}^{2}}{Q^{2}}\right)^{\omega_{D_{k}}\left(\mu, \mu_{0}\right)} e^{K_{D_{k}}\left(\mu, \mu_{0}\right)} .
$$


Here, $\mu_{f}$ represents the factorization scale; i.e., the scale at which the parton distribution functions in $D_{k}$ are defined. The $\omega_{D_{k}}$ and $K_{D_{k}}$ functions are defined in appendix F. To estimate uncertainties from higher-order corrections due to residual scale dependence in $D_{k}$, we will vary both $\mu_{0}$ and $\mu_{f}$ over the values

$$
\begin{aligned}
& \mu_{0}=\left\{\frac{Q}{2}, Q, 2 Q\right\}, \\
& \mu_{f}=\left\{\frac{Q}{2}, Q, 2 Q\right\} .
\end{aligned}
$$

For evaluating $D_{k}$ at fixed-order, we keep the full leading and next-to-leading terms as well as singular terms at the next-to-next-to-leading order in the following expansion of the solution to the renormalization group equation, eq. (G.5). Expanding $D_{k}$ in powers of $\alpha_{s}$ as

$$
D_{k}\left(\alpha_{s}, \mu, \mu_{f}\right)=\sum_{n=0}\left(\frac{\alpha_{s}(\mu)}{4 \pi}\right)^{n} D_{k}^{(n)}\left(\mu, \mu_{f}\right),
$$

we have the solutions:

$$
\begin{aligned}
D_{k}^{(0)}= & c_{D_{k}}^{(0)}, \\
D_{k}^{(1)}= & \Gamma_{D_{k}}^{(0)} c_{D_{k}}^{(0)} \log ^{2} \frac{\mu}{Q}+\left(\gamma_{D_{k}}^{(0)} c_{D_{k}}^{(0)}+2 \beta_{0} c_{D_{k}}^{(0)}\right) \log \frac{\mu}{Q}+c_{D_{k}}^{(1)}, \\
D_{k}^{(2)}= & \frac{1}{2}\left(\Gamma_{D_{k}}^{(0)}\right)^{2} c_{D_{k}}^{(0)} \log ^{4} \frac{\mu}{\mu_{0}}+\left(\gamma_{D_{k}}^{(0)} \Gamma_{D_{k}}^{(0)}+\frac{8}{3} \beta_{0} \Gamma_{D_{k}}^{(0)}\right) c_{D_{k}}^{(0)} \log ^{3} \frac{\mu}{Q} \\
& +\left[\left(\Gamma_{D_{k}}^{(1)}+\frac{1}{2}\left(\gamma_{D_{k}}^{(0)}\right)^{2}+3 \beta_{0} \gamma_{D_{k}}^{(0)}\right) c_{D_{k}}^{(0)}+\Gamma_{D_{k}}^{(0)} c_{D_{k}}^{(1)}\right] \log ^{2} \frac{\mu}{Q} \\
& +\left[\left(\gamma_{D_{k}}^{(1)}+2 \beta_{1}\right) c_{D_{k}}^{(0)}+\left(\gamma_{D_{k}}^{(0)}+4 \beta_{0}\right) c_{D_{k}}^{(1)}\right] \log \frac{\mu}{Q}+c_{D_{k}}^{(2)} .
\end{aligned}
$$

The non-singular terms $c_{D_{k}}^{(n)}$ are defined such that at $\mu=Q$,

$$
D_{k}^{(n)}\left(Q, \mu_{f}\right)=c_{D_{k}}^{(n)}\left(Q, \mu_{f}\right)
$$

Therefore one can extract the value of $c_{D_{k}}^{(0)}\left(Q, \mu_{f}\right)$ and $c_{D_{k}}^{(1)}\left(Q, \mu_{f}\right)$ from MCFM and then extrapolate $D_{k}$ to arbitrary value of $\mu_{0}$. Given the current level of precision of MCFM, this procedure can be done through the next-to-leading order. At $\mathcal{O}\left(\alpha_{s}^{3}\right)$, the $c_{D_{k}}^{(2)}$ term cannot be determined without the next-to-next-to-leading $p p \rightarrow Z+j$ cross section. Note that the size of $c_{D_{k}}^{(2)}$ is no greater than $\mathcal{O}\left(\alpha_{s}^{3} \log ^{4} z_{\text {cut }}\right)$. Thus we can estimate that the size of uncertainty introduced by the unknown higher-loop non-singular term $c_{D_{k}}^{(2)}$ is roughly a factor of $1 \pm \alpha_{s}^{2} \log ^{4} z_{\text {cut }} e^{\alpha_{s}^{n} L^{n+1}+\cdots}$, which is beyond NNLL accuracy.

Open Access. This article is distributed under the terms of the Creative Commons Attribution License (CC-BY 4.0), which permits any use, distribution and reproduction in any medium, provided the original author(s) and source are credited. 


\section{References}

[1] M. Cacciari, G.P. Salam and G. Soyez, The catchment area of jets, JHEP 04 (2008) 005 [arXiv:0802.1188] [INSPIRE].

[2] J.M. Butterworth, A.R. Davison, M. Rubin and G.P. Salam, Jet substructure as a new Higgs search channel at the LHC, Phys. Rev. Lett. 100 (2008) 242001 [arXiv:0802.2470] [INSPIRE].

[3] S.D. Ellis, C.K. Vermilion and J.R. Walsh, Recombination algorithms and jet substructure: pruning as a tool for heavy particle searches, Phys. Rev. D 81 (2010) 094023 [arXiv:0912.0033] [INSPIRE].

[4] D. Krohn, J. Thaler and L.-T. Wang, Jet trimming, JHEP 02 (2010) 084 [arXiv:0912.1342] [INSPIRE].

[5] G. Soyez, G.P. Salam, J. Kim, S. Dutta and M. Cacciari, Pileup subtraction for jet shapes, Phys. Rev. Lett. 110 (2013) 162001 [arXiv:1211.2811] [INSPIRE].

[6] M. Dasgupta, A. Fregoso, S. Marzani and G.P. Salam, Towards an understanding of jet substructure, JHEP 09 (2013) 029 [arXiv: 1307.0007] [INSPIRE].

[7] D. Krohn, M.D. Schwartz, M. Low and L.-T. Wang, Jet cleansing: pileup removal at high luminosity, Phys. Rev. D 90 (2014) 065020 [arXiv: 1309.4777] [INSPIRE].

[8] A.J. Larkoski, S. Marzani, G. Soyez and J. Thaler, Soft drop, JHEP 05 (2014) 146 [arXiv: 1402 .2657] [INSPIRE].

[9] P. Berta, M. Spousta, D.W. Miller and R. Leitner, Particle-level pileup subtraction for jets and jet shapes, JHEP 06 (2014) 092 [arXiv:1403.3108] [INSPIRE].

[10] M. Cacciari, G.P. Salam and G. Soyez, SoftKiller, a particle-level pileup removal method, Eur. Phys. J. C 75 (2015) 59 [arXiv:1407.0408] [InSPIRE].

[11] D. Bertolini, P. Harris, M. Low and N. Tran, Pileup per particle identification, JHEP 10 (2014) 059 [arXiv:1407.6013] [INSPIRE].

[12] M. Dasgupta, A. Fregoso, S. Marzani and A. Powling, Jet substructure with analytical methods, Eur. Phys. J. C 73 (2013) 2623 [arXiv:1307.0013] [InSPIRE].

[13] M. Dasgupta, A. Powling and A. Siodmok, On jet substructure methods for signal jets, JHEP 08 (2015) 079 [arXiv: 1503.01088] [INSPIRE].

[14] M. Dasgupta and G.P. Salam, Resummation of nonglobal QCD observables, Phys. Lett. B 512 (2001) 323 [hep-ph/0104277] [INSPIRE].

[15] C.W. Bauer, S. Fleming, D. Pirjol and I.W. Stewart, An effective field theory for collinear and soft gluons: heavy to light decays, Phys. Rev. D 63 (2001) 114020 [hep-ph/0011336] [INSPIRE].

[16] C.W. Bauer and I.W. Stewart, Invariant operators in collinear effective theory, Phys. Lett. B 516 (2001) 134 [hep-ph/0107001] [INSPIRE].

[17] C.W. Bauer, D. Pirjol and I.W. Stewart, Soft collinear factorization in effective field theory, Phys. Rev. D 65 (2002) 054022 [hep-ph/0109045] [InSPIRE].

[18] C.W. Bauer, S. Fleming, D. Pirjol, I.Z. Rothstein and I.W. Stewart, Hard scattering factorization from effective field theory, Phys. Rev. D 66 (2002) 014017 [hep-ph/0202088] [INSPIRE]. 
[19] C. Frye, A.J. Larkoski, M.D. Schwartz and K. Yan, Precision physics with pile-up insensitive observables, arXiv:1603.06375 [INSPIRE].

[20] A. Banfi, G.P. Salam and G. Zanderighi, Principles of general final-state resummation and automated implementation, JHEP 03 (2005) 073 [hep-ph/0407286] [INSPIRE].

[21] M. Jankowiak and A.J. Larkoski, Jet substructure without trees, JHEP 06 (2011) 057 [arXiv: 1104.1646] [INSPIRE].

[22] A.J. Larkoski, G.P. Salam and J. Thaler, Energy correlation functions for jet substructure, JHEP 06 (2013) 108 [arXiv:1305.0007] [InSPIRE].

[23] C.W. Bauer, F.J. Tackmann, J.R. Walsh and S. Zuberi, Factorization and resummation for dijet invariant mass spectra, Phys. Rev. D 85 (2012) 074006 [arXiv:1106.6047] [InSPIRE].

[24] M. Procura, W.J. Waalewijn and L. Zeune, Resummation of double-differential cross sections and fully-unintegrated parton distribution functions, JHEP 02 (2015) 117 [arXiv: 1410.6483] [INSPIRE].

[25] A.J. Larkoski, I. Moult and D. Neill, Non-global logarithms, factorization and the soft substructure of jets, JHEP 09 (2015) 143 [arXiv:1501.04596] [INSPIRE].

[26] A.J. Larkoski, I. Moult and D. Neill, Analytic boosted boson discrimination, JHEP 05 (2016) 117 [arXiv: 1507.03018] [InSPIRE].

[27] T. Becher, M. Neubert, L. Rothen and D.Y. Shao, Effective field theory for jet processes, Phys. Rev. Lett. 116 (2016) 192001 [arXiv:1508.06645] [INSPIRE].

[28] Y.-T. Chien, A. Hornig and C. Lee, Soft-collinear mode for jet cross sections in soft collinear effective theory, Phys. Rev. D 93 (2016) 014033 [arXiv:1509.04287] [INSPIRE].

[29] A. von Manteuffel, R.M. Schabinger and H.X. Zhu, The complete two-loop integrated jet thrust distribution in soft-collinear effective theory, JHEP 03 (2014) 139 [arXiv:1309.3560] [INSPIRE].

[30] A. Banfi, G.P. Salam and G. Zanderighi, $N L L+N N L O$ predictions for jet-veto efficiencies in Higgs-boson and Drell-Yan production, JHEP 06 (2012) 159 [arXiv:1203.5773] [INSPIRE].

[31] T. Becher and M. Neubert, Factorization and NNLL resummation for Higgs production with a jet veto, JHEP 07 (2012) 108 [arXiv:1205.3806] [INSPIRE].

[32] A. Banfi, P.F. Monni, G.P. Salam and G. Zanderighi, Higgs and Z-boson production with a jet veto, Phys. Rev. Lett. 109 (2012) 202001 [arXiv:1206.4998] [INSPIRE].

[33] T. Becher, M. Neubert and L. Rothen, Factorization and $N^{3} L L_{p}+N N L O$ predictions for the Higgs cross section with a jet veto, JHEP 10 (2013) 125 [arXiv:1307.0025] [INSPIRE].

[34] I.W. Stewart, F.J. Tackmann, J.R. Walsh and S. Zuberi, Jet $p_{T}$ resummation in Higgs production at $N N L L^{\prime}+N N L O$, Phys. Rev. D 89 (2014) 054001 [arXiv:1307.1808] [INSPIRE].

[35] S. Catani and M.H. Seymour, A general algorithm for calculating jet cross-sections in NLO QCD, Nucl. Phys. B 485 (1997) 291 [Erratum ibid. B 510 (1998) 503] [hep-ph/9605323] [INSPIRE].

[36] G. Bell, R. Rahn and J. Talbert, Automated calculation of dijet soft functions in soft-collinear effective theory, in Proceedings, $12^{\text {th }}$ International Symposium on Radiative Corrections (Radcor 2015) and LoopFest XIV (Radiative Corrections for the LHC and Future Colliders), (2015) [arXiv: 1512.06100] [INSPIRE]. 
[37] C.F. Berger, T. Kucs and G.F. Sterman, Event shape/energy flow correlations, Phys. Rev. D 68 (2003) 014012 [hep-ph/0303051] [INSPIRE].

[38] L.G. Almeida, S.J. Lee, G. Perez, G.F. Sterman, I. Sung and J. Virzi, Substructure of high-p ${ }_{T}$ jets at the LHC, Phys. Rev. D 79 (2009) 074017 [arXiv:0807.0234] [InSPIRE].

[39] S.D. Ellis, C.K. Vermilion, J.R. Walsh, A. Hornig and C. Lee, Jet shapes and jet algorithms in SCET, JHEP 11 (2010) 101 [arXiv:1001.0014] [INSPIRE].

[40] A.J. Larkoski, D. Neill and J. Thaler, Jet shapes with the broadening axis, JHEP 04 (2014) 017 [arXiv: 1401.2158] [INSPIRE].

[41] Y.-T. Chien, R. Kelley, M.D. Schwartz and H.X. Zhu, Resummation of jet mass at hadron colliders, Phys. Rev. D 87 (2013) 014010 [arXiv:1208.0010] [INSPIRE].

[42] T.T. Jouttenus, I.W. Stewart, F.J. Tackmann and W.J. Waalewijn, Jet mass spectra in Higgs boson plus one jet at next-to-next-to-leading logarithmic order, Phys. Rev. D 88 (2013) 054031 [arXiv: 1302.0846] [INSPIRE].

[43] M. Dasgupta, K. Khelifa-Kerfa, S. Marzani and M. Spannowsky, On jet mass distributions in $Z+$ jet and dijet processes at the LHC, JHEP 10 (2012) 126 [arXiv:1207.1640] [INSPIRE].

[44] I. Feige, M.D. Schwartz, I.W. Stewart and J. Thaler, Precision jet substructure from boosted event shapes, Phys. Rev. Lett. 109 (2012) 092001 [arXiv:1204.3898] [INSPIRE].

[45] S. Catani, Y.L. Dokshitzer, M. Olsson, G. Turnock and B.R. Webber, New clustering algorithm for multi-jet cross-sections in $e^{+} e^{-}$annihilation, Phys. Lett. B 269 (1991) 432 [INSPIRE].

[46] S. Catani, Y.L. Dokshitzer, M.H. Seymour and B.R. Webber, Longitudinally invariant $K_{t}$ clustering algorithms for hadron hadron collisions, Nucl. Phys. B 406 (1993) 187 [INSPIRE].

[47] S.D. Ellis and D.E. Soper, Successive combination jet algorithm for hadron collisions, Phys. Rev. D 48 (1993) 3160 [hep-ph/9305266] [INSPIRE].

[48] Y.L. Dokshitzer, G.D. Leder, S. Moretti and B.R. Webber, Better jet clustering algorithms, JHEP 08 (1997) 001 [hep-ph/9707323] [INSPIRE].

[49] M. Wobisch and T. Wengler, Hadronization corrections to jet cross-sections in deep inelastic scattering, in Monte Carlo generators for HERA physics. Proceedings, Workshop, Hamburg Germany (1998)-(1999) [hep-ph/9907280] [INSPIRE].

[50] M. Wobisch, Measurement and QCD analysis of jet cross-sections in deep inelastic positron proton collisions at $\sqrt{s}=300 \mathrm{GeV}$, Ph.D. thesis, Tech. Hochsch., Aachen Germany (2000).

[51] M. Cacciari, G.P. Salam and G. Soyez, The anti- $k_{t}$ jet clustering algorithm, JHEP 04 (2008) 063 [arXiv:0802.1189] [INSPIRE].

[52] S. Fleming, A.H. Hoang, S. Mantry and I.W. Stewart, Jets from massive unstable particles: top-mass determination, Phys. Rev. D 77 (2008) 074010 [hep-ph/0703207] [INSPIRE].

[53] M.D. Schwartz, Resummation and NLO matching of event shapes with effective field theory, Phys. Rev. D 77 (2008) 014026 [arXiv:0709.2709] [INSPIRE].

[54] M. Beneke and V.A. Smirnov, Asymptotic expansion of Feynman integrals near threshold, Nucl. Phys. B 522 (1998) 321 [hep-ph/9711391] [INSPIRE].

[55] T. Becher, A. Broggio and A. Ferroglia, Introduction to soft-collinear effective theory, Lect. Notes Phys. 896 (2015) 1 [arXiv:1410.1892] [InSPIRE].

[56] I. Feige and M.D. Schwartz, An on-shell approach to factorization, Phys. Rev. D 88 (2013) 065021 [arXiv: 1306.6341] [INSPIRE]. 
[57] I. Feige and M.D. Schwartz, Hard-soft-collinear factorization to all orders, Phys. Rev. D 90 (2014) 105020 [arXiv:1403.6472] [INSPIRE].

[58] I. Feige, M.D. Schwartz and K. Yan, Removing phase-space restrictions in factorized cross sections, Phys. Rev. D 91 (2015) 094027 [arXiv: 1502.05411] [INSPIRE].

[59] A. Hornig, C. Lee, I.W. Stewart, J.R. Walsh and S. Zuberi, Non-global structure of the $O\left(\alpha_{s}^{2}\right)$ dijet soft function, JHEP 08 (2011) 054 [arXiv: 1105.4628] [INSPIRE].

[60] A. Hornig, C. Lee, J.R. Walsh and S. Zuberi, Double non-global logarithms in-n-out of jets, JHEP 01 (2012) 149 [arXiv:1110.0004] [INSPIRE].

[61] R. Kelley, M.D. Schwartz, R.M. Schabinger and H.X. Zhu, The two-loop hemisphere soft function, Phys. Rev. D 84 (2011) 045022 [arXiv:1105.3676] [InSPIRE].

[62] M.D. Schwartz and H.X. Zhu, Nonglobal logarithms at three loops, four loops, five loops and beyond, Phys. Rev. D 90 (2014) 065004 [arXiv:1403.4949] [INSPIRE].

[63] K. Khelifa-Kerfa and Y. Delenda, Non-global logarithms at finite $N_{c}$ beyond leading order, JHEP 03 (2015) 094 [arXiv: 1501.00475] [INSPIRE].

[64] S. Caron-Huot, Resummation of non-global logarithms and the BFKL equation, arXiv: 1501.03754 [INSPIRE].

[65] D. Neill, The edge of jets and subleading non-global logs, arXiv:1508.07568 [INSPIRE].

[66] L.G. Almeida, S.D. Ellis, C. Lee, G. Sterman, I. Sung and J.R. Walsh, Comparing and counting logs in direct and effective methods of QCD resummation, JHEP 04 (2014) 174 [arXiv: 1401.4460] [INSPIRE].

[67] G.P. Korchemsky and A.V. Radyushkin, Renormalization of the Wilson loops beyond the leading order, Nucl. Phys. B 283 (1987) 342 [INSPIRE].

[68] A. Vogt, Next-to-next-to-leading logarithmic threshold resummation for deep inelastic scattering and the Drell-Yan process, Phys. Lett. B 497 (2001) 228 [hep-ph/0010146] [INSPIRE].

[69] C.F. Berger, Higher orders in $A\left(\alpha_{s}\right) /[1-x]_{+}$of nonsinglet partonic splitting functions, Phys. Rev. D 66 (2002) 116002 [hep-ph/0209107] [INSPIRE].

[70] S. Moch, J.A.M. Vermaseren and A. Vogt, Three-loop results for quark and gluon form-factors, Phys. Lett. B 625 (2005) 245 [hep-ph/0508055] [INSPIRE].

[71] O.V. Tarasov, A.A. Vladimirov and A. Yu. Zharkov, The Gell-Mann-Low function of QCD in the three loop approximation, Phys. Lett. B 93 (1980) 429 [INSPIRE].

[72] S.A. Larin and J.A.M. Vermaseren, The three loop QCD $\beta$-function and anomalous dimensions, Phys. Lett. B 303 (1993) 334 [hep-ph/9302208] [INSPIRE].

[73] W.L. van Neerven, Dimensional regularization of mass and infrared singularities in two loop on-shell vertex functions, Nucl. Phys. B 268 (1986) 453 [INSPIRE].

[74] T. Matsuura, S.C. van der Marck and W.L. van Neerven, The calculation of the second order soft and virtual contributions to the Drell-Yan cross-section, Nucl. Phys. B 319 (1989) 570 [INSPIRE].

[75] C.W. Bauer and A.V. Manohar, Shape function effects in $B \rightarrow X_{s} \gamma$ and $B \rightarrow X_{u} \ell \bar{\nu}$ decays, Phys. Rev. D 70 (2004) 034024 [hep-ph/0312109] [InSPIRE].

[76] S.W. Bosch, B.O. Lange, M. Neubert and G. Paz, Factorization and shape function effects in inclusive B meson decays, Nucl. Phys. B 699 (2004) 335 [hep-ph/0402094] [INSPIRE]. 
[77] T. Becher and G. Bell, The gluon jet function at two-loop order, Phys. Lett. B 695 (2011) 252 [arXiv: 1008.1936] [INSPIRE].

[78] T. Becher and M. Neubert, Toward a NNLO calculation of the $\bar{B} \rightarrow X_{s} \gamma$ decay rate with a cut on photon energy. II. Two-loop result for the jet function, Phys. Lett. B 637 (2006) 251 [hep-ph/0603140] [INSPIRE].

[79] M. Neubert, Renormalization-group improved calculation of the $B \rightarrow X_{s} \gamma$ branching ratio, Eur. Phys. J. C 40 (2005) 165 [hep-ph/0408179] [INSPIRE].

[80] T. Becher, M. Neubert and B.D. Pecjak, Factorization and momentum-space resummation in deep-inelastic scattering, JHEP 01 (2007) 076 [hep-ph/0607228] [INSPIRE].

[81] S. Catani and M. Grazzini, Infrared factorization of tree level QCD amplitudes at the next-to-next-to-leading order and beyond, Nucl. Phys. B 570 (2000) 287 [hep-ph/9908523] [INSPIRE].

[82] Y.-T. Chien and M.D. Schwartz, Resummation of heavy jet mass and comparison to LEP data, JHEP 08 (2010) 058 [arXiv: 1005.1644] [INSPIRE].

[83] M. Bahr et al., HERWIG++ physics and manual, Eur. Phys. J. C 58 (2008) 639 [arXiv:0803.0883] [INSPIRE].

[84] J. Bellm et al., HERWIG++ 2.7 release note, arXiv:1310.6877 [INSPIRE].

[85] T. Sjöstrand, S. Mrenna and P.Z. Skands, PYTHIA 6.4 physics and manual, JHEP 05 (2006) 026 [hep-ph/0603175] [INSPIRE].

[86] T. Sjöstrand et al., An introduction to PYTHIA 8.2, Comput. Phys. Commun. 191 (2015) 159 [arXiv:1410.3012] [INSPIRE].

[87] W.T. Giele, D.A. Kosower and P.Z. Skands, A simple shower and matching algorithm, Phys. Rev. D 78 (2008) 014026 [arXiv:0707.3652] [INSPIRE].

[88] W.T. Giele, D.A. Kosower and P.Z. Skands, Higher-order corrections to timelike jets, Phys. Rev. D 84 (2011) 054003 [arXiv: 1102.2126] [InSPIRE].

[89] L. Hartgring, E. Laenen and P. Skands, Antenna showers with one-loop matrix elements, JHEP 10 (2013) 127 [arXiv:1303.4974] [INSPIRE].

[90] A.J. Larkoski, J.J. Lopez-Villarejo and P. Skands, Helicity-dependent showers and matching with VINCIA, Phys. Rev. D 87 (2013) 054033 [arXiv: 1301.0933] [INSPIRE].

[91] S. Catani, B.R. Webber and G. Marchesini, QCD coherent branching and semiinclusive processes at large $x$, Nucl. Phys. B 349 (1991) 635 [InSPIRE].

[92] Y.L. Dokshitzer, V.A. Khoze and S.I. Troian, Specific features of heavy quark production. LPHD approach to heavy particle spectra, Phys. Rev. D 53 (1996) 89 [hep-ph/9506425] [INSPIRE].

[93] M. Cacciari, G.P. Salam and G. Soyez, FastJet user manual, Eur. Phys. J. C 72 (2012) 1896 [arXiv: 1111.6097] [INSPIRE].

[94] G.P. Korchemsky and G.F. Sterman, Power corrections to event shapes and factorization, Nucl. Phys. B 555 (1999) 335 [hep-ph/9902341] [INSPIRE].

[95] G.P. Korchemsky and S. Tafat, On power corrections to the event shape distributions in QCD, JHEP 10 (2000) 010 [hep-ph/0007005] [INSPIRE].

[96] I.W. Stewart, F.J. Tackmann and W.J. Waalewijn, Dissecting soft radiation with factorization, Phys. Rev. Lett. 114 (2015) 092001 [arXiv:1405.6722] [INSPIRE]. 
[97] T. Becher, R. Frederix, M. Neubert and L. Rothen, Automated NNLL $+N L O$ resummation for jet-veto cross sections, Eur. Phys. J. C 75 (2015) 154 [arXiv: 1412.8408] [InSPIRE].

[98] D. Farhi, I. Feige, M. Freytsis and M.D. Schwartz, Streamlining resummed QCD calculations using Monte Carlo integration, arXiv:1507.06315 [INSPIRE].

[99] A. Banfi, G.P. Salam and G. Zanderighi, Infrared safe definition of jet flavor, Eur. Phys. J. C 47 (2006) 113 [hep-ph/0601139] [INSPIRE].

[100] J.M. Campbell and R.K. Ellis, Next-to-leading order corrections to $W+2$ jet and $Z+2$ jet production at hadron colliders, Phys. Rev. D 65 (2002) 113007 [hep-ph/0202176] [INSPIRE].

[101] J.M. Campbell, R.K. Ellis and D.L. Rainwater, Next-to-leading order QCD predictions for $W+2$ jet and $Z+2$ jet production at the CERN LHC, Phys. Rev. D 68 (2003) 094021 [hep-ph/0308195] [INSPIRE].

[102] A.D. Martin, W.J. Stirling, R.S. Thorne and G. Watt, Parton distributions for the LHC, Eur. Phys. J. C 63 (2009) 189 [arXiv:0901.0002] [InSPIRE].

[103] Fastjet contrib webpage, http://fastjet.hepforge.org/contrib/.

[104] J.C. Collins, D.E. Soper and G.F. Sterman, Transverse momentum distribution in Drell-Yan pair and $W$ and $Z$ boson production, Nucl. Phys. B 250 (1985) 199 [INSPIRE].

[105] I.Z. Rothstein and I.W. Stewart, An effective field theory for forward scattering and factorization violation, arXiv:1601.04695 [INSPIRE].

[106] T. Becher and M.D. Schwartz, A precise determination of $\alpha_{s}$ from LEP thrust data using effective field theory, JHEP 07 (2008) 034 [arXiv:0803.0342] [INSPIRE].

[107] R. Abbate, M. Fickinger, A.H. Hoang, V. Mateu and I.W. Stewart, Thrust at $N^{3} L L$ with power corrections and a precision global fit for $\alpha_{s}\left(m_{Z}\right)$, Phys. Rev. D 83 (2011) 074021 [arXiv: 1006 .3080] [INSPIRE].

[108] A.H. Hoang, D.W. Kolodrubetz, V. Mateu and I.W. Stewart, C-parameter distribution at $N^{3} L L$ ' including power corrections, Phys. Rev. D 91 (2015) 094017 [arXiv:1411.6633] [INSPIRE].

[109] A. Gehrmann-De Ridder, T. Gehrmann, E.W.N. Glover and G. Heinrich, EERAD3: event shapes and jet rates in electron-positron annihilation at order $\alpha_{s}^{3}$, Comput. Phys. Commun. 185 (2014) 3331 [arXiv: 1402.4140] [INSPIRE].

[110] C.W. Bauer, C. Lee, A.V. Manohar and M.B. Wise, Enhanced nonperturbative effects in Z decays to hadrons, Phys. Rev. D 70 (2004) 034014 [hep-ph/0309278] [INSPIRE].

[111] A.V. Manohar, Deep inelastic scattering as $x \rightarrow 1$ using soft collinear effective theory, Phys. Rev. D 68 (2003) 114019 [hep-ph/0309176] [INSPIRE].

[112] R.V. Harlander, Virtual corrections to $g g \rightarrow H$ to two loops in the heavy top limit, Phys. Lett. B 492 (2000) 74 [hep-ph/0007289] [INSPIRE].

[113] C. Anastasiou and K. Melnikov, Pseudoscalar Higgs boson production at hadron colliders in NNLO QCD, Phys. Rev. D 67 (2003) 037501 [hep-ph/0208115] [INSPIRE].

[114] V. Ravindran, J. Smith and W.L. van Neerven, Two-loop corrections to Higgs boson production, Nucl. Phys. B 704 (2005) 332 [hep-ph/0408315] [INSPIRE].

[115] T. Becher and M.D. Schwartz, Direct photon production with effective field theory, JHEP 02 (2010) 040 [arXiv:0911.0681] [INSPIRE]. 